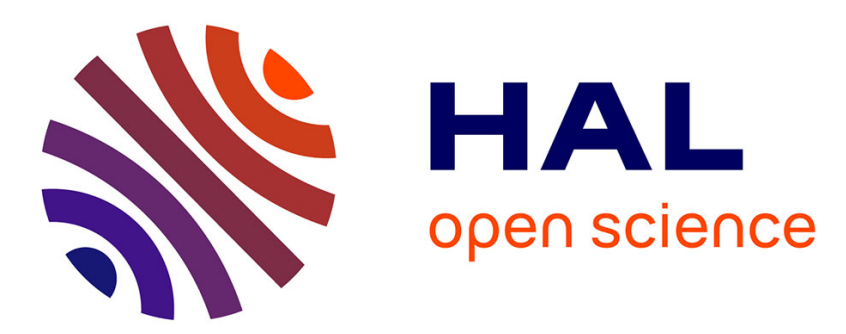

\title{
Raman Spectroscopy of Nanomaterials: How Spectra Relate to Disorder, Particle Size and Mechanical Properties
}

Gwenaël Gouadec, Philippe Colomban

\section{- To cite this version:}

Gwenaël Gouadec, Philippe Colomban. Raman Spectroscopy of Nanomaterials: How Spectra Relate to Disorder, Particle Size and Mechanical Properties. Progress in Crystal Growth and Characterization of Materials, 2007, 53 (1), pp.1-56. 10.1016/j.pcrysgrow.2007.01.001 · hal-00120432

\section{HAL Id: hal-00120432 \\ https://hal.science/hal-00120432}

Submitted on 14 Dec 2006

HAL is a multi-disciplinary open access archive for the deposit and dissemination of scientific research documents, whether they are published or not. The documents may come from teaching and research institutions in France or abroad, or from public or private research centers.
L'archive ouverte pluridisciplinaire HAL, est destinée au dépôt et à la diffusion de documents scientifiques de niveau recherche, publiés ou non, émanant des établissements d'enseignement et de recherche français ou étrangers, des laboratoires publics ou privés. 


\section{Raman Spectroscopy of Nanomaterials: How Spectra Relate to Disorder, Particle Size and Mechanical Properties}

\section{Gwénaël GOUADEC and Philippe COLOMBAN}

Laboratoire de Dynamique, Interactions et Réactivité (LADIR), UMR7075 - CNRS \& Université Pierre et Marie Curie - Paris 6, 2 rue Henry Dunant, 94320 Thiais, France

\section{ABSTRACT}

The purpose of this review is to provide non-specialists with a basic understanding of the information micro-Raman Spectroscopy ( $\mu \mathrm{RS})$ may yield when this characterization tool is applied to nanomaterials, a generic term for describing nano-sized crystals and bulk homogeneous materials with a structural disorder at the nanoscale - typically nanoceramics, nanocomposites, glassy materials and relaxor ferroelectrics. The selected materials include advanced and ancient ceramics, semiconductors and polymers developed in the form of dots, wires, films, fibres or composites for applications in the energy, electronic and aeronauticsaerospace industries. Following a short introduction, the text is divided into four sections:

- The $1^{\text {st }}$ section outlines the principles of conventional $\mu \mathrm{RS}$.

- The $2^{\text {nd }}$ section introduces the main effects for nanomaterials, with special emphasis on two models that connect Raman spectra features to "grain size", namely the Phonon Confinement Model (PCM) and the Elastic Sphere Model (ESM).

- The $3^{\text {rd }}$ section presents the experimental versatility of $\mu$ RS applied to nanomaterials (phase identification, phase transition monitoring, grain size determination, defect concentration assessment, etc.).

- The $4^{\text {th }}$ section deals with the micro-mechanical aspects of $\mu$ RS ("Raman extensometry"). Special emphasis is placed on the relationship between the stress-related coefficients $S^{\varepsilon / \sigma}$ and the macroscopic response of the materials to the applied stress.

PACS: 63.20.-e; 63.50.+x; 78.30.-j; 81.40.Jj; 81.05.Ys

Keywords: A1 Raman Spectroscopy; A1 disorder; A1 stress; B1 nanomaterials; B1 nanotubes; B1 oxides; B2 Semiconductors; B2 carbon; B2 glass 


\section{INTRODUCTION}

Most properties of traditional ceramics (notably a good shapability and low sintering temperatures) stem from the fact that their raw material -natural clay- is nanosized [1]. Besides, because of the sharpness of the human eye, the particle size of pigments must be smaller than $500 \mathrm{~nm}$ to homogeneously colour enamels and glasses [2]. Potters and ceramists have thus been using nanoscience for thousands of years [3,4] but a new generation of engineered nanomaterials (grain size $<100 \mathrm{~nm}$ ) has been in development -if not already commercially available- for the last twenty years. Reducing the dimension of matter domains down to the nanometer scale confines the electronic and vibrational wavefunctions while increasing the specific surface, which results in unique properties and opens a wide range of potential applications in domains as different as [5-8]:

Optics: pigments for the cosmetic industry (metal-oxides), fluorescent markers (quantum dots), photonic crystals (multiplexing and switching in optical networks), quantum computer components, light emitting devices [9], etc.

Mechanics: cutting tools, wear-resistant and anti-corrosion coatings (cemented carbides), "nano-polishing" powders ( $\mathrm{SiC}$, diamond, boron carbide), fibres and fibre-reinforced composites, structural nanocomposites [10], etc.

Electrical devices: miniaturized silicon chips, single electron transistors, relaxor ferroelectrics [11-14], carbon or silicon nanotube transistors, lithium batteries [15], solar cells [16], etc.

Magnetic devices: data storage, giant magneto-resistances (reading heads), etc.

Reactivity: improved combustion of fuel-rich propellants (Al, Ti, Ni, B) [17], filters (Ti/Zr oxides), nanosensors [18,19], catalysts [20], etc.

Biomedicine: in vivo drug delivery, diagnostic and monitoring devices, [21] fluorescent markers, etc.

The challenge for the so-called nanotechnologies is to achieve perfect control of nanoscalerelated properties. This obviously requires correlating the parameters of the synthesis process (self assembly, microlithography, sol-gel, polymer curing, electrochemical deposition, laser ablation...) with the resulting nanostructure. Not all conventional techniques are suitable for that purpose but Raman Spectroscopy (RS) has already proven to be. For quite a long time this technique was mainly devoted to fundamental research, but instrumental progress (laser miniaturization, CCD detection, notch filters and data processing softwares) have rendered it a general characterization method. Not only can it provide basic phase identification but subtle spectra alterations can be used to assess nano-scale structural changes and characterize micromechanical behaviour. RS thus is a unique tool for probing or mapping nanophases dispersed in a matrix (e.g. pigments in a ceramic glaze [2], precipitates in a fibre coating [22]), surface-formed nanophases (corrosion mechanisms [23]) and solid-state devices [2427]. Some specific features can even be used to study a charge transfer [28,29], a film orientation [30], the size of clusters trapped in nano-cavities [31], Grüneisen parameter [32], configurational order (for instance the proportion of trans-gauche chains in Poly(ethylene terephtalate)-PET [33]) or intercalation [34], interfacial [35] and polymerisation [36] reactions.

The present article, which is an extended version of previous papers from our group [37-39], is intended to review the achievements of RS in the world of nanomaterials, both from the fundamental and experimental points of view. The selected materials include advanced and 
ancient ceramics, glasses, semiconductors and polymers developed in the form of dots, wires, films, fibres or composites for applications in the energy, electronic and aeronauticsaerospace industries. The interested reader might find useful complementary information in references [40-43] and a special issue of the Journal of Raman Spectroscopy [44].

- The $1^{\text {st }}$ section outlines the principles of conventional micro-Raman Spectroscopy ( $\mu$ RS).

- The $2^{\text {nd }}$ section introduces the main effects at stake for nanomaterials, with special emphasis on the Phonon Confinement Model (PCM) and the Elastic Sphere Model (ESM) that connect spectral features to "grain size". We made a special effort to select the keystone papers, those which introduced, popularised or significantly improved the different concepts.

- The $3^{\text {rd }}$ section presents the experimental versatility of $\mu \mathrm{RS}$ as applied to nanomaterials (phase identification, phase transition monitoring, grain size determination, defect concentration assessment, etc.).

- The $4^{\text {th }}$ section deals with the mechanical aspects of $\mu$ RS ("Raman extensometry"). Special emphasis is placed on the relationship between the stress-related coefficients $S^{\varepsilon / \sigma}$ and the macroscopic response of materials to the applied stress.

\section{THE FUNDAMENTALS OF RAMAN SPECTROSCOPY}

\section{1- Vibrations in Crystalline Solids}

All collective vibrations that occur in crystals can be viewed as the superposition of plane waves that virtually propagate to infinity [45]. These plane waves, the so-called normal modes of vibration, are commonly modelled by quasi-particles called phonons. A normal coordinate of the form $\mathrm{Q}=\mathrm{Q}_{0} \cos \left(2 \pi \nu_{\mathrm{vib}} \mathrm{t}\right)$, which is actually a linear combination of bond lengths and bond angles, is associated with each normal mode. Depending on the dominant term in the normal coordinate, modes can be classified as either stretching $(v)$, bending $(\delta)$, torsional $(\tau)$ or librational ( $\mathrm{R}^{\prime} / \mathrm{T}^{\prime}$ pseudo-rotations/translations) and lattice modes (the latter include the relative displacement of the unit cells.

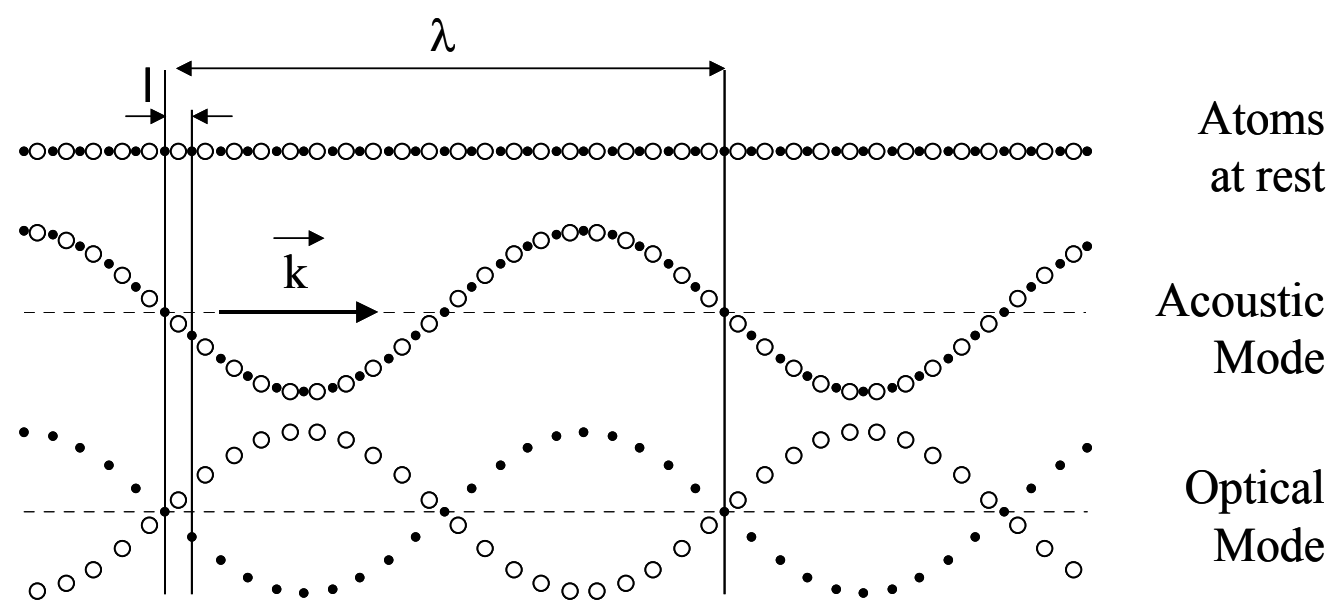

Figure 1 : The transverse phonons $\left(\|\overrightarrow{\mathrm{k}}\|=\frac{2 \pi}{\lambda}\right)$ in a 1D-solid with unit cell parameter 1 . 
For a three-dimensional (3D) solid containing $\mathrm{N}$ unit cells with $\mathrm{p}$ atoms each, $(3 \mathrm{pN}-6)$ different phonons can propagate ${ }^{1}$ and their wavevectors $(\vec{k})$ all point in a volume of the reciprocal space called the Brillouin Zone $(\mathrm{BZ})^{2}$. There are modes with in-phase oscillations of neighbouring atoms and modes with out of phase oscillations. The former are called acoustic vibrations and the latter are called optical vibrations. On the other hand, phonons are referred to as being longitudinal or transversal depending on whether the atoms move parallel or perpendicular to the direction of wave propagation given by $\overrightarrow{\mathrm{k}}$. Phonons with the same two criteria are all gathered in the BZ on $3 p$ (discrete) lines called the dispersion branches (see an example in Figure 17). Figure 1 is an illustration of the concept of phonons in crystals showing the transverse vibrations in a one-dimensional lattice where $\mathrm{p}=2$.

\subsection{The Raman Effect}

The polarization of the dipoles excited in solids when a laser (amplitude $\mathrm{E}_{0}$; frequency $v_{\text {las }}$ ) interacts with phonons (of frequency $v_{\text {vib }}$ ) depends on the polarisability tensor $\overline{\bar{\alpha}}$ :

$$
\overrightarrow{\mathrm{P}}=\overline{\bar{\alpha}} \times \overrightarrow{\mathrm{E}}_{0} \cos \left(2 \pi v_{\text {las }} \mathrm{t}\right)
$$

$\overline{\bar{\alpha}}$ terms can be individually described as functions of the normal vibration coordinates $\mathrm{Q}$ using a Taylor approximation:

$$
\begin{gathered}
\alpha_{\mathrm{ij}}=\alpha_{\mathrm{ij}}^{0}+\left(\frac{\partial \alpha_{\mathrm{ij}}}{\partial \mathrm{Q}}\right)_{\mathrm{Q}=\mathrm{Q}_{0}} \times \mathrm{Q} \quad(\mathrm{i}, \mathrm{j}=\mathrm{x}, \mathrm{y} \text { or } \mathrm{z}) \\
\mathrm{P}_{\mathrm{i}}=\sum_{\mathrm{j}} \alpha_{\mathrm{ij}} \times \mathrm{E}_{\mathrm{j}}=\sum_{\mathrm{j}}\left[\alpha_{\mathrm{ij}}^{0} \mathrm{E}_{0_{\mathrm{j}}} \cos \left(2 \pi v_{\text {las }} \mathrm{t}\right)+\right. \\
\frac{\mathrm{E}_{0_{\mathrm{j}}} \mathrm{Q}_{0}}{2}\left(\frac{\partial \alpha_{\mathrm{ij}}}{\partial \mathrm{Q}}\right)_{\mathrm{Q}=\mathrm{Q}_{0}} \times\left[\cos \left(2 \pi\left(v_{\text {las }}-v_{\text {vib }}\right) \mathrm{t}\right)+\cos \left(2 \pi\left(v_{\text {las }}+v_{\text {vib }}\right) \mathrm{t}\right)\right]+\ldots
\end{gathered}
$$

With the scattered electric field being proportional to $\overrightarrow{\mathrm{P}}$, Eq. (3) predicts both quasi-elastic $\left(\nu \sim v_{\text {las }}\right)$ and inelastic $\left(v=v_{\text {las }} \pm v_{\text {vib }}\right)$ light scattering. The former is called the Rayleigh scattering and the latter, which occurs only if vibrations change polarisability $\left(\partial \alpha_{\mathrm{ij}} / \partial \mathrm{Q} \neq 0\right)$, is the Raman scattering [46,47]. Raman spectroscopists normally refer to vibration modes by their wavenumber $\bar{v}=v_{\text {vib }} / \mathrm{c}$ (c the light speed, $\bar{v}$ in $\mathrm{cm}^{-1}$ unit) and the classical electromagnetic theory of radiations from an oscillating dipole demonstrates that Raman peaks have a Lorentzian shape ${ }^{3}$ :

$$
\mathrm{I}(\overline{\mathrm{v}})=\mathrm{I}_{0} \times \int_{(\mathrm{BZ})} \frac{\mathrm{d}^{3} \overrightarrow{\mathrm{k}}}{[\overline{\mathrm{v}}-\overline{\mathrm{v}}(\overrightarrow{\mathrm{k}})]^{2}+\left(\frac{\Gamma_{0}}{2}\right)^{2}}
$$

\footnotetext{
${ }^{1}$ There are $3 \mathrm{pN}$ degrees of freedom but the 6 rotations and translations of the whole solid are not considered to be proper vibrations.

${ }^{2}$ The BZ describes the geometrical distribution of the wavevectors in the reciprocal space in the same way the unit cell describes the geometry and periodicity of the crystalline arrangement in the direct space.

${ }^{3}$ The experimental bands are a convolution between this natural lineshape, the instrumental transfer function [48,49] and the disorder-induced distribution of vibrators. It is often taken as a Gaussian or a Voigt function (a perfectly symmetric convolution of Lorentzian and Gaussian functions).
} 
In Eq. (4), $\bar{v}(\vec{k})$ represents the dispersion branch to which the mode belongs and $\Gamma_{0}$ is the half-width for the ordered reference structure.

The scattering of one photon $(\overrightarrow{\mathrm{k}} \sim \overrightarrow{0})$ by $\mathrm{n}$ phonons (wavevectors $\overrightarrow{\mathrm{k}}_{\mathrm{i}}$ ) is governed by the momentum conservation rule:

$$
\sum_{\mathrm{i}=1}^{\mathrm{i}=\mathrm{n}} \overrightarrow{\mathrm{k}}_{\mathrm{i}}=\overrightarrow{\mathrm{k}}_{\text {scattered }}-\overrightarrow{\mathrm{k}}_{\text {incident }} \approx \overrightarrow{0}
$$

Therefore, only vibrations from the centre of BZ $(\mathrm{BZc})$, i.e. long wavelength phonons can be active in any one phonon process (first order spectrum) ${ }^{4}$. However, not all BZc phonons are active in RS. According to Eq. (3), $\partial \alpha_{\mathrm{ij}} / \partial \mathrm{Q}$ terms must be different from zero and this condition is governed by the symmetry of the crystals. Raman activity can therefore be predicted through Group Theory [52].

An interesting feature of Eq. (3) is to reveal the dual sensitivity of RS to the electrical $\left(\alpha_{\mathrm{ij}}\right)$ and mechanical $\left(v_{\mathrm{vib}}\right)$ properties of the investigated materials. Two kinds of parameters will therefore influence the spectra:

(i) parameters acting on the "mechanics" like atomic mass, bond strength or the system geometry (interatomic distances, atomic substitutions) will set the peaks positions (the eigenfrequencies of matter vibrations).

(ii) parameters acting on the "charge transfer" (iono-covalency, band structure, electronic insertion) will set intensity, on the basis of the vibration-induced charge variations occurring at the very bond scale ${ }^{5}$.

As polarisability changes for different kinds of bonds, Raman intensity may not be used to quantitatively determine the amounts of different phases. This limitation can sometimes be an advantage since some secondary phase like an enamel pigment [60-62] or carbon in SiC fibers [38] can be detected in a very small quantity (even traces) and its crystalline structure identified [63]. Elements with high atomic numbers and that are situated on the right side of the periodic table (covalent materials in general) are good Raman scatterers whereas ionic structures are difficult to analyse with RS. As for metals, their surface plasmons limit light penetration. Thus, their Raman signal is extremely weak ${ }^{6}$. There are, however, compounds like the superconducting $\mathrm{YBaCuO}$ oxides in which metal atoms produce a strong Raman signal, owing to their covalent bonding in certain directions of the structure $[65,66]$. Some transition metal ions from the $3 \mathrm{~d}$ (chromium) or $4 \mathrm{f}$ (lanthanides) groups produce strong

\footnotetext{
${ }^{4}$ It is actually the case in large and flawless crystals. For such materials, inelastic neutron scattering is the only way to explore the BZ [50,51].

${ }^{5}$ The vibration of charged species is somewhat analogous to a high frequency conductivity. There is for instance a direct link between infrared absorption coefficients $\alpha(\bar{v})$ and the conductivity $\sigma(\bar{v})$ ([53], pp 375 and 391 in Ref. [54]):$$
\alpha(\bar{v})=\frac{4 \pi}{\mathrm{nc}} \sigma(\bar{v})
$$

In Eq. (N1), $\mathrm{n}$ is the refraction index and $\mathrm{c}$ the light speed. There is also a formal equivalence between the Raman Intensity and $\sigma(\bar{v})$ (see page 375 in Ref. [54]) but it has rarely been characterized on experimental Raman spectra [55] :

$$
\mathrm{I}_{\text {Raman }}(\bar{v}) \alpha \frac{\mathrm{n}^{\mathrm{B}}(\bar{v})+1}{\bar{v}} \sigma(\bar{v}) ; \mathrm{n}^{\mathrm{B}}=\text { Bose occupation factor }
$$

The presence of mobile charge careers in ionic conductors can be identified by Raman bands with temperature dependent intensity [56,57]. More discussion on the ionic motions can be found in papers by Funke et al. $[58,59]$.

${ }^{6}$ Hexagonal close-packed metals like Be are an exception [64].
} 
fluorescence signals which often mask the Raman spectra but can be used for short range structure [67] and/or residual stress [68] assessment.

\subsection{Conventional Raman Spectrometers}

Figure 2-a shows the principle of a Raman spectrometer. Up-to-date equipment would include holographic gratings, for improved excitation light rejection, a set of monochromators and a liquid nitrogen- or Peltier effect-cooled CCD mosaic for detection [46,69]. The laser source is often built-in but light coming from an external excitation source can also be used. In the "macro"-configuration, the beam section is $\sim 1 \mathrm{~mm}^{2}$ but the laser spot can be reduced to $\sim 1$ $\mu \mathrm{m}$ diameter by using the high-magnification microscope objectives with which most commercial Raman spectrometers are equipped. The technique involved is known as microRaman Spectroscopy $(\mu R S)$. The main additional options are motorized stages for $\mathrm{XY}(\mathrm{Z})$ mappings and optical fibre plugs for connection to remote optical heads equipped with microscope objectives [70].

a)

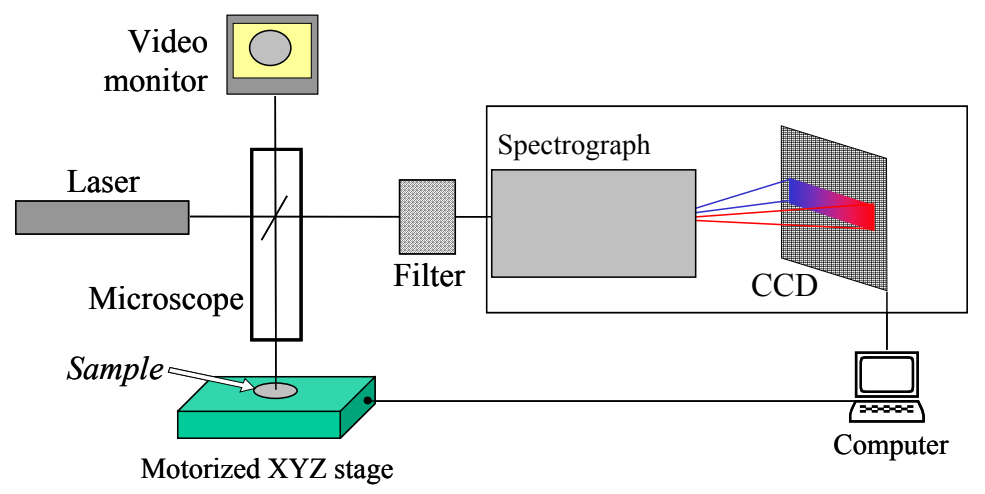

b)

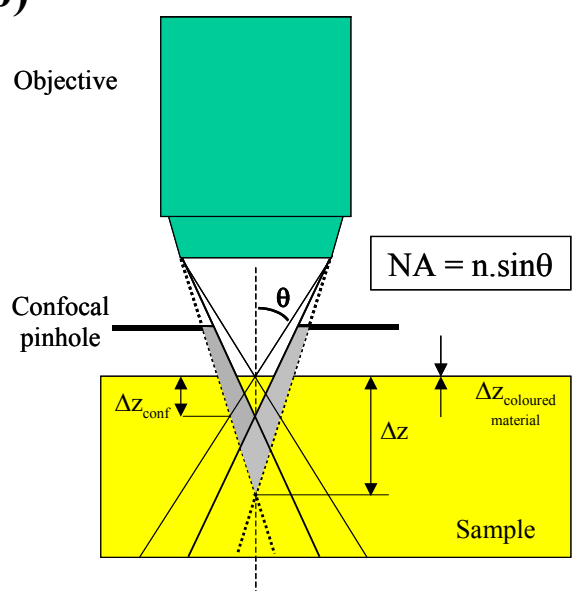

Figure 2 : a) Principle of a conventional micro-Raman spectrometer - b) Observation of a sample through a microscope (NA: Numerical Aperture; $n$ is the refractive index of the medium separating the objective from the sample). A confocal hole rejects the shadowed light and facilitates a more accurate in-depth analysis $\left(\Delta \mathrm{z}_{\text {conf }}<\Delta \mathrm{z}\right)$.

Raman maps are images generated from spectra recorded at discrete points of the sample (the recording is automated). They show the variation of any fitted parameter (i.e. intensity, width or position of one band) as a function of the point of analysis. If the mapping is regular and sufficiently tight, one gets a "smart map" of the parameter (colour or contrast scaling) superimposed with the optical image of the probed area (see Figure 14-b) [39]. Raman parameters can thus be correlated with the crossover from one specific region (phase) to another.

Raman mapping is not to be mistaken for direct Raman imaging where a large area of the sample is probed all at once and no fitting is required. More precisely, only photons from a narrow spectral domain are sent to the CCD mosaic and each pixel receives those coming from a given area of the sample. The intensity of the signal thus reveals the presence and location of any substance with a strong Raman signal in the selected spectral window. This is used by customs services to search for drugs hidden in permissible powders like sugar [71]. 


\subsection{Lateral and "in-depth" Resolution of Conventional $\mu R S$}

Owing to the diffraction of light, the intensity coming from a point observed through a microscope is distributed over an "Airy disk". The lateral resolution R, which is the smallest distance between two points to still appear distinctively on the microscope image, is half the width of the Airy disks. According to the Rayleigh criterion [72]:

$$
\mathrm{R}=\frac{0.61 \times \lambda}{\mathrm{NA}}
$$

In Eq. (6), $\lambda$ is the light wavelength and NA represents the numerical aperture (see Figure 2b). As for the axial resolution $(\Delta z)$ of $\mu \mathrm{RS}$, an estimate is given by the depth of field, which is defined as half the width of the axial intensity profile. Born and Wolf came up with a famous analytical expression for $\Delta z$ [73], which is well approximated by a simpler expression by Conrady [74]:

$$
\Delta \mathrm{z}=\frac{\lambda}{\mathrm{n} \sin ^{2} \theta} \Rightarrow \Delta \mathrm{z}_{\substack{\text { through air } \\(\mathrm{n}=1)}}=\frac{\lambda}{\mathrm{NA}^{2}}
$$

Under "standard" conditions $(\mathrm{n}=1, \lambda=500 \mathrm{~nm}, \mathrm{NA}=0.5)$, the typical lateral and in-depth resolutions of $\mu \mathrm{RS}$ are 1 and $2 \mu \mathrm{m}$, respectively. Even with the smallest visible wavelength ( $400 \mathrm{~nm}$ ) and the highest numerical apertures (oil immersion objectives with $\mathrm{n}=1.515$; NA 1.4), one should not expect a lateral resolution better than $\mathrm{R}=0.2 \mu \mathrm{m}$ (the Abbé criterion states that the wavy nature of light forbids to distinguish points closer than $\lambda / 2$ ) and a field depth below $\Delta \mathrm{z}=0.4 \mu \mathrm{m}[75]$.

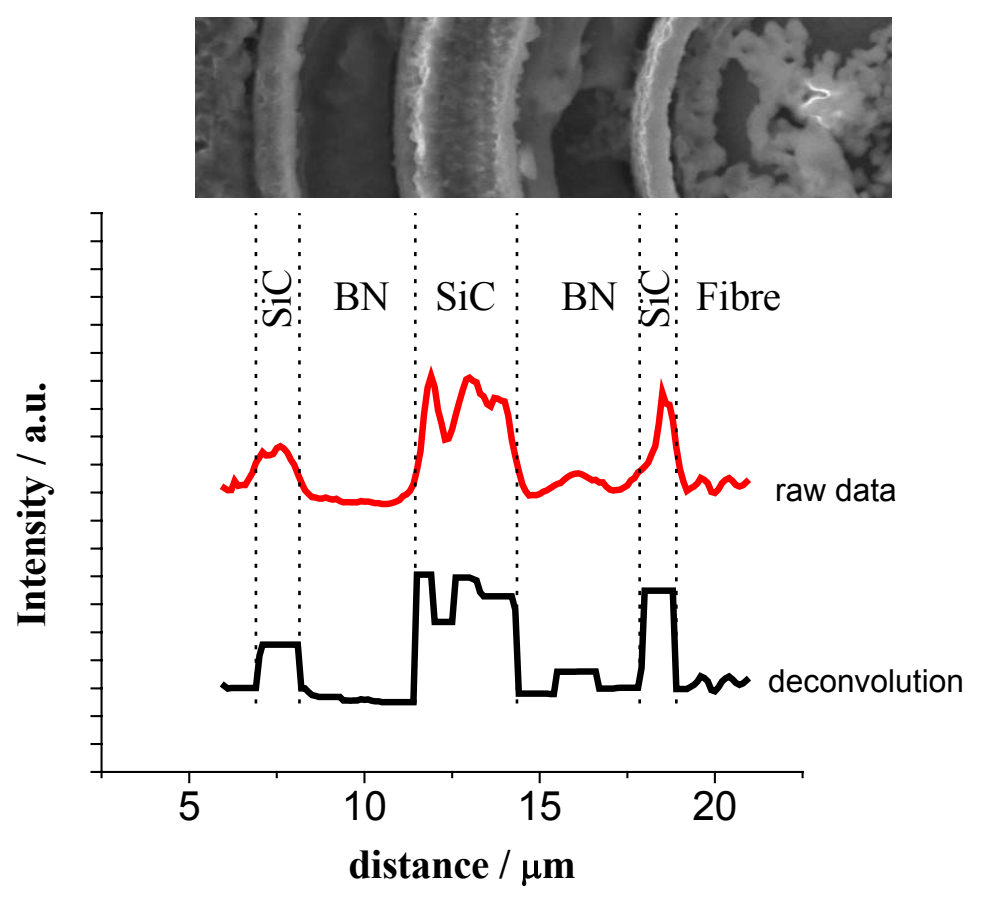

Figure 3 : The intensity profile obtained from a step by step Rayleigh mapping of a multilayered $\mathrm{SiC} / \mathrm{BN}$ coating deposited on a Hi-Nicalon fibre $(\lambda=632.8 \mathrm{~nm}, \mathrm{Obj} . \times 100$ Olympus MSPlan ULND, NA=0.80) can be deconvoluted by a function (shown in Ref. [76]) characterizing the distribution of energy in the spot (Adapted from $\mathrm{Ph}$. Colomban,

Spectroscopy Europe, 15[6], pp. 8-16, 2003). 
However, if a series of spectra are recorded at very close equidistant locations (X-Y stages commonly have a displacement resolution of one tenth of a micron), a reduced "effective" spot size is obtained through a convolution of the spot profile with the displacement step (see Figure 3 generated from a Rayleigh scattering mapping procedure; similar conclusions would apply to Raman images). Even then, there is still a high number of "nano-sources" contributing to the Raman signal.

\subsection{Resonant Raman Spectroscopy (RRS)}

If the energy of the laser excitation (usually in the UV-nIR range) is high enough to approach those of the various electronic states of the material (in other words if the material is coloured), Raman spectra may be mingled with photoluminescence spectra arising from excited electronic levels. The consequence is the strong enhancement of some vibrational modes (near-resonance/resonance Raman scattering) but one should notice that the probed chemical bonds are in a markedly disturbed state [22,26,27,77-79]. The light penetration is reduced to a few tens of nanometers only $\left(\Delta z_{\text {coloured }}\right.$ in Figure $\left.2-b\right)$, which makes $\mu$ RS a good method for surface analysis [23,80] $]^{7}$. If films or fibre-reinforced composites made of absorbent materials are polished with surface to growth direction and surface to fibre axis angles slightly different from, respectively, 90 and $0^{\circ}$, then in-line scans of the samples provide high spatial resolution across the film thickness [84-86] or the fibre-matrix contact region (interphase) [22].

The penetration depth $\delta$ of the light is directly related to the wavelength-dependent coefficient of linear absorption $\mathrm{a}_{1}\left(\mathrm{~cm}^{-1}\right)$ :

$$
\delta=\frac{\lambda_{\text {excitation }}}{4 \pi \mathrm{nk}}=\frac{1}{\mathrm{a}_{1}(\lambda)}
$$

In Eq. (8), $\mathrm{n}$ and $\mathrm{k}$ are respectively the refraction and extinction indexes. $\delta$ is indeterminate in the (frequent) lack of absorption coefficients but switching excitation wavelengths close to the electronic absorption threshold can help separate surface from bulk Raman contributions [37]. This is illustrated in Figure 4, where polyaniline fibres spectra recorded with red, green and blue laser lines, are compared [87]. The bottom spectrum corresponds to the surface of these fibres (maximum electronic absorption) where a "type I" monoclinic form of polyaniline is dominant [88]. By contrast, the bulk of the fibre (as seen with blue excitation on the top spectrum) contains more of the type II orthorhombic form. Following the same principle, Shen and Pollak [49] or Yakimova et al. [48] used multiple laser lines to measure spectra at different depths below the surface of semiconductor films.

Note that when the absorption is very high, temperature may rise at the point of laser impact, even for a few $\mu \mathrm{W} / \mu \mathrm{m}^{2}$ irradiation. This effect is reduced if the sample is either dispersed in a non-absorbing matrix or put in a rotating cell or a low temperature cryostat but often results in a wavenumber shift or even a chemical degradation of the sample (oxidation, decomposition, etc.). It is then mandatory to calibrate the thermal effects [89-91]. Besides, whenever the absorbing phase is not dispersed in a transparent matrix, a significant part of the scattered light intensity may be reabsorbed.

\footnotetext{
${ }^{7}$ Resonance Raman spectra recorded with different wavelengths of excitation are also a way of characterizing excitons and polarons in semiconductors $[81,82]$ and conducting polymers $[83]$.
} 


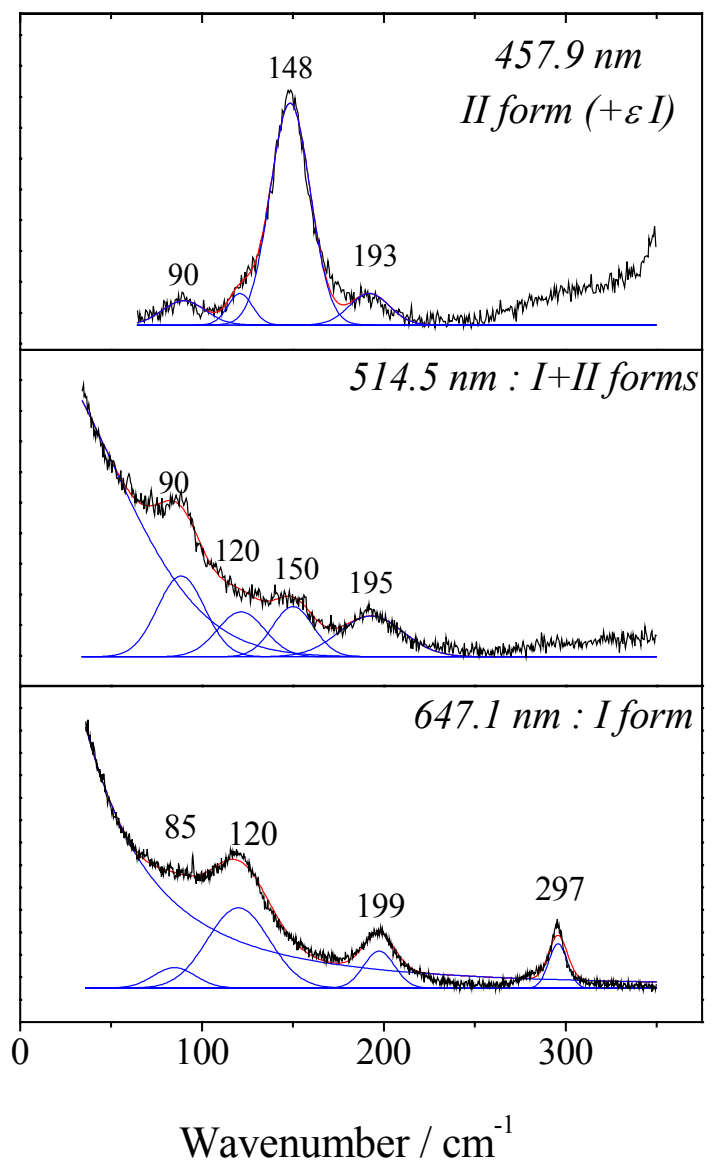

Figure 4 : Raman spectra recorded on polyaniline/Camphor Sulfonic Acid fibres. The use of three different excitation wavelengths modifies the probing depth (Adapted from A. El-Khalki et al., Synthetic Metals, 139[2], pp 215-220, 2003).

\subsection{Analysis of "Isolated" Units: the Molecular Scheme}

Eq. (4) corresponds to a description where vibrations in solids are pictured as collective waves but another description applies to solids with bonds of different strengths. In this "molecular" description, clusters of strong covalent bonds are isolated from one another by weaker ionic bonds and, thus, become the relevant vibrational unit (localized vibrations). All atoms from this unit must exclusively belong to it (including, for example, oxygen atoms from polymerised oxides) for the normal coordinates of Eq. (2) \& (3) to coincide with given bond lengths and bond angles. The spectra then reveal stretching and bending modes equivalent to those of polyhedral (mostly tetrahedral or octahedral) isolated molecules [9295]. The difference arises from the cations generating $\mathrm{T}^{\prime}$ (translational) and $\mathrm{R}^{\prime}$ (rotational) libration modes. The molecular scheme describes most organic polymers but also crystalline/amorphous inorganic polymers such as silicates, phosphates, niobiates, titanates [96-98] and all compounds with polyatomic cations such as $\mathrm{NH}_{4}{ }^{+}, \mathrm{H}_{3} \mathrm{O}^{+}$or $\mathrm{N}_{2} \mathrm{H}_{5}{ }^{2+}$ (page 379 in Ref. [54]). A given vibration then always appears in the same region, its exact position giving information about the local environment of the corresponding bonds, both in the crystalline and amorphous states [94,99-104]. Yet, these bonds are probed with a one square micrometer section beam, which means one gets only an average view over their distribution. 


\section{7 "Nano-specific" Modes}

There are two ways of truly isolating the Raman signal coming from nano-particles. One is by having a nanoparticle to be the only one of its kind in the laser's path (SERS) while the other involves a breaking of the $\lambda / 2$ diffraction limit of optical microscopes (nano-Raman).

\subsection{1 $\rightarrow$ Surface-Enhanced Raman Spectroscopy (SERS)}

The Raman signal may be amplified by several orders of magnitude for molecules adsorbed on roughened surfaces [105,106], colloid particles [107] and nanowires [108] of transition metals (mostly silver). This technique is called Surface-Enhanced Raman Spectroscopy (SERS) and the amplification results from the interaction between the electromagnetic field of the laser excitation and the surface plasmon of the metal. This enhancement may be so high that a signal can be recorded with just one or a few particles being probed simultaneously. Unfortunately, getting the right conditions for SERS requires much sample preparation and additional measurements are often necessary to interpret the SERS data collected $[107,109]$. SERS is seldom applied to solid films [110] and is mainly used in Biology, where "molecules" must be sufficiently diluted to follow their interactions (e.g. a protein with its "Redox" partner [111]). Roy et al. used SERS for a specific study of the surface carbon in Hot Filament CVD carbon $[112,113]$. Azoulay et al. also demonstrated the possibility of selecting single wall carbon nanotubes (SWCNTs) by SERS [114].

\section{$\underline{2.7 .2 \rightarrow \text { Nano-Raman }}$}

In Near-Field Scanning Optical Microscopy (NSOM in the US; SNOM in Europe), the $\lambda / 2$ diffraction limit of optical microscopes (Abbé criterion) is surpassed thanks to the addition of a small aperture made at the end of a tapered probe, frequently a metal-coated optical fibre tip. This confines the optical field and thus imposes the lateral resolution [115]. The probe must be kept extremely close to the sample using micro-manipulation tools borrowed from Atomic Force and Scanning Tunneling Microscopes (AFM/STM) and the technique becomes NSOM-Raman or, simply, "nano-Raman", when a NSOM equipment is coupled with a Raman spectrometer [116-124]. Even under the most favourable operating conditions, the excitation is reduced by the optical fibre cut-off and only a faint signal is collected from the small volume that is excited. This is why nano-Raman proved to be efficient only with very good Raman scatterers [120,121,123]. More recently, SERS capability was implemented on nano-Raman equipments by the addition of a vibrating apertureless metallic tip brought close to the surface of the sample [125-128]. This is called Tip Enhanced Raman Spectroscopy (TERS). The intensity enhancement varies in $\mathrm{d}^{-12}$ ( $\mathrm{d}$ being the probe-sample spacing) [129] and getting a good TERS signal is thus far from trivial.

SERS and nano-Raman will not be further discussed in this review. The focus shall instead be placed on the nano-related information that can be retrieved using conventional $\mu$ Raman spectrometers that have nowadays become standard in a number of research and industrial laboratories, owing to the availability of convenient commercial instruments. 


\section{THE VIBRATIONAL SPECTRA OF NANOMATERIALS}

The translational symmetry of crystalline materials is broken at grain boundaries, which results in the appearance of specific surface and interface vibrational contributions [130]. Besides, the grains outer atomic layers often react with neighbouring species (lattice reconstruction, passivation/corrosion layers, contamination) and experience steep thermochemical gradients during processing, which generates new phases, with their own spectral contributions. These two elements are often neglected in RS but we can expect them to become very significant in nano-crystals, where the concentration of grain boundaries is very high.

\subsection{Phase Identification and Phase Transitions in Nanoparticles}

In many nanomaterials, the Raman spectrum remains sufficiently similar to that of the corresponding single crystal to facilitate direct identification of the phases [28,32,131-146]. Once the Raman spectra are known, phase transitions can be characterized (transition temperature, transition pressure, transition order) through mode variation, much the same way as in bulk materials [93,138,147-159]. Besides, the observation of any theoretically forbidden mode is a very sensitive probe of lattice distortions $[132,160]$.

Barborini and co-workers showed with RS that the structure of gas phase-deposited $\mathrm{TiO}_{2}$ clusters turned from rutile to anatase whenever they reached $5 \mathrm{~nm}$ in diameter [158]. A difference in surface energy usually plays a determinant role in such phenomenon, as proposed a long time ago by R.C. Garvie for zirconia [161]. Similarly, Fray and Payne showed how the temperature of the orthorhombic-tetragonal phase transition of $\mathrm{BaTiO}_{3}$ ceramics depended on the grain size [159].

\section{2- Analysis of Amorphous Domains}

Micro Raman spectroscopy is sometimes more powerful than X-ray analysis for detecting and monitoring crystallisation/amorphisation [93,101,147,162-165] in covalent materials. Of course, both crystallographers and Raman spectroscopists characterize disorder through peak broadening. Yet, while a loss of long distance translational periodicity (of high atomic number atoms) is always associated with broadening in the case of diffraction patterns, only lattice and librational (R', T') modes are sensitive to the same "long distance" disorder in RS [166]. The width of the other Raman modes is mainly sensitive to the "local" crystal field, more specifically the short range order in the first (0.1-0.5 nm) and second (0.5-5 nm) atomic shells. If the "molecular" description of vibrations applies (see §2.6), then Raman bending modes are even specifically sensitive to local geometric disorientation and Raman stretching modes to the neighbouring disorder (particularly atoms from other sublattices or electric defects resulting from substitutions/vacancies).

In fact, diffraction discriminates "periodical" domains from "disordered" ones but does not easily differentiate clear-cut separations in the real space and progressive orientational disorders (para-crystal), especially in strongly covalent structures such as organic and inorganic polymers (pp. 615-620 in Ref. [167]). In materials, mainly polymers, which present 
interlocking submicronic "crystalline" and "amorphous" conformational domains [168-175], the distinction is sometimes possible using $\mu \mathrm{RS}$. The simplest way to picture the problem is to fit lattice modes with two components, one representing the amorphous state and the other the crystalline state. The area ratio of the two underlying areas yields a good estimate of crystallinity [176,177]. Figure 5 illustrates this point with a spectrum of the polyamid 6.6 fibre. Polarized analysis clearly shows the orientational effect of straining (fibre extrusion) on the nanocrystals whereas the specific analysis of the low frequency components, which show the collective chain movements, facilitates the separate analysis of the amorphous (wide Gaussian band) and crystalline (narrow Lorentzian) phases. These results were used to demonstrate that the mechanical fatigue of the fibres results from the progressive transformation of the amorphous phase [170,171,173]. Nanophase separation was also investigated in glasses where the connectivity of constitutive polyhedra sets the wavenumbers [178]. Some bands could be attributed to definite clusters on the basis of comparison with experimental spectra of reference crystalline phases [178] or first principle Density Functional Theory (DFT) calculations [95,179].

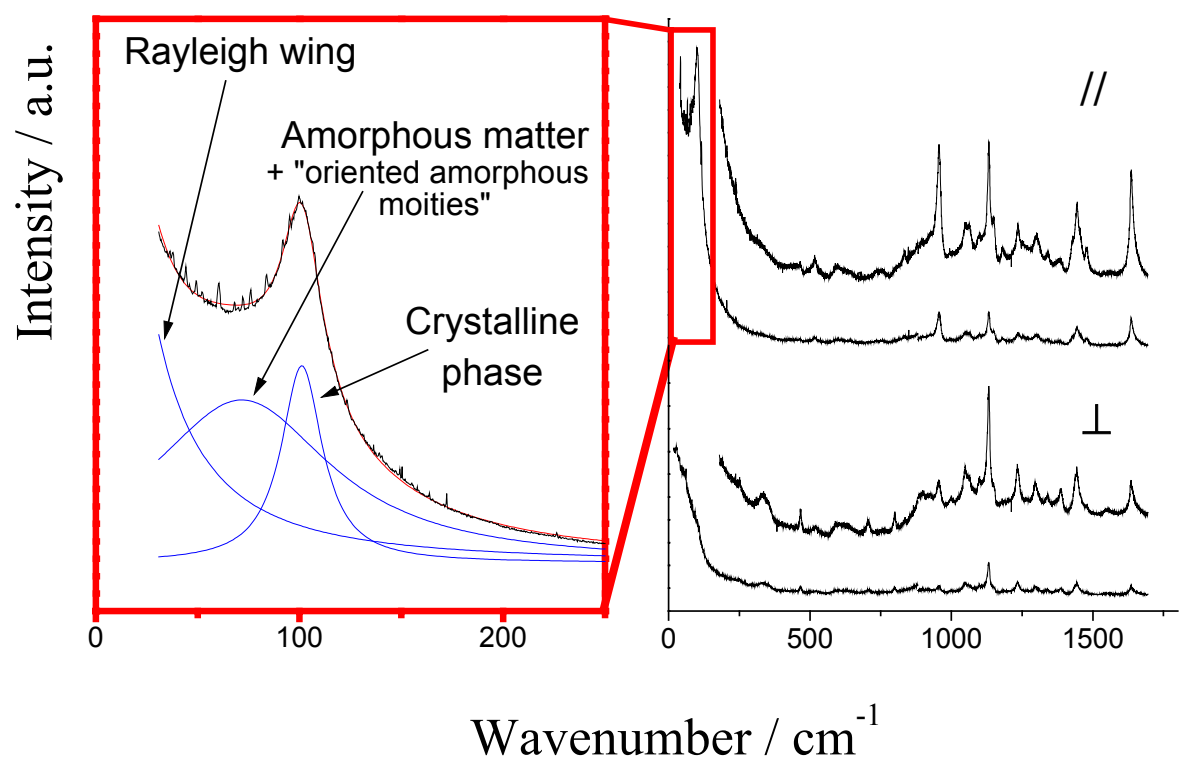

Figure 5 : Low wavenumber Raman spectra of the PA 66 polyamide fibre (FUHP grade, Rhodia) recorded with the exciting electric field polarized either parallel $(/ /)$ or perpendicular $(\perp)$ to the fibre axis $\left(\lambda_{\text {exc }}=514.5 \mathrm{~nm}\right)[172]$. The collective mode at $\sim 100 \mathrm{~cm}^{-1}$ is highly polarized. The zoom shows how the crystalline and amorphous (much wider) contributions can be analysed separately [170].

\subsection{Size Determination in Nanomaterials}

Two models are widely used to derive particle size from Raman spectra: the Phonon Confinement Model (PCM) projects Raman "inactive" bulk modes onto the BZc whereas the Elastic Sphere Model (ESM) describes the free oscillations of homogeneous spheres.

\section{$\underline{3.3 .1 \rightarrow}$ The Phonon Confinement Model (PCM)}

Richter et. al. proposed a very intuitive Phonon Confinement Model (PCM) for the phonons in nanospheres of diameter L [180]. They simply multiplied the plane wave describing a 
phonon, with wavevector $\overrightarrow{\mathrm{k}}_{0}$, in a perfect crystal $\left(\Phi\left(\overrightarrow{\mathrm{k}}_{0}, \overrightarrow{\mathrm{r}}\right)=\mathrm{u}\left(\overrightarrow{\mathrm{k}}_{0}, \overrightarrow{\mathrm{r}}\right) \mathrm{e}^{i \overrightarrow{\mathrm{k}}_{0} \cdot \overrightarrow{\mathrm{r}}}\right.$, u having the same spatial periodicity as the lattice) by a Gaussian function:

$$
\Phi\left(\overrightarrow{\mathrm{k}}_{0}, \overrightarrow{\mathrm{r}}\right)=\mathrm{e}^{-\alpha\left(\frac{\mathrm{r}}{\mathrm{L}}\right)^{2}} \times \mathrm{u}\left(\overrightarrow{\mathrm{k}}_{0}, \overrightarrow{\mathrm{r}}\right) \mathrm{e}^{\mathrm{i} \overrightarrow{\mathrm{k}}_{0} \cdot \overrightarrow{\mathrm{r}}}
$$

Assuming $\mathrm{u}\left(\overrightarrow{\mathrm{k}}_{0}, \overrightarrow{\mathrm{r}}\right) \sim \mathrm{u}(\overrightarrow{\mathrm{r}})$ and using FT to refer to Fourier Transforms, Eq. (9) is equivalent to:

$$
\begin{gathered}
\Phi\left(\overrightarrow{\mathrm{k}}_{0}, \overrightarrow{\mathrm{r}}\right) \propto \mathrm{FT}^{-1}\left[\mathrm{FT}\left(\mathrm{e}^{-\alpha\left(\frac{\mathrm{r}}{\mathrm{L}}\right)^{2}} \times \mathrm{e}^{\mathrm{i} \overrightarrow{\mathrm{k}}_{0} \cdot \overrightarrow{\mathrm{r}}}\right)\right]=\int \mathrm{d}^{3} \overrightarrow{\mathrm{k}} \mathrm{C}\left(\overrightarrow{\mathrm{k}}_{0}, \overrightarrow{\mathrm{k}}\right) \cdot \mathrm{e}^{\mathrm{i} \overrightarrow{\mathrm{k}} \cdot \overrightarrow{\mathrm{r}}} \\
\text { with } \mathrm{C}\left(\overrightarrow{\mathrm{k}}_{0}, \overrightarrow{\mathrm{k}}\right)=\frac{1}{(2 \pi)^{3}} \int \mathrm{d}^{3} \overrightarrow{\mathrm{r}}\left(\mathrm{e}^{-\alpha\left(\frac{\mathrm{r}}{\mathrm{L}}\right)^{2}} \mathrm{e}^{\mathrm{i}\left(\overrightarrow{\mathrm{k}}_{0}-\overrightarrow{\mathrm{k}}\right) \cdot \overrightarrow{\mathrm{r}}}\right)
\end{gathered}
$$

Thus, the wave associated with a phonon confined in an imperfect crystal simply is a superposition of plane waves with $\left|\mathrm{C}\left(\overrightarrow{\mathrm{k}}_{0}, \overrightarrow{\mathrm{k}}\right)\right|^{2}$ weight. Recalling that each wave gives rise to a Lorentzian (Eq. (4)), Raman intensity is eventually given by:

$$
\mathrm{I}(\overline{\mathrm{v}}) \alpha \int_{\mathrm{BZ}} \mathrm{d}^{3} \overrightarrow{\mathrm{k}} \frac{\left|C\left(\overrightarrow{\mathrm{k}}_{0}, \overrightarrow{\mathrm{k}}\right)\right|^{2}}{[\overline{\mathrm{v}}-\overline{\mathrm{v}}(\overrightarrow{\mathrm{k}})]^{2}+\left(\frac{\Gamma_{0}}{2}\right)^{2}}
$$

Eq. (12) mathematically expresses the Raman selection rule breaking induced by phonon confinement $^{8}$ with a weighed exploration of the dispersion curve. Taking $\mathrm{k}_{\mathrm{BZe}}$ as the edge of $\mathrm{BZ}$, $\mathrm{q}$ as the reduced wavevector $\left(\mathrm{q}=\mathrm{k} / \mathrm{k}_{\mathrm{BZe}}\right)$ and assuming isotropic mode dispersion [181], Eq. (12) yields:

$$
\mathrm{I}(\overline{\mathrm{v}}) \alpha \int_{\mathrm{q}=0}^{\mathrm{q}=1} \mathrm{dq} \mathrm{e}^{-\frac{\mathrm{k}_{\mathrm{Bze}}^{2}\left(\mathrm{q}-\mathrm{q}_{0}\right)^{2} \mathrm{~L}^{2}}{2 \alpha}} \times \frac{1}{[\overline{\mathrm{v}}-\overline{\mathrm{v}}(\mathrm{q})]^{2}+\left(\frac{\Gamma_{0}}{2}\right)^{2}}
$$

This is the equation to which most authors refer when using the PCM [180-187] ${ }^{9}$. For Quantum Dots (QDs), NanoWires (NWs) or slabs, the PCM is easily adapted using the appropriate expressions for the $d^{3} \vec{k}$ integration volume in Eq. (12) [190-194] $]^{10}$. Knowledge of

\footnotetext{
${ }^{8}$ As a consequence of Heisenberg's uncertainty principle, the uncertainty $\Delta \mathrm{k}=\Delta \mathrm{p} / \mathrm{h}$ on the wavevector must remain above or equal to $(2 \mathrm{~L})^{-1}$.

${ }^{9}$ Based on the triple hypothesis that modes from different crystallites are uncorrelated, that lifetimes can be simulated with Lorentzian broadening $\left(\Gamma_{0}\right)$ and that susceptibility variations $(\Delta \chi)$ are proportional to the normal vibrations coordinates, Nemanich, Solin and Martin [188] predicted the Raman intensity on probing of $N$ orthorhombic crystallites to be:

$\mathrm{I}(\overline{\mathrm{v}}) \alpha \mathrm{S}\left(\overrightarrow{\mathrm{k}}_{0}, \overline{\mathrm{v}}\right)=\frac{\mathrm{N}}{\mathrm{c}^{2} \mathrm{~V}^{2}}\left(\frac{\mathrm{n}^{\mathrm{B}}(\overline{\mathrm{v}})+1}{\overline{\mathrm{v}}}\right) \mathrm{V}^{-1} \sum_{\overrightarrow{\mathrm{k}}, \mathrm{j}} \mathrm{C}\left(\overrightarrow{\mathrm{k}}, \overline{\mathrm{v}}_{\mathrm{j}}(\overrightarrow{\mathrm{k}})\right)\left|\mathrm{F}\left(\overrightarrow{\mathrm{k}}-\overrightarrow{\mathrm{k}}_{0}\right)\right|^{2} \times \frac{\Gamma_{0} / 4 \pi}{\left[\bar{v}-\bar{v}_{j}(\overrightarrow{\mathrm{k}})\right]^{2}+\Gamma_{0}^{2} / 4}$

In this Equation, $\mathrm{S}$ is the Fourier transform of polarisability variations associated with Raman scattering, $\left(\mathrm{n}^{\mathrm{B}}(\bar{v})+1\right)$ is the Bose population factor $\left(n^{B}(\bar{V})=1 /\left(e^{h v / k T}-1\right)\right), C$ is the Raman coupling coefficient for branch $\mathrm{j}$ and $\left|\mathrm{F}\left(\mathrm{k}-\mathrm{k}_{0}\right)\right|^{2}$ is the uncertainty on the wavevector induced by phonon confinement $[188,189]$. This approach is entirely equivalent to the PCM if F function is assumed to be Gaussian and the occupation term is neglected (this simplification is justified when only one band is modeled).

${ }^{10}$ Note that the PCM failed to predict the Raman shift in silicon spherical or columnar nanocrystals. Zi et al. showed with a Bond Polarisability Modelling that it could rather be predicted using the following expression [195]:
} 
the Vibrational Density of States (VDOS) is required for computing Eq. (13) and is obtained either from neutron scattering measurements, from data on structurally parent structures or from $a b$ initio calculations based on a rigid-model structure [183,184,196-198]. The PCM, which does not apply to acoustic modes (their energy is nil at BZc) and is very seldom used with TO modes (on account of their low dispersion [193,199]), is almost exclusively applied to LO modes. With LO dispersion usually being maximum at BZc, the integration in Eq. (13) introduces additional contributions on the low frequency side of the single crystal mode and the resulting peaks are asymmetric. Peak adjustment is not always mandatory once phonon confinement has been invoked. In first approximation, the peak shift is indeed proportional to the inverse of the grain size [188,200]. The overall half-width at half-height also is, as was reported for nanocrystalline $\mathrm{CeO}_{2}[197,198]$ (see Figure 6) or boron nitride [188] and can be verified with data on $\mathrm{Ge}$ [185].

In the paper introducing the PCM [180], Richter et al. took $\alpha=2$. Fauchet and Campbell later tested several forms for the weighing function introduced in Eq. (9) [194]. They came to acknowledge the choice of a Gaussian form but chose the more confining parameter $\alpha=8 \pi^{2}$ first proposed by Tiong et al. [199], which corresponds to a rigid confinement hypothesis $\left(\left|\mathrm{C}\left(\overrightarrow{\mathrm{k}}_{0}, \overrightarrow{\mathrm{k}}\right)\right|^{2}=0\right.$ for $\left.\mathrm{r}=\mathrm{L} / 2\right)[181,184,186,198,201]$. A bond polarisability modelling of vibrations in silicon nanocrystals also returned a value of 9.67 for $\alpha$ [202]. As a matter of fact, $\alpha$ may be considered, with L, like an adjustment variable of the model [185]. Besides, L should not systematically identify with the grain size. Phonons can indeed be confined by any "spatially limiting" feature in the grain (twins, stacking faults, inclusions, vacancies, boundaries, pores, etc.). $\mathrm{L}$ is therefore equal to $2 \pi / \Delta \mathrm{k}$, where $\Delta \mathrm{k}$ is the extension of $\mathrm{BZ}$ domain of which the modes are activated. It is nothing but a coherence length which can correspond to a grain size [180,192,203-206], defects/impurities interspacing [186,197,201,207,208], the extension of cation-ordered domains in incommensurate phases [92], that of undamaged crystalline domains in ion-implanted GaAs [199], the size of polytypic domains [182,209,210] or clusters in semiconducting alloys [183,184,211] and ferroelectrics [12]. The physical interpretation of $\mathrm{L}$ therefore is a key element. For instance, Weber thought it was the grain size in $\mathrm{CeO}_{2}$ but found an order of magnitude discrepancy with results from electronic microscopy [198]. In fact, Kosacki et al. showed L was the distance between defects in the oxygen lattice of ceria (see Figure 18-b and attached comments) [197]. Carles et al. showed that the meaning of $\mathrm{L}$ even changes in germanium particles depending on their size. $\mathrm{L}$ corresponds to the actual size for the bigger grains, but represents the domain size in smaller grains where alloying has taken place [181]. See also Fig 16 and the discussion for SiC "grain size".

Although Richter et al. took $\overrightarrow{\mathrm{k}}_{0}$ at the BZc, their model did not preclude it from standing somewhere else (which many authors have forgotten). Adding this degree of freedom to the model finds a physical justification in at least two situations. First, when nanoparticles are surrounded by a parent material, the phonons can be reflected by grain boundaries and their interferences can "activate" modes away from BZc. This effect was mentioned by Arora and co-workers for CVD-diamond particles embedded in amorphous carbon. They fitted the spectra with as many as 20 contributions calculated with Eq.(13) at specific values of $\overrightarrow{\mathrm{k}}_{0} \neq \overrightarrow{0}$

$\bar{v}_{\text {vib }}=\bar{v}_{\text {bulk }}-\mathrm{A}\left(\frac{\mathrm{a}}{L}\right)^{\gamma}$

In equation (N4), a is the lattice constant whereas A and $\gamma$ fully characterize the nanocrystal geometry. 
[212]. Second, in their "Random Stacking Fault" model of disorder in silicon carbide, Rohmfeld et al. used a Bond Polarisability Model (BPM) to calculate the influence of stacking defects occurring with $\Delta \mathrm{L}$ interspacing $[182,209]$. They concluded to the extinction of BZc modes to the benefit of modes with wavevectors equal to $\mathrm{k}_{\mathrm{m}}=(2 \mathrm{~m}+1) \pi / \Delta \mathrm{L}$; $\mathrm{m}=0,1,2 \ldots$, which is analogous to the application of Eq. (13) at $\mathrm{k}_{\mathrm{m}}$ points. Even though disorder and nanosize effects often participate together to phonon confinement, their relative contributions can theoretically be isolated whenever $\overrightarrow{\mathrm{k}}_{0}$ is allowed to vary:

- disorder will disturb short range order and fold the BZ. $\overrightarrow{\mathrm{k}}_{0}$ will be away from BZc and L should then be inversely proportional to the density of defects.

- if the confinement results from the existence of domains in which order is perfect, typically nanocrystals, then the activated modes should be centred in $\Gamma\left(\overrightarrow{\mathrm{k}}_{0}=\overrightarrow{0}\right)$ and L will represent the grain size.

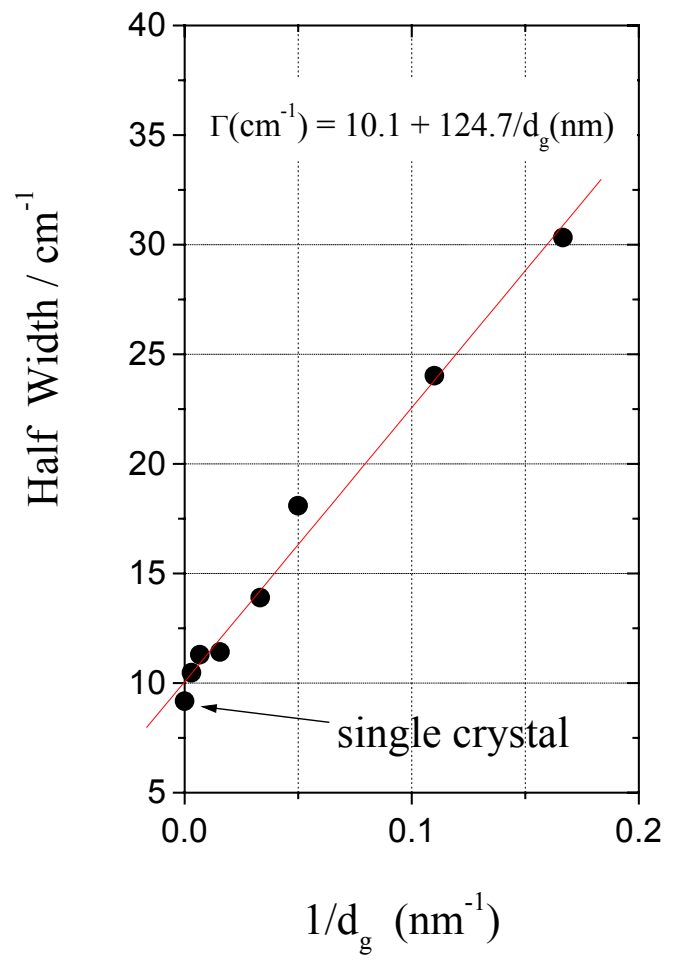

Figure 6 : The half-width at half height of $\mathrm{CeO}_{2}$ Raman band $\left(466 \mathrm{~cm}^{-1}\right)$ plotted as the inverse of the grain size obtained by X-ray diffraction. Reprinted from Solid State Ionics, 149[1-2], I. Kosacki et al., Raman scattering and lattice defects in nanocrystalline $\mathrm{CeO}_{2}$ thin films, pp 99-105, 2002, with permission from Elsevier.

In Reference [180], Richter et al. attributed the Raman signal not accounted for by the PCM to fully amorphous matter (they actually worked on microcrystalline silicon). This explanation was later used by several authors [192,201,213-215] but should not hide the fact that the PCM is based on approximations and neglects some peculiarities of vibrations in nanomaterials :

i) the PCM supposes isotropy: As is, the PCM only applies to materials with homogeneous structure in all directions of space (spherical model). An "isotropic dispersion hypothesis" is 
probably acceptable for almost any material in the limit of $\overrightarrow{\mathrm{k}}_{0}$ close to BZc [185] but integrating Eq. (13) along the main directions of the BZ returns more satisfactory results [216]. Carles et al. proposed a way of simply taking anisotropy into account [181]: all directions are plotted on the same reduced wavevector axis and wavenumbers are simply averaged. As for $\Gamma_{0}$, it is adjusted with an anisotropy parameter based on the maximum branch "splitting" observed between different directions.

ii) the PCM supposes uniform size and shape: Another approximation is made with the PCM when a Gaussian weighing function is introduced in Eq. (9). This assumes all crystallites have the same shape -either spherical [180] or orthorhombic [188]- and size, whereas the actual distributions must be taken into account in a model describing reality $[185,193,203,217,218]$.

iii) the PCM neglects surface/interface phonons: The PCM progressively loses its relevance when the particle size decreases and the surface [219-225]/interface [220,226] modes (which it does not account for) become preponderant in the signal. Richter et al. were aware of this limitation when they proposed the PCM [180] and Roy et al. simultaneously considered confined bulk modes (PCM description) and surface phonons in CdS/CdSe nanoparticles embedded in glass matrix [223]. The surface modes in ionic crystals become important below $\sim 100 \mathrm{~nm}[227,228]$ but can be predicted using a "Dielectric Continuum Approach" (DCA). Surface Optical modes (SO) are expected at wavenumbers $\bar{v}_{\text {SO }}$ in the intermediate region between the TO and LO modes, which splitting is a consequence of the long range Coulomb interaction. In spherical crystals of Blende type semiconductors [224,227,229]:

$$
\bar{v}_{\mathrm{SO}}=\sqrt{\frac{\frac{\ell+1}{\ell} \bar{v}_{\mathrm{TO}}^{2}+\frac{\varepsilon_{\infty}}{\varepsilon_{\mathrm{M}}} \bar{v}_{\mathrm{LO}}^{2}}{\frac{\ell+1}{\ell}+\frac{\varepsilon_{\infty}}{\varepsilon_{\mathrm{M}}}}}
$$

In Eq. (14), $\ell=1,2,3 \ldots, \varepsilon_{\infty}$ is the high frequency dielectric constant of the semiconductor and $\varepsilon_{M}$ is the frequency-independent dielectric constant of the surrounding medium. The radial dependence of surface modes is in the $\mathrm{r}^{{ }^{1}}$ form and only the $\ell=1$ surface mode (the socalled Fröhlich mode), with its constant amplitude, is thus expected to make a significant contribution. Surface optical modes were similarly described for semiconductors of the Wurtzite type [226] and metals [230]. Surface/Interface modes for the specific geometries of nanocylinders and nanowires [222], spherically capped QDs/Quantum Wells (QWs) [231] and Multiple QWs (MQWs) [232,233] have also been addressed.

iv) the confinement function has limited physical meaning: Most critics formulated against the PCM concerned the arbitrariness of the confinement function. As a matter of fact, the PCM only is a phenomenological tool and even its best advocates acknowledge it can not be expected to fully account for the lineshape when it relies on propagating phonons ("bulk" dispersions curves) to describe confined modes. Alternative and more "physical" descriptions of optical modes confined in nanocrystals were mostly proposed by semiconductor specialists. First, it was predicted [234] and confirmed experimentally for $\mathrm{PbS}$ [235] that $\mathrm{TO}$ and LO modes couple with each other in nanocrystals. Besides, the dipoles generated in polar semiconductors by optical vibrations generate electromagnetic fields and interact with electrons (polarons) or electron-hole pairs (excitons). This so-called Fröhlich interaction is weak in single crystals but leads to a strong resonance of LO and SO modes in confined 
semiconductors: quantum dots, wires and supperlattices [196,220,224,234,236-240]). Pusep et al. tried to adapt the PCM to the electron-LO coupling in doped semiconductors [241] but accurate descriptions require continuous approaches like the one proposed by Roca, TralleroGiner and Cardona [234], later improved by Vasilevskiiy and co-workers [242]. Note that in conducting/coloured nanomaterials (carbon, conducting polymers, etc) and superlattices, a Fano coupling is possible between the continuum of electronic states and the discrete energy levels of the phonons [212,243-246].

\section{$\underline{\text { 3.3.2 } \rightarrow \text { The Elastic Sphere Model (ESM) }}$}

The use of bulk dispersion curves is questionable when there is a lot of reduction in particle size [9,238]. Meyer et al. used Molecular Dynamics Simulations to calculate the nanoparticle Vibrational Density Of States (VDOS) and demonstrated the importance of grain boundaryrelated contributions [247]. An alternative to considering the vibrations as ensuing from a disturbed infinite crystal (what the PCM does) is to adopt a first principle description of vibrations in a free sphere. This problem was theoretically discussed in 1882 by Lamb [248]. A brief description will be given but detailed information can be found elsewhere (with a broad spectrum of notations) [249-257]. Imagine a sphere of radius $R$ with vector $\vec{u}(M, t)$ as the displacement field induced by propagating waves at a given point $(\mathbf{M})$ and for a given time ( $\mathrm{t}$ ). Assuming the sphere is homogeneous (constant density $\rho$ ) and elastic, then elastodynamics theory commands the waves to satisfy the general Navier equation:

$$
\ddot{\mathbf{u}}=\left(\frac{\lambda+2 \mu}{\rho}\right) \nabla(\nabla \cdot \overrightarrow{\mathbf{u}})-\frac{\mu}{\rho} \nabla \times(\nabla \times \overrightarrow{\mathbf{u}})=\mathrm{v}_{\mathrm{L}}^{2} \nabla(\nabla \cdot \overrightarrow{\mathbf{u}})-\mathrm{v}_{\mathrm{T}}^{2} \nabla \times(\nabla \times \overrightarrow{\mathbf{u}})=\left(\mathrm{v}_{\mathrm{L}}^{2}-\mathrm{v}_{\mathrm{T}}^{2}\right) \nabla(\nabla \cdot \overrightarrow{\mathbf{u}})+\mathrm{v}_{\mathrm{T}}^{2} \Delta \overrightarrow{\mathbf{u}}
$$

In Eq. (15), $\ddot{\mathbf{u}}$ represents the second time derivative of vector $\overrightarrow{\mathrm{u}}, \boldsymbol{\lambda}$ and $\boldsymbol{\mu}$ are the so-called Lamé coefficients characterizing the bulk mechanical properties ${ }^{11}$ while $\mathbf{v}_{\mathbf{L}}$ and $\mathbf{v}_{\mathbf{T}}$ are respectively the longitudinal and transverse sound propagation velocities. A general law states that a scalar potential $\phi$ and a 2-component vector potential $\psi$ exist with which $\overrightarrow{\mathrm{u}}$ can be expressed as the sum of one irrotational $\left(\mathbf{u}_{1}\right)$ and two divergence-free ( $\mathbf{u}_{2}$ and $\mathbf{u}_{\mathbf{3}}$ ) components:

$$
\begin{gathered}
\Phi=\frac{1}{\mathrm{~h}} \mathrm{j}_{1}\left(\mathrm{k}_{\mathrm{L}} \mathrm{r}\right) \mathrm{Y}_{1}^{\mathrm{m}}(\theta, \varphi) \mathrm{e}^{-\mathrm{i} \omega \mathrm{t}} ; \mathrm{k}_{\mathrm{L}}=\frac{2 \pi v_{\mathrm{n}}}{\mathrm{v}_{\mathrm{L}}} \\
\psi=\frac{1}{\mathrm{k}} \mathrm{j}_{1}\left(\mathrm{k}_{\mathrm{T}} \mathrm{r}\right) \mathrm{Y}_{1}^{\mathrm{m}}(\theta, \varphi) \mathrm{e}^{-\mathrm{i} \omega \mathrm{t}} ; \mathrm{k}_{\mathrm{T}}=\frac{2 \pi v_{\mathrm{n}}}{\mathrm{v}_{\mathrm{T}}} \\
\mathrm{u}_{1}=\nabla \Phi \\
\mathrm{u}_{2}=\nabla \times \nabla(\overrightarrow{\mathrm{r}} \psi) \\
\mathrm{u}_{3}=\mathrm{k}_{\mathrm{T}} \nabla \times(\overrightarrow{\mathrm{r}} \psi)
\end{gathered}
$$

In Eq. (16) and (17), $\mathrm{j}_{1}$ are spherical Bessel functions of the first kind and $\mathrm{Y}_{1}^{\mathrm{m}}$ are the usual spherical harmonics. The radial quantum number $\mathrm{n}$ indicates the mode order $(\mathrm{n}=0$ for surface modes $[250,258])$ while the orbital quantum number 1 and its $z$-axis component $\mathrm{m}(-1 \leq \mathrm{m} \leq$ $+1)$ define the symmetry of the vibrations and the number of nodal surfaces ${ }^{12} . \phi$ governs

\footnotetext{
${ }^{11}$ They are related to Young's Modulus (E) and Poisson's Ratio $(\sigma)$ via the following relations:$$
\lambda=\frac{\sigma \mathrm{E}}{(1+\sigma)(1-\sigma)} ; \mu=\frac{\mathrm{E}}{2(1+\sigma)}
$$

${ }^{12}$ Duval et al. added a forth parameter -the polarization index p-, to distinguish spheroidal modes from torsional ones [259].
} 
longitudinal (compressive) waves (P-waves in seismology) whereas $\Psi$ is associated with transverse (shear) waves (S-waves in seismology). For convenience, replace the vector base classically used in problems with spherical symmetry (Figure 7-a) by the following one :

$$
\begin{aligned}
\overrightarrow{\mathrm{e}}_{\mathrm{lm}}(\theta, \varphi) & =\mathrm{Y}_{1}^{\mathrm{m}}(\theta, \varphi) \vec{e}_{r} \\
\overrightarrow{\mathrm{n}}_{\mathrm{lm}}(\theta, \varphi) & =\overrightarrow{\mathrm{r}} \nabla \mathrm{Y}_{1}^{\mathrm{m}}(\theta, \varphi) \\
\overrightarrow{\mathrm{m}}_{\mathrm{lm}}(\theta, \varphi) & =\overrightarrow{\mathrm{e}}_{\mathrm{r}} \times \overrightarrow{\mathrm{n}}_{\mathrm{lm}}(\theta, \varphi)
\end{aligned}
$$

In this new base, Eq. (18) to (20) can be rewritten as follows (the prime indicates differentiation with respect to $r)$ :

$$
\begin{gathered}
\mathrm{u}_{1}=\mathrm{j}_{1}^{\prime}\left(\mathrm{k}_{\mathrm{L}} r\right) \overrightarrow{\mathrm{e}}_{\mathrm{lm}}(\theta, \varphi)+\frac{\mathrm{j}_{1}\left(\mathrm{k}_{\mathrm{L}} r\right)}{\mathrm{k}_{\mathrm{L}} r} \overrightarrow{\mathrm{n}}_{\mathrm{lm}}(\theta, \varphi) \\
\mathrm{u}_{2}=1(1+1) \frac{\mathrm{j}_{1}\left(\mathrm{k}_{\mathrm{L}} r\right)}{\mathrm{k}_{\mathrm{L}} r} \overrightarrow{\mathrm{e}}_{1 \mathrm{~m}}(\theta, \varphi)+\left(\frac{\mathrm{j}_{1}\left(\mathrm{k}_{\mathrm{L}} r\right)}{\mathrm{k}_{\mathrm{L}} r}+\mathrm{j}_{1}^{\prime}\left(\mathrm{k}_{\mathrm{L}} r\right)\right) \overrightarrow{\mathrm{n}}_{\mathrm{lm}}(\theta, \varphi) \\
\mathrm{u}_{3}=-\mathrm{j}_{1}\left(\mathrm{k}_{\mathrm{T}} \mathrm{r}\right) \times \overrightarrow{\mathrm{m}}_{\mathrm{lm}}(\theta, \varphi)
\end{gathered}
$$

A distinction is now possible between two families of modes (see Figure 7-b\&c) :

Modes with purely radial displacement $\left(\mathbf{u}_{3}=0\right)$ are called spheroidal modes.

Modes with purely tangential displacement $\left(\mathbf{u}_{1}=\mathbf{u}_{2}=0\right)$, occurring with no volume change, are called torsional modes.

a)

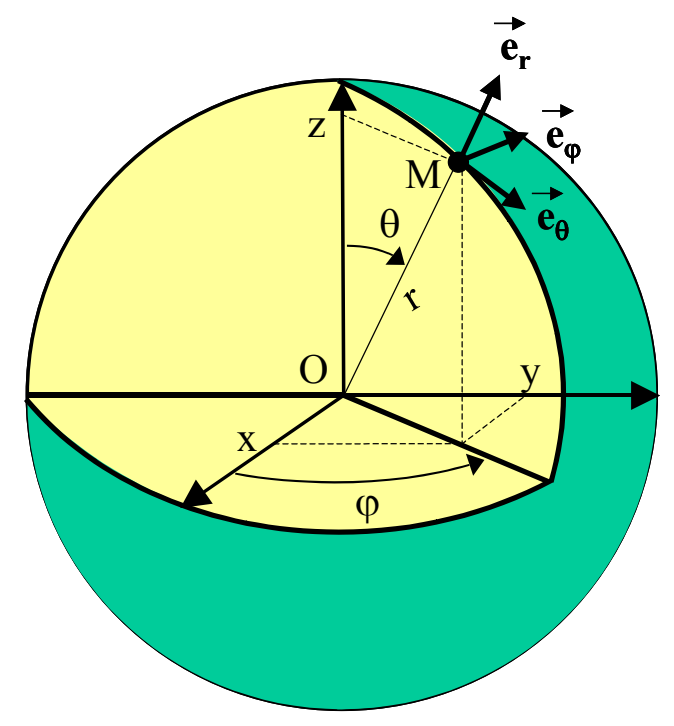

b)

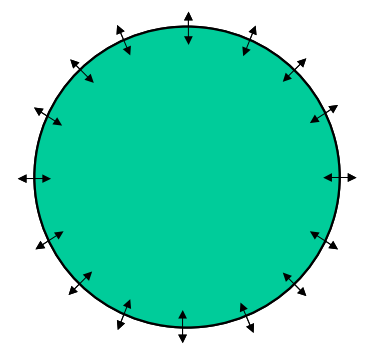

c)

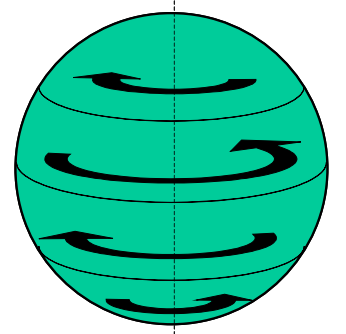

Figure $7:$ a) Description of one nanoparticle in the Elastic Sphere Model (ESM) - b) Oscillation of a free sphere in one spheroidal mode - c) Oscillation of a free sphere in one torsional mode.

Duval was the first to establish the selection rules for the ESM [260]. Since the modes irreducible representations had to coincide with those of the sphere group, he showed that only purely spherical (irreducible representation $\left.\mathrm{D}_{\mathrm{g}}{ }^{(0)}\right)$ and quadrupolar $\left(\mathrm{D}_{\mathrm{g}}{ }^{(2)}\right)$ spheroïdal

\footnotetext{
${ }^{13}$ Duval et al. added a forth parameter -the polarization index p-, to distinguish spheroidal modes from torsional ones [259].
} 
modes are Raman active (while all torsional modes are forbidden) ${ }^{14}$. Their relative intensity is variable but only the first order "surface" ones, those shown in Figure 8, have significant contributions [258,261-263]. Their identification is easy since only $\mathrm{D}_{\mathrm{g}}{ }^{(0)}$ is polarized (parallel polarization). An expression for the stress field can be derived from Equations (24) to (26) and the subsequent application of stress free boundary conditions for $\mathrm{r}=\mathrm{R}$ eventually yields the vibration eigenfrequencies $v_{\mathrm{n}}{ }^{15}$. For $1=0$ spheroidal modes, a good approximation of $v_{\mathrm{n}}$ is given by [257]:

$$
v_{\mathrm{n}}=\frac{\mathrm{v}_{\mathrm{L}}}{\mathrm{R}}\left((\mathrm{n}+1) \pi-\arcsin \frac{4}{(\mathrm{n}+1) \pi}\left(\frac{\mathrm{v}_{\mathrm{T}}}{\mathrm{v}_{\mathrm{L}}}\right)^{2}\right)
$$

No analytic solutions can be obtained for the other modes, which have to be calculated numerically, but the wavenumbers are always proportional to $\mathrm{R}^{-1}$ (see Figure 8) :

$$
\begin{aligned}
\mathrm{R} & =\frac{\mathrm{A} \cdot \mathrm{v}_{\mathrm{L}}}{\mathrm{c} \cdot \mathrm{v}_{\mathrm{D}_{\mathrm{g}}^{(0)}}} \\
\mathrm{R} & =\frac{\mathrm{A}^{\prime} \cdot \mathrm{v}_{\mathrm{T}}}{\mathrm{c} \cdot \mathrm{v}_{\mathrm{D}_{\mathrm{g}}^{(2)}}}
\end{aligned}
$$

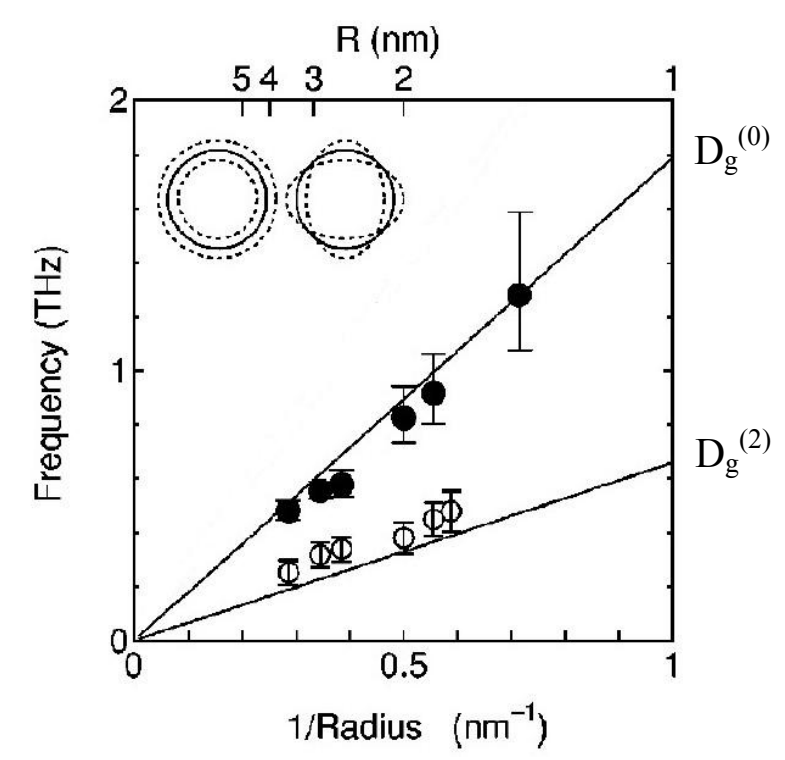

Figure 8 : Size dependence of the $\mathrm{D}_{\mathrm{g}}{ }^{(0)}$ and $\mathrm{D}_{\mathrm{g}}{ }^{(2)}$ modes in PbSe quantum dots. Reprinted with permission from M. Ikezawa et al., Phys. Rev. B64, 201315, 2001. Copyright 2001 by the American Physical Society.

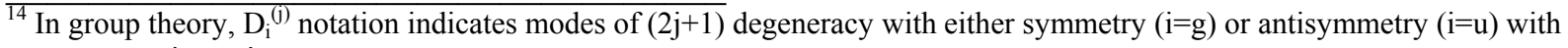
respect to an inversion centre.

${ }^{15}$ Some authors added electromagnetic continuity as a grain boundary condition [234,242].
} 
In Eq. (28) and (29), $\mathrm{c}$ is the light speed in vacuum while $\mathrm{A}$ and $\mathrm{A}^{\prime}$ are dimensionless constants. These equations were used to measure the radius of silver clusters in ancient and advanced materials ${ }^{16}$ [264-267] or particles size in cordierite [262], $\mathrm{TiO}_{2}$ [268-270], silicon $[271,272], \mathrm{PbSe}$ [273], etc. Note that the scattering efficiency of the particles scales like $v^{-3}$ [261]. Consequently, directly applying Eq. (28) and (29) when size is distributed will lead to an overestimation of $\mathrm{R}$ average. Besides, the ESM assumes the nanoparticles are homogeneous and only modes with $\lambda$ much greater than the interatomic distance, mostly the low energy fundamental and $1^{\text {st }}$ harmonic modes, are described [259]. On account of that restriction, the ESM is often referred to as a predicting model for "confined acoustic modes", although optic phonons were occasionally studied using the same model [274].

Just like for the PCM, the use of the ESM for grain size determination comes with certain approximations. As is, the model strictly applies to equal size, "free-standing", isotropic spheres. The triple degeneracy of $\mathrm{D}_{\mathrm{g}}{ }^{(2)}$ mode is lifted for ellipsoidal particles $[260,275]$ and Hernandez-Rosas et al. recently proposed a generalised model for oblate spheroids [276]. Like for the PCM, it is also possible to include the size distribution [218,265,277,278] and the problem of anisotropic materials was discussed by Murray et al. [279]. It had been previously dealt with by Fujii et al. who tried calculations based on the longitudinal and transversal sound velocities for the main crystallographic directions of silicon but they found no agreement with their experimental results [271].

It has been assumed for a long time that the ESM held in matrices with Lamé coefficients sufficiently different from those of the nanospheres [280,281], but the presence of a matrix also activates a torsional surface mode $(l=1)$ which eventually dominates the spectra [260,280,281]. Moreover, some couplings of the Raman active modes with matrix vibrations can be expected [250-252,256,277,279-282], knowing that the smaller the nanoparticles, the stronger the coupling [251,257]. They were modelled by a "Complex Frequency Method" (CFM) $[259,279]$. If the nanoparticles are in relatively high concentration, which will surely be the case in active devices like QDs networks, a collective Fröhlich Mode will also contribute to the Raman spectrum [283].

\section{$\underline{3.3 .3 \rightarrow \text { Validity Domains of the PCM and the ESM. }}$}

Figure 9 illustrates the different approaches to the RS of nanoparticles. In grains much larger than the wavelength, phonons propagate almost in the same way as in perfect "infinite" crystals. The only perturbation then comes from vibrations involving the outer atomic layers, which do not meet the bulk conditions $[37,80,196]$. The structural transition between the surface and the bulk of each grain obviously is progressive but let us assume, for simplicity, that there is an homogeneous surface shell of definite thickness t. Figure 9-a then shows how the skin to bulk volume ratio reaches a critical value for $D / t \sim 5$, where $D$ is the grain diameter. When the grain size further decreases, the confinement of optic modes begins and the PCM starts applying. Soon, the relative proportion of "non-bulk" skin and surface modes increases and, below a certain size, the very notion of collective vibrations disappears. As a principle, the ESM must then be considered but there is no clear-cut grain dimension for which one

\footnotetext{
${ }^{16}$ Most authors only considered $\mathrm{Ag}^{0}$ [264] but clusters integrating $\mathrm{Ag}^{+}$ions are rather expected in a iono-covalent matrix (there are $\mathrm{Ag}^{+}$ions at the surface of the clusters formed in oxides fired at high temperatures [4]). Analysis with different excitations should reduce $\mathrm{Ag}^{+}$contribution through metallic silver exaltation.
} 
should switch from the PCM to the ESM. They do not necessarily exclude each other and, on occasion, were even used simultaneously [204,255,284,285].

i) The PCM should be applicable between the grain size at which "bulk" dispersion curves lose all significance $(\sim 5 \mathrm{~nm}[206,238])$ and a high-end value where confinement has no effect on the spectrum, which will obviously depend on the mode dispersion around BZc $[40,193,197,285]$. Note that surface modes add a significant contribution for grain sizes up to $8 \mathrm{~nm}$ in $\mathrm{SnO}_{2}$ [286] and even $20 \mathrm{~nm}$ in $\mathrm{ZrO}_{2}$ [287].

ii) The typical grain size below which nanocrystals can not be assumed homogeneous and the ESM therefore no longer applies [250] is between 1 [266] and 5 [259,285,288] nanometers, which is in good agreement with the $\sim 40$ interatomic distances predicted by Wittmer et al. [289]. Note if particles with a size slightly above this limit are embedded in a glass phase, the "so-called" boson peak of the matrix (see $\S 4.1$ ) may hide the free vibrations described by the ESM, unless wave-guided spectroscopy is used [266]. There is no theoretical upper limit to ESM applicability but the low frequency modes gradually enter the Rayleigh wing (quasielastic diffusion; see $\S 2.2$ ) as size go increasing and become unusable for a value that depends on both the material and the experimental resolution of the spectrometer $[255,290]$.

a)
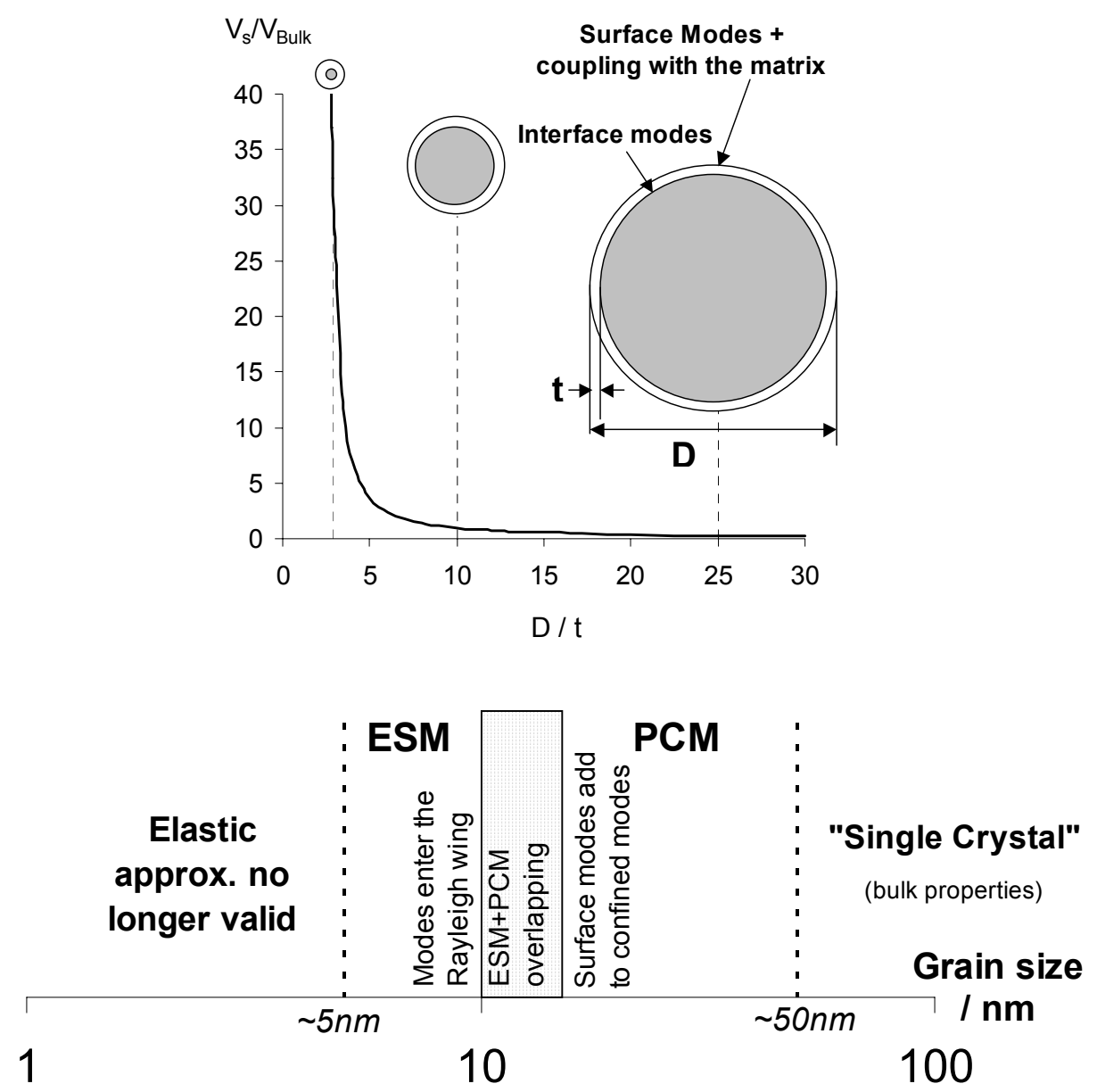

Figure 9 : a) The "surface to bulk" volume ratio in nanospheres of diameter D and constant "skin thickness" t (see text) - b) Applicability range of the different descriptions for the vibrations of spherical crystals (ESM = Elastic Sphere Model $; \mathrm{PCM}=$ Phonon Confinement Model). The grain size values are indicative and actual limits depend on the material. 
One should not forget that peak altering effects like internal stress ${ }^{17}$ [9,185,187,216,217,219,292], growth directionality [81], non-stoichiometry [186], local heating [190,222,293], couplings or defect-induced diffusions [293] may add to the size effect on Raman spectra of nanomaterials. The stress will act on the position of the peaks [187], defects (non-stoichiometry for instance) will widen them [186,187,190] and temperature will act on both position and width. Falkovsky and Camassel wrote a short review on the contribution of all kinds of defects to optical peaks widening [294].

\section{SELECTED CASE STUDIES}

\subsection{The Structural Variety of Glass Ceramics}

a)

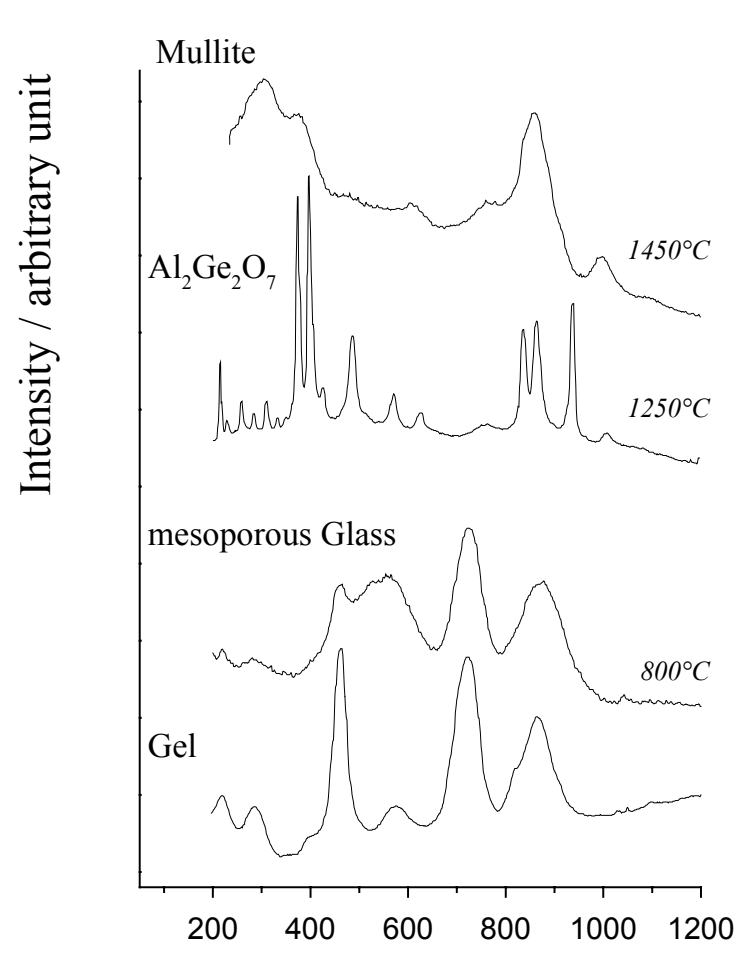

b)
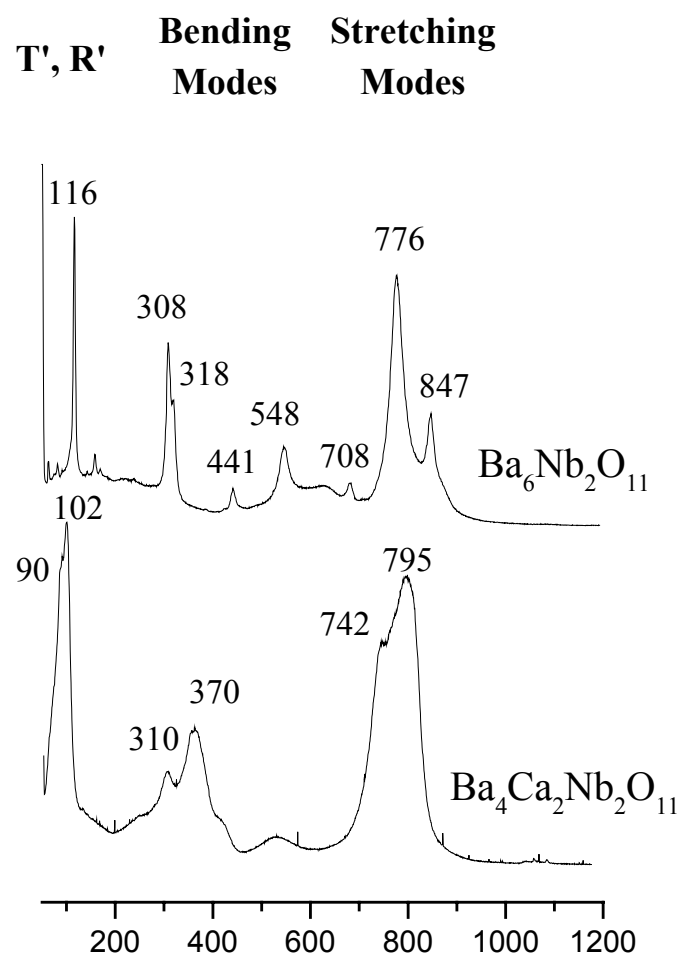

Wavenumber $/ \mathrm{cm}^{-1}$

Figure 10 : a) Germanium mullite in different physical states (Adapted from D. Michel et al., J. Europ. Ceram. Soc., 16, pp 161-168, 1996) - b) Perovskite-related barium niobiates (Adapted from Ph. Colomban et al., Sol. State Ionics, 145, pp 339-347, 2001).

Ceramics and glass-ceramics obtained from liquid precursors (molten glass and salts, solutions, gels, organic precursors [1,295-297]) form a large range of nanophased materials. The transition from the liquid to the solid state "freezes" a local steric disorder and thermal annealing is necessary for long distance Coulombian interactions to stabilize the

\footnotetext{
${ }^{17}$ Residual stresses are unavoidable, even in free particles (the reduction of the surface atoms coordination number reduces the bond lengths [291]). They were investigated in detail by Gomonnai et al. [219] and Sirenko et al. [9].
} 
thermodynamically stable crystalline phase. In these materials, strongly covalent species like the $\mathrm{SiO}_{4}{ }^{4-}$ or $\mathrm{PO}_{4}{ }^{3-}$ tetrahedra often "see" a site symmetry different from the average one predicted by crystallographic study [297,298]. For instance, Figure 10-a compares the Raman spectrum of a $2 \mathrm{GeO}_{2}-\mathrm{Al}_{2} \mathrm{O}_{3} \sim 2 \mathrm{H}_{2} \mathrm{O}$ gel prepared through alkoxides hydrolysispolycondensation with those of the phases obtained after thermal treatment up to $1450^{\circ} \mathrm{C}$ [299]. The intermediate $\mathrm{Al}_{2} \mathrm{Ge}_{2} \mathrm{O}_{7}$ phase (stable from 1250 to $1300^{\circ} \mathrm{C}$ ) has low symmetry monoclinic structure but fine low frequency Raman peaks, characteristic of an ordered compound, while the spectrum of crystalline mullite is as broad as that of the glass, in spite of a higher symmetry orthorhombic structure. This relates to an orientational disorder induced by oxygen vacancies in the $\mathrm{AlO}_{4}$ tetrahedra. Moreover, since the occurrence of broadening indicates distorted entities, it is obvious from the figure that $\mathrm{GeO}_{4}$ tetrahedra as well as $\mathrm{GeO}_{6}$ octahedra are more distorted in the mesoporous glass than in the gel phase [299]. In the example of the perovskite-related niobiates from Figure 10-b, Nb-O modes around $800 \mathrm{~cm}^{-1}$ split because of oxygen vacancies in the $\mathrm{NbO}_{6}$ octahedra. The ( $\left.\mathrm{T}^{\prime}, \mathrm{R}^{\prime}\right)$ modes involving the $\mathrm{Ba}$ atoms located at the corners of the unit-cell are not affected by these vacancies in $\mathrm{Ba}_{6} \mathrm{Nb}_{2} \mathrm{O}_{11}$ (hence the fine peaks) but split in $\mathrm{Ba}_{4} \mathrm{Ca}_{2} \mathrm{Nb}_{2} \mathrm{O}_{11}$ because chemical and mass decoupling isolate $\mathrm{Ba}$ and $\mathrm{Ca}$ sub-lattices [300]. The same kind of vibrational decoupling with defects has been observed for many silicates [297,298,301].

a)

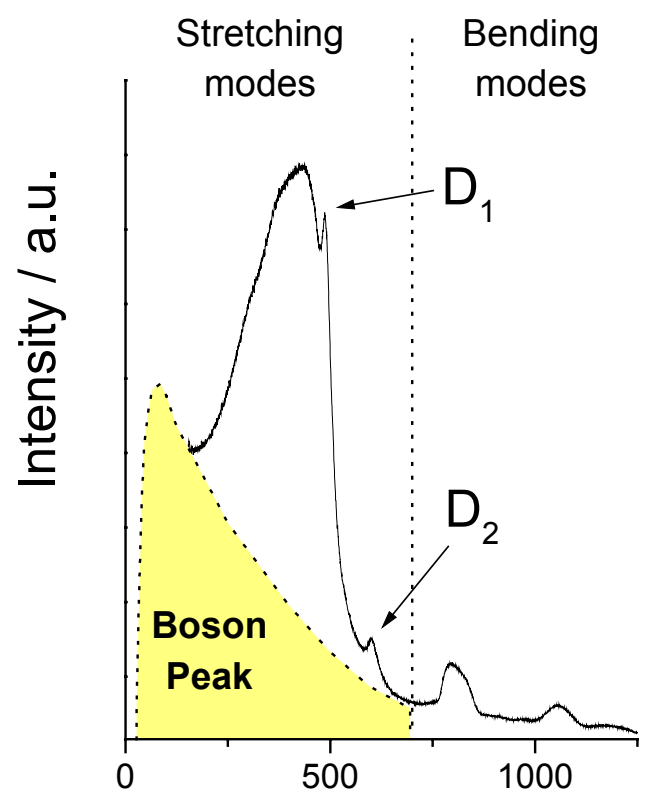

b)

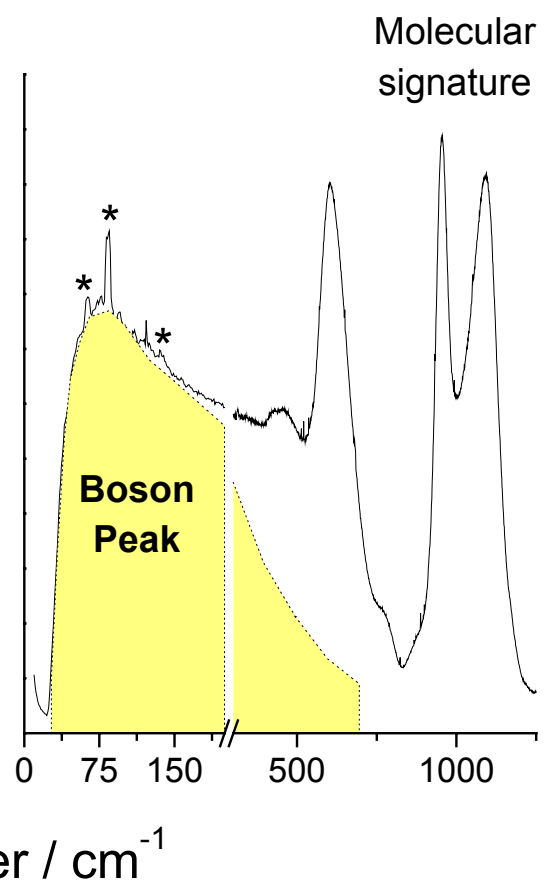

Figure 11 : a) Raman spectrum of pure glassy silica (macro-configuration, $\lambda=406 \mathrm{~nm}$ ) $-\mathbf{b}$ ) Raman spectrum of a highly depolymerised potassium-rich calcium silicate stained glass (macro-configuration, $\lambda=413 \mathrm{~nm}$; stars indicate plasma lines from the laser; $\mathrm{D}_{1}$ and $\mathrm{D}_{2}:$ see text).

Two silicate spectra are shown in Figure 11-a\&b. Most types of glass contain nanometric crystallites and the spectrum of silicates $\left(\mathrm{SiO}_{2}\right.$ tetrahedral network) is intermediate between that of a disordered network and the superposition of contributions from definite vibrational 
entities : tetrahedra rings in vitreous silica (the narrow defect bands in Figure 11-a originate from the vibrations of 3-fold $\left(\mathrm{D}_{2}\right)$ and 4-fold $\left(\mathrm{D}_{1}\right)$ rings [302-305]) and complex arrangements of $\mathrm{SiO}_{4}$ tetrahedra in alkali/earth-alkali silicates [302]. The deformation $\left(\delta_{\mathrm{Si}-\mathrm{O}-\mathrm{Si}} \sim 500 \mathrm{~cm}^{-1}\right)$ and elongation $\left(v_{\mathrm{Si}-\mathrm{O}} \sim 800-1200 \mathrm{~cm}^{-1}\right)$ modes depend on the connectivity of the $\mathrm{SiO}_{4}$ tetrahedra and can be fitted with components called $Q_{n}$ and $Q_{n}{ }^{\prime}$, respectively ( $n$ represents the number of Si-O-Si bridges per tetrahedron) [306-308]. The position and area of these components then constitute characteristic parameters of silicate glass nanostructures and can be used for garnering additional information such as the original composition or the sintering temperature [306,307].

The most specific part of glass spectra is a contribution peaking at $\sim 50-100 \mathrm{~cm}^{-1}$, which is called the Boson Peak (BP) because its intensity obeys Bose-Einstein distribution. Since F function from Eq. (N3) never descends to zero in amorphous materials, their $\operatorname{VDOS} G(\bar{v})$ is fully active and Raman intensity is given by [309 $]^{18}$ :

$$
\mathrm{I}(\bar{v})=\frac{\mathrm{n}^{\mathrm{B}}(\bar{v})+1}{\mathrm{c} \bar{v}} \mathrm{C}(\bar{v}) \mathrm{G}(\bar{v})
$$

The BP mostly originates from the probe-excitation coupling function $\mathrm{C}(\bar{v})$, the reason why it best shows on $\mathrm{I} /\left[\bar{v}\left(\mathrm{n}^{\mathrm{B}}(\bar{v})+1\right)\right]$ "reduced intensity" curves ${ }^{19}$. There is an ongoing debate to ascertain whether the extra vibrations from the BP correspond to propagating or localized modes but all interpretations make the BP a way of characterizing glass intermediate range order at the nanometer scale:

i) Some teams believe that collective acoustic waves are possible in glass, even at very low frequencies, but that the transverse acoustic branch is flattened [310-312]. Yet, $C(\bar{v})$ is expected to scale as $\bar{v}^{2}$ for slightly distorted plane-wave vibrations [313] when Surotsev and Sokolov demonstrated experimentally that glasses rather exhibit the following behavior $[314]^{20}$ :

$$
C(\bar{v})=A \times\left(\frac{\bar{v}}{\bar{v}_{B P}}+B\right)
$$

ii) Duval et al. explain the change in the VDOS by elastic constants fluctuations in the random non-continuous structure of the glass $[319]^{21}$. In this scheme, amorphous regions whose only difference lies in the strength of their bonds coexist and the denser ones (referred to as "blobs") confine the vibrations. Surotsev recently improved the model by taking into account the contact between the "blobs" instead of considering a separation by soft matter [321]. This refinement introduces some diffusive character to the BP.

\footnotetext{
${ }^{18}$ Note that the spectrum does not strictly identify with the VDOS. Moreover, by comparison with Eq. (N2), C( $\left.\bar{v}\right) \mathrm{G}(\bar{v})$ is analogous to the conductivity of the material. Quasi-Elastic and Inelastic Neutron Scattering also give insight into the frequency dependence of conductivity through the $\mathrm{P}(\omega)=\omega \times \sigma(\omega)$ function (Chap. 21, 23, 25 and 30 in Ref. [54]).

${ }^{19}$ If one assumes, following the standard Debye model for acoustic branches, that $G(\bar{v})$ is proportional to $\bar{v}^{2}$, then the reduced intensity is expected to be proportional to $C(\bar{v})$ (see Eq. (30)).

${ }^{20}$ Slopes different from 2 on Log-Log scale plots of $C(\bar{V})$ were tentatively explained by the fractal nature of the tetrahedral network in gel-derived silica glass (silica aerogels) [313,315-318].

${ }^{21}$ The once popular model from Martin and Brenig attributed the activation of Raman acoustic modes to density fluctuations modelled with a phenomenological Gaussian function [320].
} 
iii) The Soft Potential Model (SPM) considers the extra vibrations as Quazi-Localized Vibrations (QLVs) that resonate with sonic waves. These QLVs are vibrations created by disorder (or, possibly, low lying optical modes) that concern all atoms but with only a small group of them vibrating with significant amplitude. The model was recently improved by Gurevich et al. who introduced anharmonic effects [322].

\subsection{Analysis of Localized Species and Sublattices}

Features from $\mu$ RS can be specific to localized vibrations of weakly bonded or light species like, for instance, $\mathrm{S}_{2}{ }^{-}$ions entrapped in Lapis Lazuli [61]. In Bi2201 and Bi2212 bismuth cuprates, some modes are characteristic of the atom to which oxygen is bonded [323] while the activation of forbidden modes is related to the order of the mobile oxygens [324,325]. Similarly, Figure 12 shows how polyaniline polymerisation produces little spectral modifications [88] while the orientational medium range disorder induced by the grafting (for protonic conduction) of sulfonic groups on the PTFE framework of Nafion ${ }^{\mathrm{TM}}$ polymer membranes produces obvious effects below $400 \mathrm{~cm}^{-1}$ [326].

a)

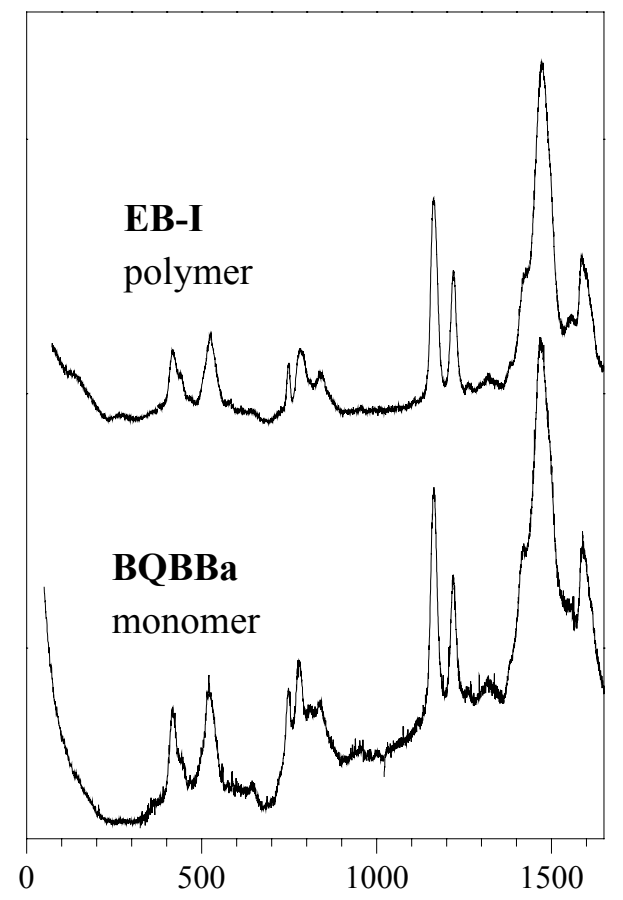

b)

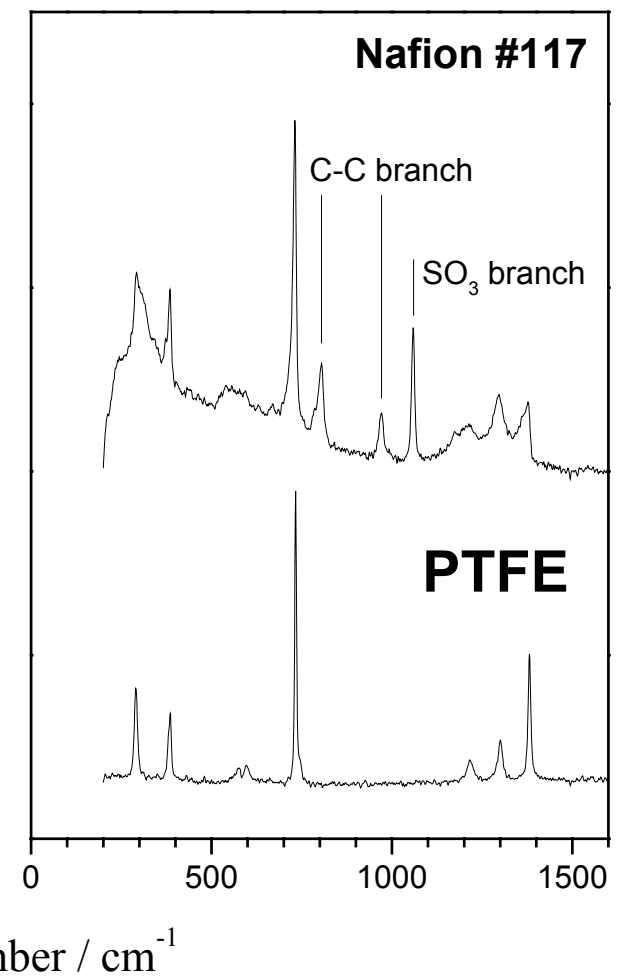

Figure 12 : a) Comparison between the spectra from polyaniline precursor monomer $(1 \sim 1$ $\mathrm{nm}$ ) and polyaniline in one emeraldine base form $(1 \sim 200 \mathrm{~nm}$ ) (Adapted from S. Folch et al.,

Macromol., 32, 3080, 1999) - b) Comparison of PTFE and Nafion (PTFE-grafted with sulfonic groups) Raman spectra (Adapted from A. Gruger et al., Vibr. Spectr., 26, 215, 2001).

In fact, $\mu R S$ is very sensitive to sublattices in those many structures built with vibrationally independent species. Whenever the sublattices have chemical independence (e.g. ionocovalent silicate or phosphate frameworks hosting alkali ions), they give independent Raman 
contributions. If these contributions are taken apart by mass discrepancy, defects from either sublattice, for instance the cation and oxygen sublattices of perovskites, can be analysed separately [300,327,328]. The short distance cationic order in $\mathrm{ZrTiO}_{4}[92,191]$ has also been investigated by RS but one of the best examples of sublattice discrimination remains ionic conductors $^{22}$. For instance, Figure 13 shows how a good energetic gap makes it possible to separate the short range ordered domains of conducting ions in $\beta$-alumina (a superionic solid selected in the seventies as a potential electrolyte): $\mathrm{Ag}^{+}$sub-lattice melts around $200 \mathrm{~K}$ whereas alumina host lattice is stable up to $2000 \mathrm{~K}$ [56,329,330]. Because of their light weight and high polarisability, protonic species exhibit particularly interesting dynamics, their vibrations being often independent from the host lattice (modes are localized and weakly coupled with the others) [54,331].

a)

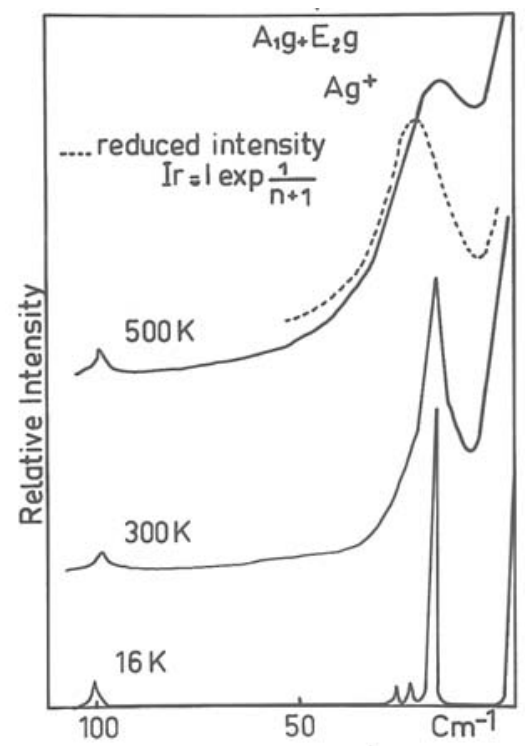

b)

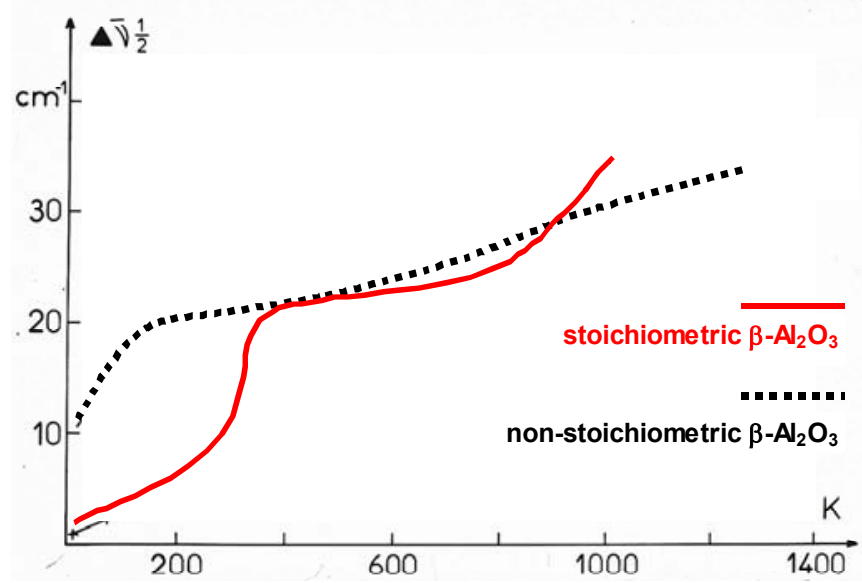

Figure 13 (Adapted from Ph. Colomban et al., J. Chem. Phys., 75[3], pp 1388-1399, 1981) a) The $\sim 25 \mathrm{~cm}^{-1}$ peak of $\beta$-alumina is characteristic of the conducting short range ordered $\mathrm{Ag}^{+}$ bi-dimensional sub-lattice - b) The half-width of the peak makes it possible to identify the melting temperature of the sub-lattice.

\subsection{Carbon Allotropy}

The technique of $\mu \mathrm{RS}$ has been widely used to study carbon allotropes (diamond, graphite, fullerenes, nanotubes ...). It is indeed one of the few techniques sensitive to the full range of structural states present in this class of materials, from perfectly crystalline to amorphous. The common crystalline phases of carbon yield very simple spectra: diamond ( $\mathrm{sp}^{3}$ hybridisation) peaks at $1332 \mathrm{~cm}^{-1}$ (single mode of $\mathrm{T}_{2 \mathrm{~g}}$ symmetry) whereas graphite ( $\mathrm{sp}^{2}$ hybridisation) has doubly degenerate $E_{2 g}$ modes at 42 and $1582 \mathrm{~cm}^{-1}$. The latter is referred to as $G$ band and corresponds to vibrations in the graphene planes (which crystalline quality can be measured by the width of $G$ band [169]) whereas the former corresponds to weak interplanar Van der Waals interactions (the reason for its low energy). Two additional modes appear whenever flaws are created, grain size is reduced or graphene planes are curved [169] (Figure 14-a).

\footnotetext{
$\overline{22}$ In ionic conductors, mobile ions are weakly bonded, which will add to mass contrast in distributing the vibrational energies
} 
These modes are called D and D' (the letter stands for "disorder"). D' results from the splitting of $\mathrm{G}$ band and peaks around $1620 \mathrm{~cm}^{-1}$, at the value where the dispersion curve of graphite is the flattest. The intensity ratio of $\mathrm{G}$ to $\mathrm{D}$ ' depends on the proportion of distorted graphene planes [332]. Assuming Raman scattering efficiencies $\sigma$ are system-independent, then the number $\mathrm{n}$ of consecutive graphene planes should obey the following law [332]:

$$
\frac{\mathrm{I}_{\mathrm{G}}}{\mathrm{I}_{\mathrm{D}^{\prime}}}=\left(\frac{\mathrm{n}-2}{2}\right) \frac{\sigma_{\mathrm{G}}}{\sigma_{\mathrm{D}^{\prime}}}
$$

The interpretation of $\mathrm{D}$ band has been much debated. Despite its proximity to the diamond peak, D is derived from graphite (the very low efficiency of diamond scattering would otherwise make it much weaker). In 2000, Ferrari and Robertson suggested that D band resulted from a resonant enhancement of the modes from graphite dispersion curves having the same wavevector $\mathrm{k}$ as the exciting photons [189]. This assignment provided an explanation for some peculiar features of $\mathrm{D}$ band, such as the excitation dependence of its wavenumber (Figure 14-a), but remained unsatisfying. Thomsen et al. showed that an unusually enhanced double resonance mechanism was at play for D band [333].

a)

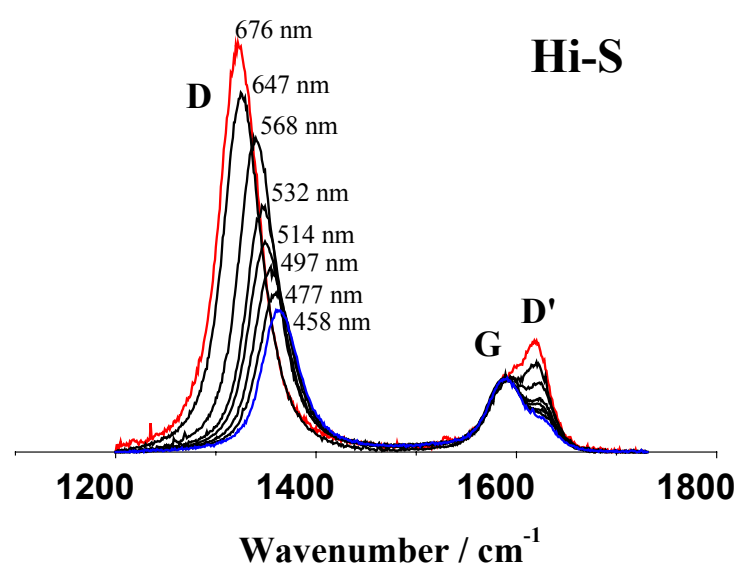

b)

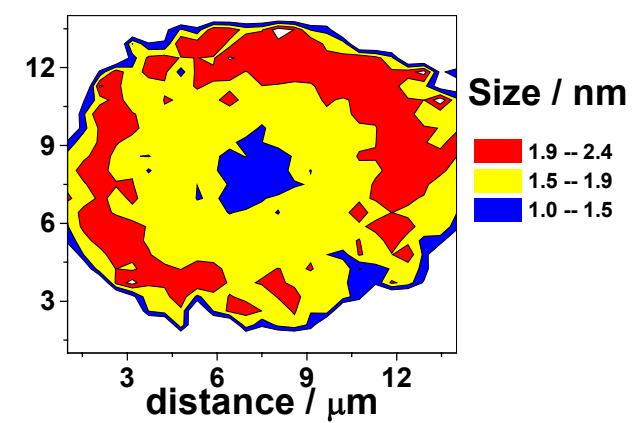

Figure $14:$ a) Raman spectra of the carbon nanoprecipitates in Hi-S Nicalon SiC fibres (Nippon Carbon) as a function of the laser excitation wavelength $\lambda$ (Adapted from G.

Gouadec et al., Comp. Sci. Techn., 62, pp 505-511, 2002) - b) Tuinstra and Konig's calibration (Eq. (33)) was used to build a 2D "map" of carbon grain size in a SA3 SiC fibre (Ube Ind.). This is called "smart mapping" [39,213,215,334]. Reproduced with permission from "Raman Analyses and Smart Imaging of Nanophases and Nanosized Materials", Ph. Colomban, Spectroscopy Europe, 15[6], pp 8-16, 2003. Copyright John Wiley \& Sons Ltd.

Whatever the exact nature of D band, it was soon shown that it depended on carbon grain size, a feature for which an empirical law was proposed in 1970 by Tuinstra and Koenig $[200]^{23}$ :

$$
\frac{\mathrm{I}_{\mathrm{D}^{1350 \mathrm{~cm}}-1}}{\mathrm{I}_{\mathrm{G}^{1580 \mathrm{~cm}^{-1}}}}=\frac{\mathrm{C}(\lambda)}{\mathrm{L}_{\mathrm{g}}(\mathrm{nm})} \quad / \quad \mathrm{C}(\lambda=514.5 \mathrm{~nm})=44
$$

\footnotetext{
${ }^{23}$ This calibration is not valid below $2 \mathrm{~nm}[189]$.
} 
The so-called "Tuinstra and Konig" calibration has been used extensively for the "qualitative" control of carbon structural transformations, mostly in amorphous carbon $[335,336]$ and Diamond-Like Carbon (DLC) [337-343] films but also on radial cross sections in carbon micro-rods [344] and electrodes [345]. A direct proportionality between $\mathrm{I}_{\mathrm{D}} / \mathrm{I}_{\mathrm{G}}$ ratio and the $\mathrm{sp}^{3}$ content, as obtained from EELS experiments, has been reported in amorphous carbon films [346].

In 1985, Kroto et al. were the first to find experimental evidence of crystalline forms of carbon other than diamond and graphite [347]. These corresponded to an assembling of pentagonal, hexagonal and heptagonal carbon rings to form spheroids (fullerenes) [348]. Crystallization by closing of rolled graphene planes is also possible, in which case nanotubes (NTs) are formed [349]. These NTs have enormous application potential as they are among the hardest known material and are able to conduct single charges unidirectionally [350]. Figure 15-a shows how one $\{n, m\}$ couple of integers is sufficient to fully characterize a NT. This tube is obtained by the rolling of the chiral vector $\vec{c}=n \vec{a}_{1}+m \vec{a}_{2}$ in a graphene plane and its diameter $\mathrm{d}_{\mathrm{NT}}$ and chiral angle $\theta$ are simply given by:

$$
\begin{aligned}
\mathrm{d}_{\mathrm{NT}} & =\frac{\|\overrightarrow{\mathrm{c}}\|}{\pi}=\frac{1_{\mathrm{b}} \sqrt{3} \sqrt{\mathrm{n}^{2}+\mathrm{m}^{2}+\mathrm{nm}}}{\pi} \\
\theta & =\arcsin \left(\frac{\mathrm{m} \sqrt{3}}{2 \sqrt{\mathrm{n}^{2}+\mathrm{m}^{2}+\mathrm{nm}}}\right)
\end{aligned}
$$

Figure 15-b shows the Raman spectra of some Double Walled CNTs (DWCNTs). The bond angles and lengths no longer correspond to a perfect $\mathrm{sp}^{2}$ hybridisation but Raman modes can still be derived from graphite dispersion curves [351,352]. Eklund et al. showed G wavenumber remains at $1581 \mathrm{~cm}^{-1}$ in NTs with radii above $15 \mathrm{~nm}$ but drops to $1465 \mathrm{~cm}^{-1}$ below [353].

a)

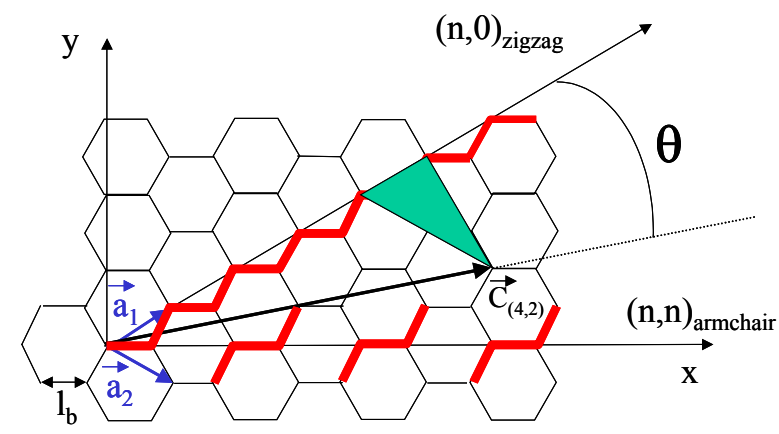

b)

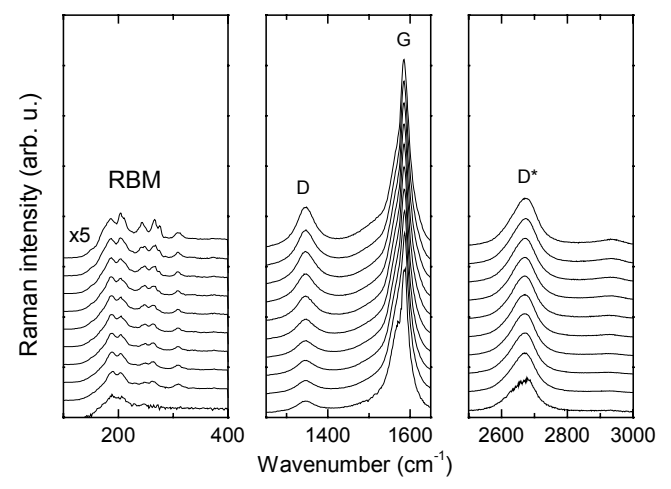

Figure 15 : a) Nanotubes can be described by the rolling of a chiral vector $\vec{c}=n \vec{a}_{1}+m \vec{a}_{2}$ in a graphene plane - b) Raman spectra of Double Wall Carbon Nanotubes (DWCNTs) filled with $\mathrm{PbI}_{2}$. Reprinted with permission from Puech et al., Phys. Rev. B72, 155436, 2005. Copyright 2005 by the American Physical Society. 
It should be noted that the Radial Breathing Mode (RBM) shown in Figure 15-b can be used to measure $\mathrm{d}_{\mathrm{NT}}$ accurately [354]. Based on a zone folding model, Eklund et al. [353] predicted the relationship between $\bar{v}_{\mathrm{RBM}}$ and $\mathrm{d}_{\mathrm{NT}}$, which was calibrated afterwards [355-358]:

$$
\mathrm{d}_{\mathrm{NT}}(\mathrm{nm})=\frac{223.75}{\bar{v}_{\mathrm{RBM}}\left(\mathrm{cm}^{-1}\right)}
$$

NTs with different $\{n, m\}$ may be similar in diameter but they will resonate for different laser energies [359,360]. These energies correspond to interband transitions between the valence and conduction band at Van Hove singularities. The relationship has been established and $\{n, m\}$ can then be precisely found on the basis of $\mu \mathrm{RS}$ [361]. This is interesting because $\{\mathrm{n}, \mathrm{m}\}$ is determinant for many properties. For instance, nanotubes with $|\mathrm{n}-\mathrm{m}|=3 \mathrm{q}$ (q integer) are metallic whereas those for which $|\mathrm{n}-\mathrm{m}|=3 \mathrm{q} \pm 1$ are semiconductors [362]. The behaviour of the D [363,364], G [365] or $D^{* 24}$ [364] bands has also been studied extensively. References [351] and [352] provide thorough descriptions of the RS of CNTs.

\subsection{Nanophased SiC Fibres}

SiC fibres, which are among the most stable inorganic fibres, are mostly produced by the 3D reticulation of a polymeric precursor ${ }^{25}$. Their nano-crystalline structure offers the best compromise between a good homogeneity (a characteristics of the amorphous state) and a high bond density (a characteristics of the crystalline state). Their very smooth surface and the lack of defects explain tensile strengths $\sigma_{\mathrm{r}}$ as high as $3 \mathrm{GPa}$. These fibres are designed to reinforce composites working at very high temperatures $\left(\sim 1200-1400^{\circ} \mathrm{C}\right)$ in oxidizing and corrosive atmospheres (carbon fibres oxidize at $500^{\circ} \mathrm{C}$ whereas alumina, zirconia and mullite fibres properties degrade above $1100-1200^{\circ} \mathrm{C}$ [366] as they crystallise).

Micro-Raman spectroscopy is particularly well suited to study $\mathrm{SiC}$ fibres because it gives a good signal for both $\mathrm{SiC}$ and the secondary carbon phase. Figure 16-a illustrates this fact with $\mathrm{SiC}$ decarburation in NLM fibres submitted to alkaline corrosion. First, the different forms of partially oxidised or nitrided carbon can be identified [23]. As for SiC spectrum, it mainly consists of peaks around 795 and $970 \mathrm{~cm}^{-1}$. They correspond respectively to the transverse optical ( $\mathrm{TO}_{1}$ and $\mathrm{TO}_{2}$; degenerate) and longitudinal optical (LO) modes of $\beta$-SiC ${ }^{26}$, which $\mathrm{X}$ ray diffraction identified as the phase present in Nicalon (NLM, Hi \& Hi-S) [370], SA [371] and Sylramic [372] fibres. Additional bands correspond to SiC polytypism (see Figure 17: Brillouin Zone folding generates satellite lines on the low energy side of TO and LO bands [213,214,373-378]), amorphous SiC (broad symmetric background) and asymmetric VDOS projections when "random" faulting in the stacking sequence folds BZ in countless ways [373].

\footnotetext{
${ }^{24} \mathrm{D} *$ is the overtone of D-band. It is alternatively referred to as $\mathrm{G}^{\prime}$ by analogy with a second order band found in disordered graphite (see Figure 21).

${ }_{25}$ The main alternative is Chemical Vapour Deposition (CVD) of SiC on a carbon fibre core (SCS-6 fibre from Textron).

${ }^{26} \mathrm{In} \mathrm{SiC}$ structures, Si and C "bilayers" are referred to as "h" if they are deduced from the underlying bilayer by a simple translation. However, if an additional $180^{\circ}$ rotation (around the Si-C bonds linking the bilayers) is required for superposition, then bilayers are referred to as " $k$ ". Each periodic stacking sequence defines a polytype which is classified according to Ramsdell notation by first giving the number $\mathrm{N}$ of bilayers in the stacking cell and then the cell type [367-369]. The "all $\mathrm{k}$ " polytype ( $\beta$-SiC phase) is the $3 \mathrm{C}$ (cubic) polytype. All other polytypes ( $\alpha$-SiC phase) are either NH (hexagonal) or NR (rhombohedral).
} 
a)

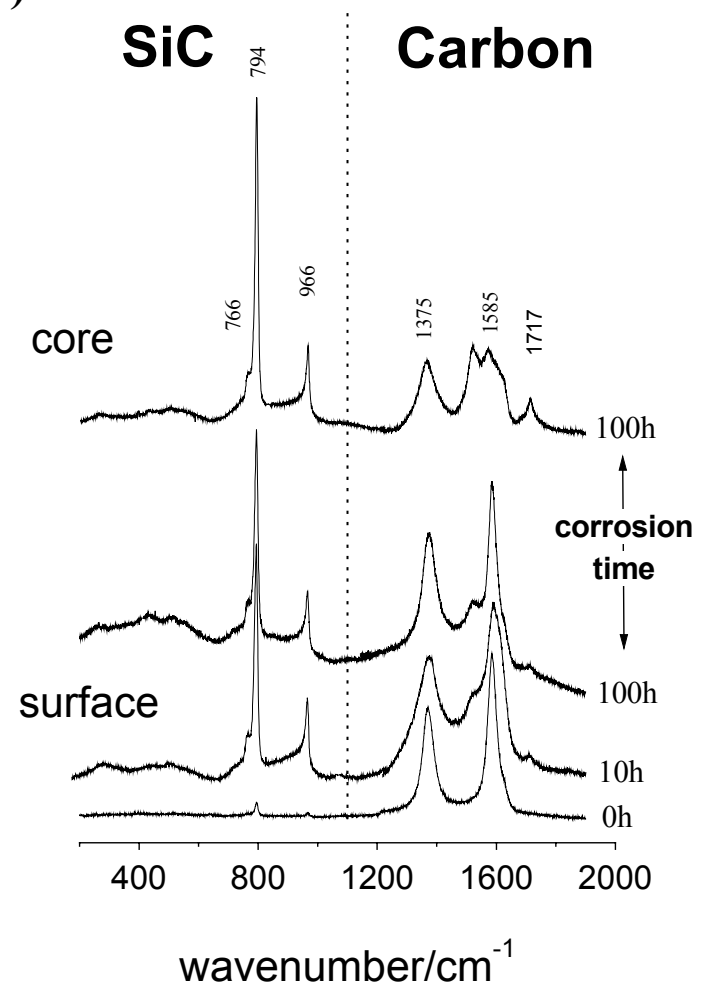

b)
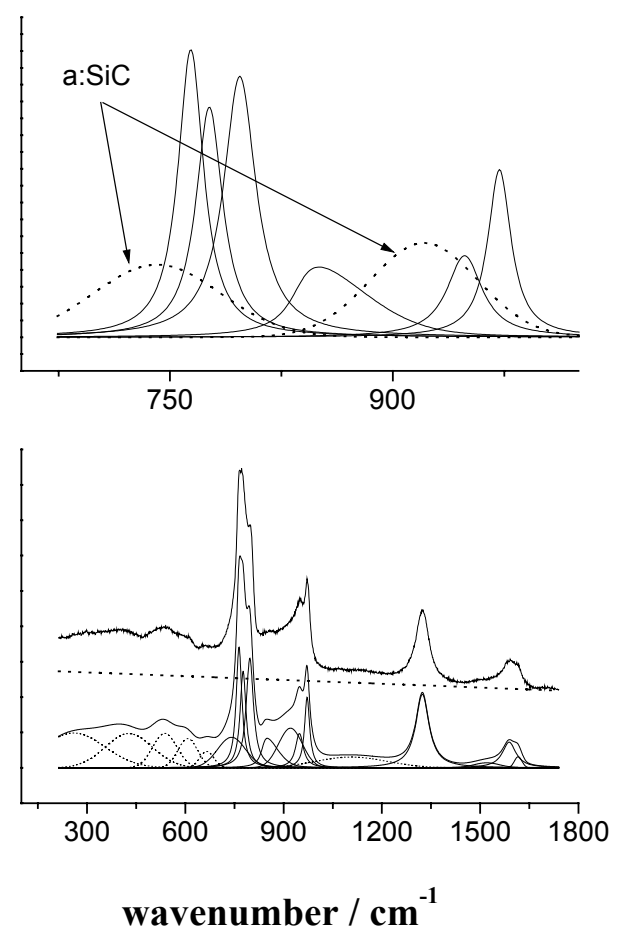

c)

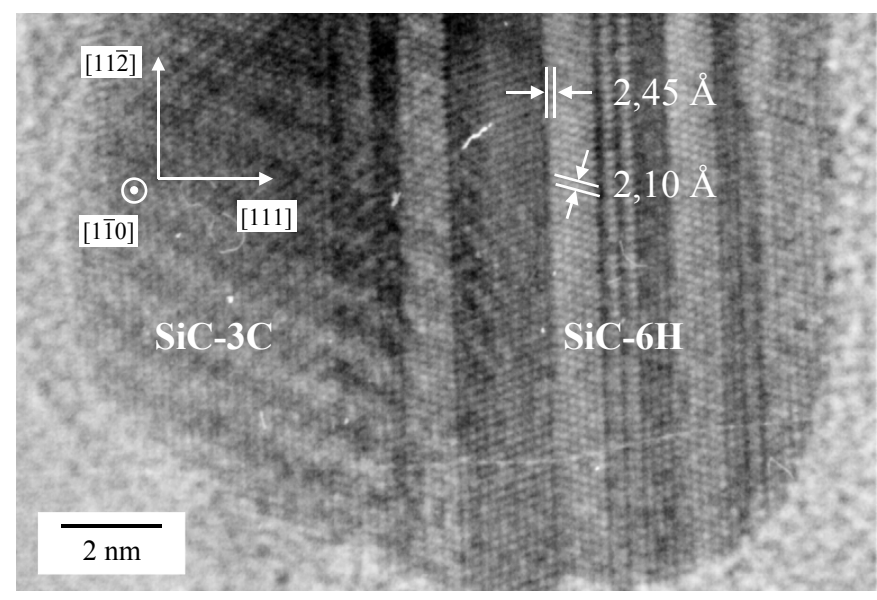

Figure 16 : a) Effect of alkaline corrosion by $\mathrm{NaNO}_{3}$ salt on the Raman spectrum of annealed NLM SiC fibres $\left(\lambda_{\text {laser }}=457.94 \mathrm{~nm}\right)$. Note the core spectrum was recorded on a fibre crosssection which has been exposed to corrosion (Adapted from Ph. Colomban et al., Materials and Corrosion, 53[5], pp 306-315, 2002) - b) Raman spectra recorded with $632.1 \mathrm{~nm}$ excitation on one NLM SiC fibre annealed for $10 \mathrm{hr}$, at $1600^{\circ} \mathrm{C}$, under reducing atmosphere.

The fit was obtained using the PCM [213,214] and a:SiC stands for "amorphous" silicon carbide - c) High Resolution Transmission Electron Microscopy (HRTEM) micrograph of one NLM fibre, after $10 \mathrm{hr}$ reducing annealing at $1600^{\circ} \mathrm{C}$ [210]. The picture shows one $\mathrm{SiC}$ nanocrystal embedded in amorphous SiC (Courtesy of M. Havel \& L. Mazerolles).

SiC contributions from the spectra in Figure 16-b were fitted according to the PCM (see $\S 3.3 .1$ ). This returned "coherence lengths" of $6.5 \pm 1.2 \mathrm{~nm}$ (TO) or $3.8 \pm 0.9 \mathrm{~nm}$ (LO), in good agreement with the size of $\mathrm{SiC}$ polytype domains appearing in Figure 16-c. We performed 
similar fits on spectra of Hi-S, SA and Sylramic nearly stoichiometric $3^{\text {rd }}$ generation $\mathrm{SiC}$ fibres [379], obtained from grafted polycarbosilane [371,380-383]. Only the Hi-S bands could not be adjusted at $\overrightarrow{\mathrm{k}}_{0}=\overrightarrow{0}$, indicating a greater stacking disorder. The coherence length was higher for Sylramic and SA fibres, in agreement with a higher grain size (100-500 [381] and 200 [384] nm, respectively, against $20 \mathrm{~nm}$ in the Hi-S [380]).
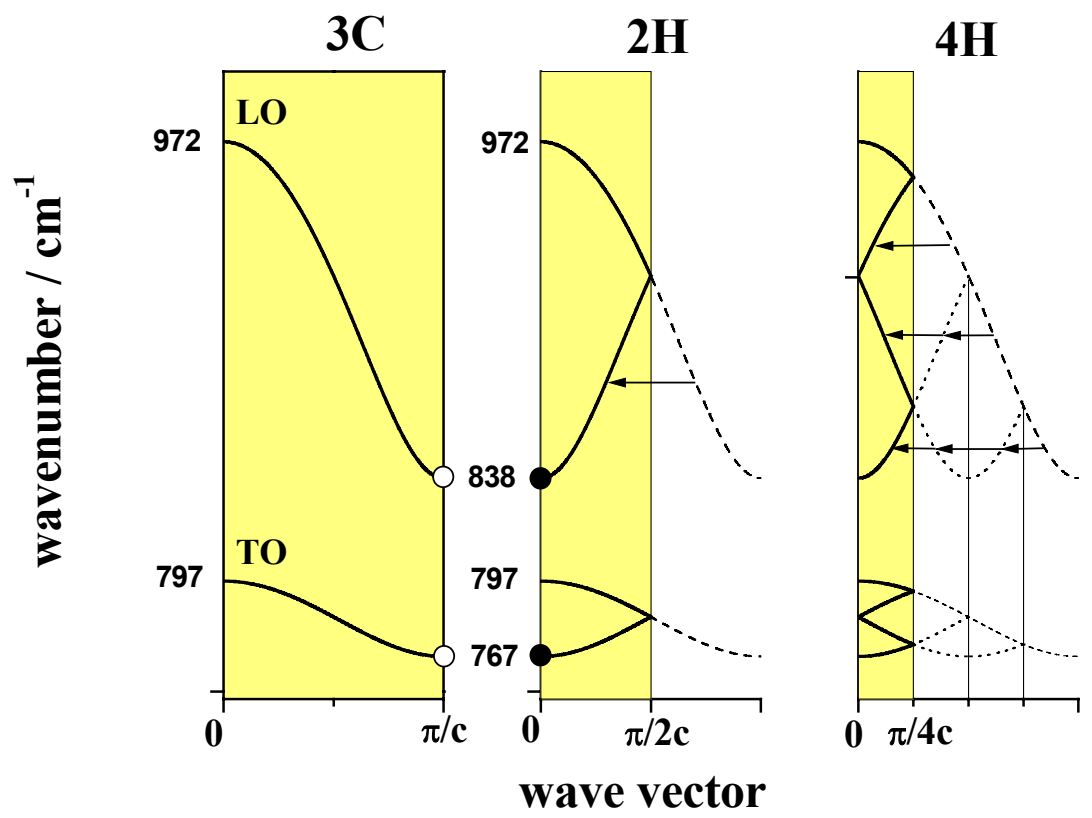

Figure 17 : Dispersion curves for the $3 \mathrm{C}, 2 \mathrm{H}$ and $4 \mathrm{H}$ polytypes, in equivalent directions of the reciprocal space. The "extended Brillouin zone" obtained by unfolding hexagonal (or rhombohedral) polytypes curves matches the "standard zone" of 3C SiC [245,385]. 3C-SiC modes marked with open circle are inactive but can be measured on the Raman spectra of $2 \mathrm{H}$ polytype (black circles) where they correspond to BZc.

\section{$\underline{4.5-\mathrm{CeO}_{2}}$}

The electrical properties and chemical reactivity of nano-crystalline $\mathrm{CeO}_{2}$ make it interesting for applications such as gas sensor, fuel cells electrolyte material, catalyst for oxygen storage, etc [154,197,216]. Ceria only first order Raman active mode is the triply degenerate symmetrical stretching vibration of the $\mathrm{CeO}_{8}$ vibrational unit at $\sim 465 \mathrm{~cm}^{-1}$. Only the oxygen atoms move in this mode [197,216,386], which is therefore very sensitive to the oxygen sublattice disorder resulting from processing and/or grain-size-induced non-stoichiometry. Spanier et al. simultaneously considered phonon confinement, stress, size distribution and non-stoichiometry as possible causes for grain size induced variations of ceria Raman spectra [216]. Figure 6 above showed the influence of grain size on the linewidth for ceria samples prepared on $\mathrm{Al}_{2} \mathrm{O}_{3}$ substrates, with a polymer precursor spin-coating process ensuring a remarkably homogeneous grain size distribution [387]. The broadening is obvious on Figure 18-a, which shows PCM-derived fits of Raman spectra [197]. The confinement lengths are plotted in Figure 18-b as a function of the grain size measured by X-ray diffraction: the extension of the homogeneous domains reaches a maximum for grain diameters around 100 $\mathrm{nm}$. Since a direct relationship is expected for the $465 \mathrm{~cm}^{-1}$ band with the oxygen sublattice, Kosacki et al. proposed that L corresponded to the average distance between oxygen 
vacancies. Below $100 \mathrm{~nm}$, the "modelled" correlation length decreases with the grain size following a cubic root law and this can be successfully accounted for by the reduction of vacancy formation enthalpy for nanocrystalline microstructures [197,387].

a)

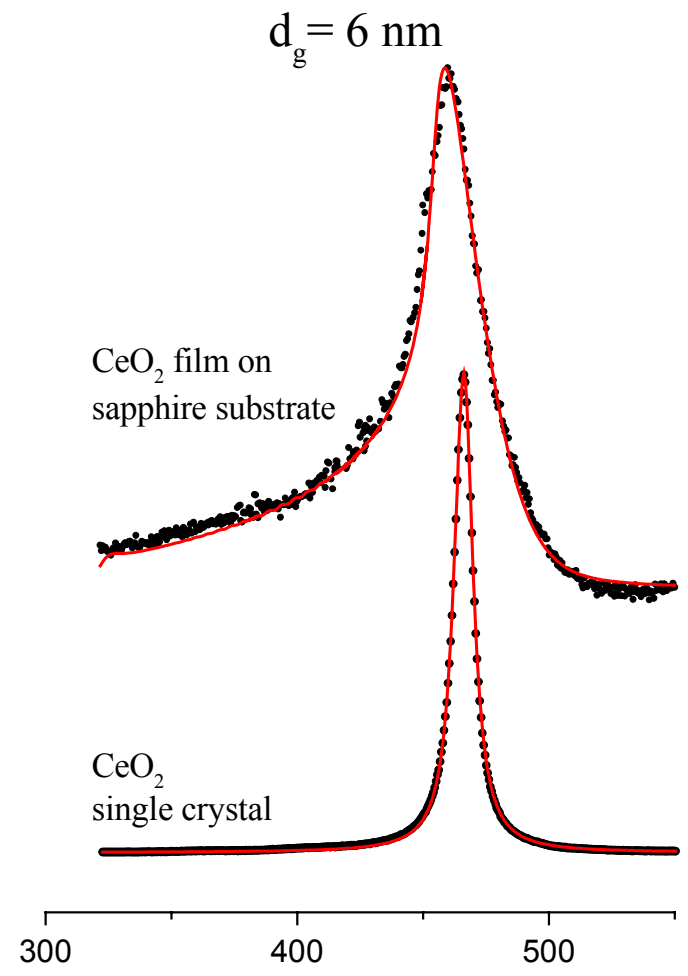

Wavenumber $/ \mathrm{cm}^{-1}$ b)

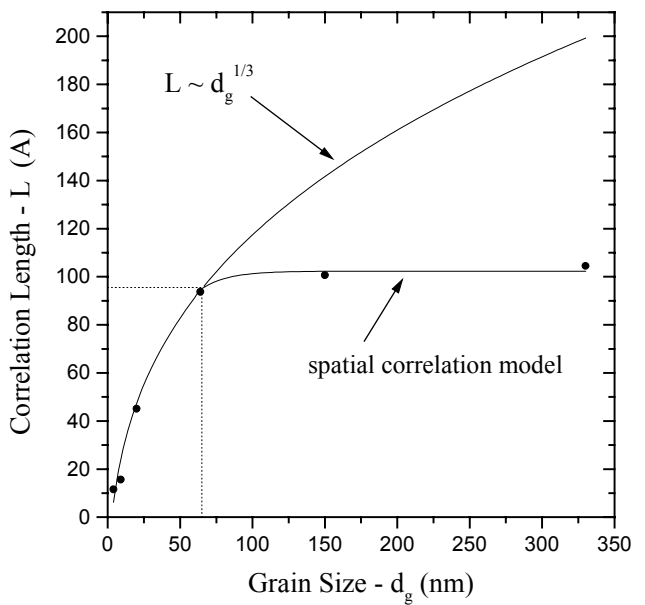

c)

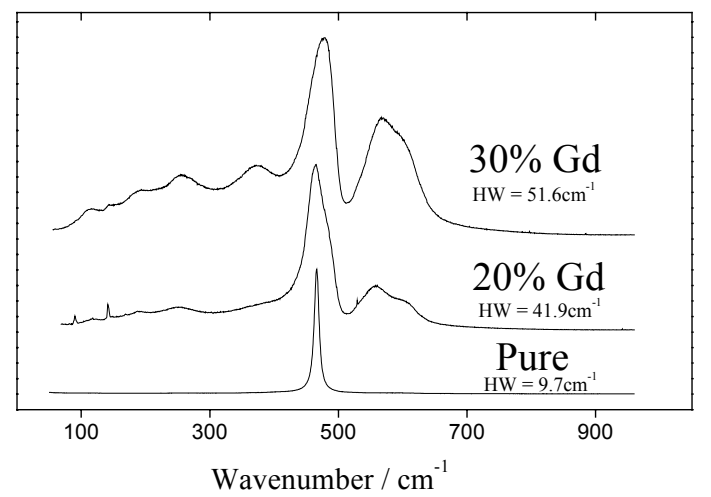

Figure 18 : a) Grain size effect on ceria Raman signature. The fits were obtained using the PCM (§ 3.3.1) - b) Comparison of the confinement length L obtained through the PCM with the grain size obtained by X-ray diffraction. Reprinted from Solid State Ionics, 149[1-2], I. Kosacki et al., Raman scattering and lattice defects in nanocrystalline $\mathrm{CeO}_{2}$ thin films, pp 99105, 2002, with permission from Elsevier - c) The spectra of gadolinium-doped ceria single crystals highly depend on the doping level (I. Kosacki and Ph. Colomban, Private Communication)

Figure 18-c shows the evidence for the stronger influence of substitutional disorder over the stoichiometric one. The broadening in $\mathrm{Gd}$-substituted $\mathrm{CeO}_{2}$ is indeed much more important than the one associated with grain size-induced non stoichiometry only (Figure 18-a). A separation of the short-range disorder and size-induced band broadening was similarly achieved for nano- $\mathrm{InVO}_{4}$ oxide (an electrode material for $\mathrm{Li}^{+}$intercalation) [26,27]. Note all samples in Figure 18-c are single crystals with the same highly symmetric structure. Their Xray diffractograms would look very much alike and only fine refinements would therefore take them apart. 


\section{THE MECHANICAL CHARACTERIZATION OF NANOPHASES BY MICRO-RAMAN SPECTROSCOPY}

A growing number of devices involve nanophased materials. Hence there is a need for in situ non destructive characterization methods. We have already seen that $\mu \mathrm{RS}$ nano-sensitivity to chemical bonds (stretching and bending modes) and their spatial arrangement (libration and external modes) makes it a good tool for probing crystallo-chemistry and reactions. We shall now demonstrate how nanoprecipitates can be used as "mechanical probes" [388-393]. In fact, very few experimental techniques lend themselves to micromechanical characterization of heterogeneous materials. Most of them are not specific and all of them have limitations. First, X-rays [394-396] and neutron [397,398] diffractions only work on crystallized samples. Ultrasound analysis gives a dynamical view of cracking rather than stress measurements [399-401], photoelastometry supposes perfect transparency [402,403] and SEM observations of resin grids only give an indirect measure of the surface deformations [404-406]. Other specific methods exist like instrumented (micro-nano)-indentation [407-411], fragmentation [412-417], "pull-out" [418], "push-out" [419] and "microdroplet" [414] tests but the validity of the models used for data interpretation is questionable due to their usually large number of parameters. Besides, these methods are sometimes inapplicable. For instance, carbon fibrereinforced composites can not be tested by conventional push-in micro-indentation because the fibres have a small diameter and cleave too easily.

\subsection{Raman Stress Sensitivity}

\section{$\underline{\text { 5.1.1. } \rightarrow \text { The Anharmonicity of Atomic Bonds }}$}

Below, we will assume that each stretching mode is specific to one given chemical bond. As long as elongation is limited, the bond can be modelled by a spring of length $l_{b}$, reduced mass $\mu$ and constant stiffness $\mathrm{k}_{\mathrm{b}}$. This is the so-called harmonic model where the interatomic potential has the form $\mathrm{V}\left(\mathrm{l}_{\mathrm{b}}\right)=\frac{\mathrm{k}_{\mathrm{b}}}{2}\left(1_{\mathrm{b}}-\mathrm{l}_{0}\right)^{2}$ :

$$
\begin{gathered}
\bar{v}_{\mathrm{vib}}=\frac{1}{2 \pi \mathrm{c}} \sqrt{\frac{\mathrm{k}_{\mathrm{b}}}{\mu}} \\
\mathrm{k}_{\mathrm{b}}=\frac{\partial^{2} \mathrm{~V}}{\partial \mathrm{l}_{\mathrm{b}}{ }^{2}}
\end{gathered}
$$

There is no $l_{b}$ dependence for the wavenumber in Eq. (37) and the harmonic potential does not predict any stress sensitivity for Raman bands. Yet such modelling rules out the phenomenon of bond dissociation $\left(l_{b} \rightarrow \infty\right)$ and does not correctly account for atoms non inter-penetrability $\left(l_{b} \rightarrow 0\right)$. In fact, the interaction potential should not be limited to a quadratic term and all real bonds are somewhat anharmonic (Figure 19). They include attractive and repulsive contributions, for which Mie and Grüneisen proposed the following expression, with $\mathrm{A}, \mathrm{R}, \mathrm{a}$ and $\mathrm{r}$ being positive constants [420]:

$$
\mathrm{V}\left(\mathrm{l}_{\mathrm{b}}\right)=\mathrm{V}_{0}+\mathrm{V}_{\text {attractive }}\left(1_{\mathrm{b}}\right)+\mathrm{V}_{\text {repulsive }}\left(\mathrm{l}_{\mathrm{b}}\right)=\mathrm{V}_{0}-\mathrm{A} \times \mathrm{l}_{\mathrm{b}}^{-\mathrm{a}}+\mathrm{R} \times \mathrm{l}_{\mathrm{b}}^{-\mathrm{r}}
$$


Table 1: Parameters of Mie-Grüneisen potentials in solids [421].

\begin{tabular}{c|c|c}
\hline Bonding & a & r \\
\hline Van der Waals & 6 & 12 \\
ionic & 1 & 9 \\
covalent & \multicolumn{2}{|c}{$\mathrm{a}+\mathrm{r} \sim 3$} \\
\cline { 2 - 3 }
\end{tabular}

In spite of its very significant influence on the physical behaviour of materials (only it can explain thermal expansion or the finite value of thermal conductivity [422]), anharmonicity is often considered as a simple perturbation: in what is referred to as the "quasi-harmonic" approximation, one considers that Eq. (37) and (38) are applicable to real potentials. According to Figure 19, this means atoms are bonded by strings whose stiffness $\left(\partial^{2} \mathrm{~V} / \partial \mathrm{l}_{\mathrm{b}}{ }^{2}\right)$ increases under compression and decreases under tension.

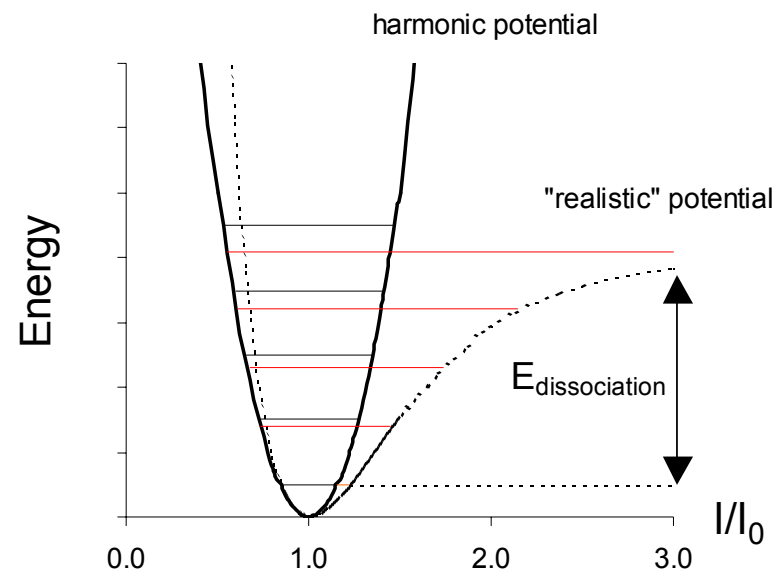

Figure 19 : Comparison of realistic and harmonic bond potentials.

In the case of the "quasi-harmonic" approximation, Eq. (39) is equivalent to:

$$
\frac{\mathrm{d} \bar{v}_{\text {vib }}}{\bar{v}_{0}}=\frac{\mathrm{dk}}{2 \mathrm{k}}=\frac{1}{2} \frac{\mathrm{a}(\mathrm{a}+1)(\mathrm{a}+2) \mathrm{A} \times \mathrm{l}_{\mathrm{b}}^{-(\mathrm{a}+3)}-\mathrm{r}(\mathrm{r}+1)(\mathrm{r}+2) \mathrm{R} \times \mathrm{l}_{\mathrm{b}}^{-(\mathrm{r}+3)}}{\mathrm{r}(\mathrm{r}+1) \mathrm{R} \times \mathrm{l}_{\mathrm{b}}^{-(\mathrm{r}+2)}-\mathrm{a}(\mathrm{a}+1) \mathrm{A} \times \mathrm{l}_{\mathrm{b}}^{-(\mathrm{a}+2)}} \mathrm{dl}_{\mathrm{b}}
$$

Introducing the bond elongation $\varepsilon_{1}=\left(1_{b}-1_{0}\right) / 1_{0}$, a Taylor expansion of $1_{b}{ }^{x}=1_{0}{ }^{x}\left(1+\varepsilon_{1}\right)^{\mathrm{x}}($ small strain assumption) and the requirement for the potential first derivative to be nil at $l_{b}=l_{0}$ yield:

$$
\begin{gathered}
\frac{d \bar{v}_{\text {vib }}}{\bar{v}_{0}}=-\frac{(\mathrm{a}+\mathrm{r}+3)}{2} \times \varepsilon_{\mathrm{l}_{\mathrm{b}}} \\
\bar{v}_{\text {vib }}=\bar{v}_{0}\left(1-\left(\frac{\mathrm{a}+\mathrm{r}+3}{2}\right) \times \varepsilon_{\mathrm{l}_{\mathrm{b}}}\right)
\end{gathered}
$$

There is therefore a direct proportionality factor between the wavenumber shift and bond deformation ${ }^{27}$ :

$$
\bar{v}_{\text {vib }}=\bar{v}_{0}+\mathrm{S}_{\mathrm{b}}^{\varepsilon} \times \varepsilon_{1_{\mathrm{b}}}^{\%}
$$

\footnotetext{
${ }^{27}$ The superscript "\%" will indicate deformation is given as a percentage.
} 
From a macroscopic point of view, a tensile (resp. compressive) stress is expected to decrease (resp. increase) $\bar{v}$. The effect will be more significant as the coefficients a and $r$ will be high, in other words as the bonds will be weak ${ }^{28}$.

\subsection{2. $\rightarrow$ Raman Spectroscopy of Single Crystals under Hydrostatic Compression}

Many RS experiments carried on stressed materials concern single crystals hydrostatically compressed in diamond anvil cells (DACs), where the pressure $\mathrm{P}_{\mathrm{h}}$ is transmitted by a fluid [424-426]. Wavenumbers then obey quasi-linear dependencies to $\mathrm{P}_{\mathrm{h}}[367,427-430]$ :

$$
\bar{v}_{\text {vib }}=\bar{v}_{0}+\mathrm{a} \times \mathrm{P}_{\mathrm{h}}+\mathrm{b} \times \mathrm{P}_{\mathrm{h}}^{2} \sim \bar{v}_{0}+\mathrm{a}^{\prime} \times \mathrm{P}_{\mathrm{h}}
$$

Such a behaviour indicates an equivalency between the macroscopic (Eq. (44)) and microscopic (Eq. (43)) responses to external solicitations. DAC experiments are usually used to measure the Grüneisen parameters of Raman modes [367,427-429,431-434]:

$$
\gamma_{\mathrm{m}}=-\frac{\partial \log \bar{v}_{\mathrm{m}}}{\partial \log \mathrm{V}}=\frac{1}{\beta_{\mathrm{T}}} \frac{\partial \log \bar{v}_{\mathrm{m}}}{\partial \mathrm{P}}
$$

In Eq. (45), $\mathrm{V}$ is the volume and $\beta_{\mathrm{T}}$ the isothermal compressibility. $\gamma_{\mathrm{m}}$ is almost independent of the mode in isotropic solids with one kind of bond controlling the lattice dynamics. This is the case for covalent/iono-covalent materials like diamond and 3D semiconductors from groups IV and III-V, where $\gamma$ is close to 1 [424,425]. It becomes about 2 in II-VI semiconductors and rises to 3 in alkali halides crystals [425]. In molecular crystals, $\gamma$ often changes from one mode to another [424] with, usually, $\gamma_{\text {internal modes }}<<\gamma_{\text {external modes. }}$

In an isotropic material under hydrostatic compression:

$$
\mathrm{P}_{\mathrm{h}}=\frac{\mathrm{dV}}{\mathrm{V}_{0}}=3 \varepsilon_{1}
$$

The combination of equations (41), (45) and (46) then leads to:

$$
\gamma=\frac{\mathrm{a}+\mathrm{r}+3}{6}
$$

The Grüneisen parameter then becomes a direct characteristic of interatomic potentials (a and $r$ parameters were introduced in Eq. (39)).

\subsection{3. $\rightarrow$ Raman Spectroscopy of Single Crystals under General Stress}

If a deformation is applied to a single crystal, vibrational mode independence is lost and phonon-phonon couplings occur [435]. The fundamental equation of Dynamics then becomes:

\footnotetext{
$\overline{28}$ The anharmonicity of the bonding potential is referred to as a "mechanical anharmonicity". Note that "electrical anharmonicity" also exists in RS. It corresponds to a second order Taylor expansion of the polarisability in Equation (2) [423].
} 


$$
\begin{gathered}
\mu \frac{\partial^{2} r_{i}}{\partial t^{2}}=-\sum_{j l m} \frac{\partial k_{i j}}{\partial \varepsilon_{l m}} \varepsilon_{l m} r_{j}=-\sum_{j l m} K_{i j l m} r_{j}=-\left[k_{i i} \times r_{i}+\sum_{\substack{j l m \\
j \neq i}} K_{i j l m} r_{j}\right] \\
\left\{\begin{array}{l}
i, j, l, m=x, y \text { or } z \\
r_{i}=\text { component of normal coordinate } \mathrm{r} \text { in direction } \mathrm{i} \\
\mu=\text { reduced mass }
\end{array}\right.
\end{gathered}
$$

The wavenumber shifts can then be directly linked to any macroscopic stress/strain via the elastic compliance tensor ${ }^{29}$ and the $\mathrm{K}_{\mathrm{ijlm}}$ coefficients that describe the strain-induced deformation of the bonding potentials; in other words: anharmonicity [435]. Few of these coefficients are independent in high-symmetry crystals (see [436] in cubic crystals or $[437,438]$ for hexagonal corundum). The mathematical expressions for the stress dependency of optical modes in cubic crystals can be found for given crystallographic directions $[90,435]$ and planes [439-443] $]^{30}$. Ager et Drory reported less than $15 \%$ disagreement between the biaxial residual stress measured by RS in a diamond film (deposited on a titanium alloy) using linear combination of uniaxial stresses perpendicular to the growth direction, and the theoretical value expected from elasticity theory [441]. The same kinds of measurements are common for semiconductors [444,445].

\section{$\underline{5.1 .4 .} \rightarrow$ The Case of Non-crystalline Materials}

In polycrystalline or amorphous materials, the tensorial formalism is irrelevant (stress directions are unknown). We must therefore adopt a "statistical" approach. Let $\theta_{\mathrm{b}}$ be the initial angle between a bond and the solicitation axis in a material macroscopically strained to $\Delta \varepsilon_{\mathrm{M}}{ }^{\%}$. The simplest assumption is to consider that this bond is strained by an amount of $\Delta \varepsilon_{\mathrm{b}}{ }^{\%}=\Delta \varepsilon_{\mathrm{M}}{ }^{\%}$ $\cos \theta_{\mathrm{b}}$. The resultant shift is an integration of Eq. (43) over all bonds:

$$
\Delta \bar{v}_{\text {tot }} \approx \mathrm{S}_{\mathrm{b}}^{\varepsilon} \times \int_{\theta=0}^{\theta=\frac{\pi}{2}}\left[\mathrm{p}(\theta) \times \Delta \varepsilon_{\mathrm{M}}^{\%} \cos (\theta)\right] \mathrm{d} \theta
$$

In Eq. (49), $p(\theta)$ represents the probability for $\theta_{b}$ to be $\theta$. If the material is isotropic, then $p(\theta)$ is a constant and:

$$
\Delta \bar{v}_{\text {total }} \sim \mathrm{S}^{\varepsilon} \times \Delta \varepsilon_{\mathrm{M}}^{\%}
$$

Therefore, a proportionality factor $\mathrm{S}^{\varepsilon}$, expressed in units of $\mathrm{cm}^{-1} / \%$, links the wavenumber shift to the macroscopic strain in isotropic materials. Besides, if Hooke's law applies over the full strain exploration ${ }^{31}$, then:

$$
\Delta \bar{v}=\mathrm{S}^{\varepsilon} \times \frac{100 \times \Delta \sigma}{\mathrm{E}}=\mathrm{S}^{\sigma} \times \Delta \sigma
$$

\footnotetext{
${ }^{29}$ Compliance terms $\mathrm{S}_{\mathrm{ij}}$ are defined according of Voigt's notation by $\varepsilon_{\mathrm{i}}=\mathrm{S}_{\mathrm{ij}} \times \sigma_{\mathrm{j}}(\varepsilon=$ strain; $\sigma=$ stress; $\mathrm{i}=1$ to 6 correspond to directions $\mathrm{xx}, \mathrm{yy}, \mathrm{zz}, \mathrm{yz}, \mathrm{xz}$ and $\mathrm{xy}$ respectively, with $\varepsilon_{4}=2 \times \varepsilon_{\mathrm{yz}}, \varepsilon_{5}=2 \times \varepsilon_{\mathrm{xz}}$ and $\left.\varepsilon_{6}=2 \times \varepsilon_{\mathrm{xy}}\right)$.

${ }^{30}$ Some errors in formulas from Ager et al. [441] and Cheong et al. [442] were listed in Reference [379].

${ }^{31}$ Hooke's law $(\sigma=\mathrm{E} \varepsilon)$ supposes a proportionality factor (the so-called Young's modulus) exists between stress $(\sigma)$ and deformation $(\varepsilon)$.
} 
The applied stress $\Delta \sigma$ and Young's modulus E are expressed in GPa. From hereon, the $S^{\varepsilon / \sigma}$ symbol, let us name it the Raman Mechanical Coefficient (RMC) ${ }^{32}$, will be used whenever comments apply to both $\mathrm{S}^{\sigma}$ and $\mathrm{S}^{\varepsilon} . \mathrm{S}^{\varepsilon / \sigma}$ is usually negative: wavenumbers decrease under tension and conversely ${ }^{33}$.

The technique of micro-Raman Extensometry ( $\mu \mathrm{RE}$ ) consists in deriving a $\mathrm{S}^{\varepsilon}$ value from a reference sample subjected to controlled strain (Figure 20) for later in situ strain determination in a composite [80,388,391-393,414] (see $\S 5.2$ and 5.3). The peculiarity of nanophased materials is that stress relaxation is expected at grain interfaces/interphases. The numerical values of the RMC end up being lower than in a single crystal. They are even lower if nanocrystals from a secondary phase are used as the mechanical probe: the more dispersed a phase, the lower its contribution to stress transfer and the lower the corresponding $S^{\varepsilon / \sigma}$ (Figure 21. For instance, $\mathrm{S}^{\sigma}$ for O-Si-O bending mode $\left(\sim 450 \mathrm{~cm}^{-1}\right.$; see Figure 11$)$ is $-5 \mathrm{~cm}^{-1} / \mathrm{GPa}$ in amorphous silica fibres, where tetrahedra are continuously bridged by oxygen atoms, but is 5 10 times lower for isolated rings of only 3-4 tetrahedra [446].

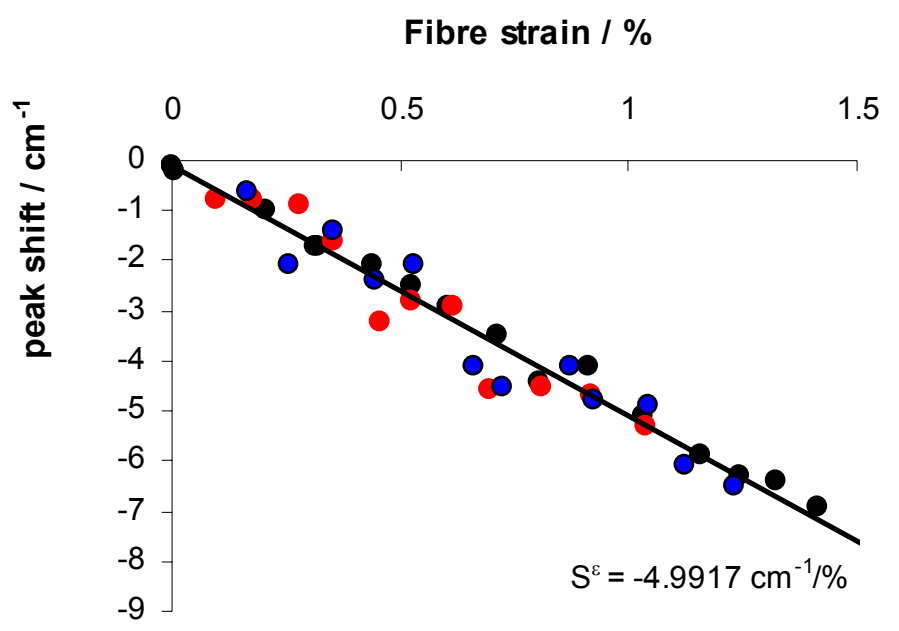

Figure 20 : Calibration of the Raman Mechanical Coefficient $\left(\mathrm{S}^{\varepsilon / \sigma}\right)$ of carbon $\mathrm{G}$ band (see $\S$ 4.3) for 3 IM7 fibres (Adapted from Ph. Colomban et al., Composites Part A, 37[4], pp 646651, 2006).

\subsection{In-situ micro-Raman Extensometry of Ceramic Fibre-reinforced Composites}

The reinforcement of ceramic materials with long ceramic fibres leads to low density and refractory materials of high damage tolerance. Therefore they should be an appropriate substitute for metal alloys in advanced engines (turbines) and waste treatment energy plants [366]. Ceramic fibres can also be directly incorporated in metal matrices to increase their high temperature mechanical properties [366]. These are often hard to measure and an optical nondestructive technique such as $\mu \mathrm{RE}$ will prove very useful in this regard. One of the peculiarities observed in ceramic fibres prepared from polymeric precursors is that there is no accumulation of impurities at the grain boundaries. Due to the lack of such usual diffusion

\footnotetext{
$\overline{32} \mathrm{~S}^{\varepsilon}$ is sometimes referred to as the Raman Frequency Gauge Factor (RFGF).

${ }^{33} \varepsilon$ and $\sigma$ are positive for tensile solicitations.
} 
regulators, abnormal grain growth is therefore very common and responsible for mechanical degradation [1]. A close relationship has been proven, for instance, between the microhardness and short-range ordering in sol-gel prepared nano-crystalline oxides [447] and some authors have already pointed out that the overall mechanical ability of fibres is governed by their microstructure [448-450]. The expertise of the fibre manufacturers consists in postponing the onset of crystallization and grain growth. The RMC difference between the reference single crystals and embedded nanoparticles under the same macroscopic stress can tell a lot about the fibre structure (Figure 21). Thus, $\mathrm{S}^{\varepsilon}$ is $-4.2 \mathrm{~cm}^{-1} / \%\left(\sim-1 \mathrm{~cm}^{-1} / \mathrm{GPa}\right.$ with $\mathrm{E}=$ $420 \mathrm{GPa}$ [370]) for SiC LO mode in the Hi-S fibre [379], which is lower than in SiC single crystals axially strained along [100] $\left(-1.5 \mathrm{~cm}^{-1} / \mathrm{GPa}\right)$ or [001] $\left(-3.0 \mathrm{~cm}^{-1} / \mathrm{GPa}\right)$ directions [379]. In such a quasi-stoichiometric $\mathrm{SiC}$ fibre, the carbon sensitivity is almost nil.

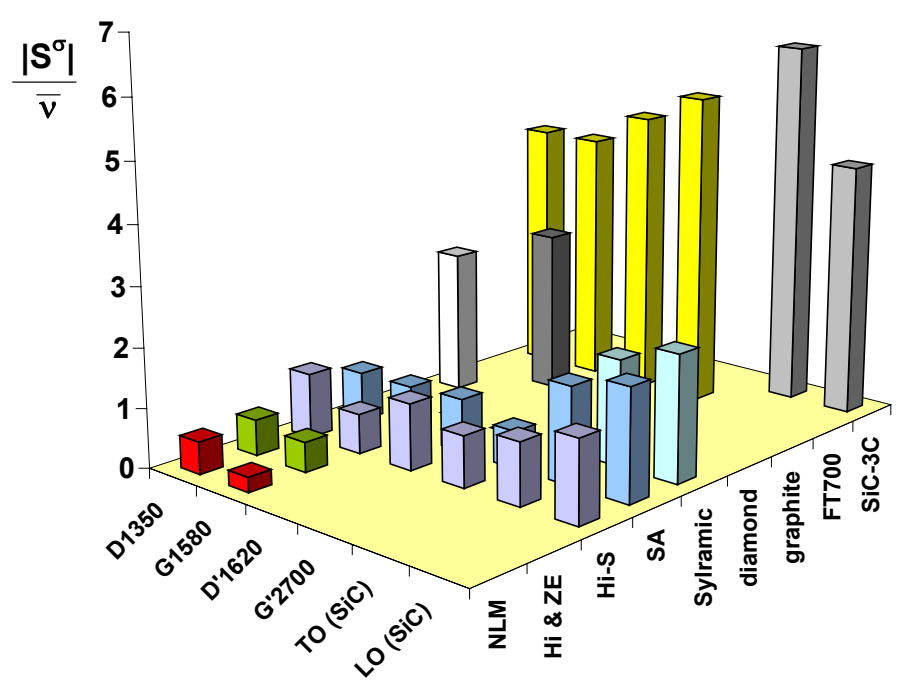

Figure 21 : Comparison of $S^{\sigma} / \bar{v}$ values for the $D, G, D^{\prime}$ and $G^{\prime}$ modes of carbon and the optical modes of SiC in different materials (NLM, Hi , Hi-S, SA, Sylramic: SiC fibers; FT700: carbon fiber). Reprinted by permission of Trans Tech Public Inc. from G. Gouadec and Ph. Colomban, Key Eng. Mater., 206-213, pp 617-620, 2002.

Equation (50), which derived from Eq. (43), assumed small bond deformation. Yet, the RMCs are measured up to the breaking point. For actual fibres, a better description of $S^{\varepsilon}$ is given with second or third order polynomial laws [451-453] and $S_{\text {Compression }}^{\varepsilon} S^{\varepsilon}$ Tension [389,453455]. Yet, the $S^{\varepsilon}$ Compression to $S^{\varepsilon}$ Tension ratio gets close to one in stiff materials (high Young's modulus) containing (iono)-covalent bonds and isotropic 3D-bonded inorganic structures. Such materials do indeed have a lower sensitivity to flaws than chain-like structures (where flaws open under tension and close under compression). Thus, the difference between $\mathrm{S}^{\varepsilon / \sigma}$ compression and $\mathrm{S}^{\varepsilon / \sigma}$ tension is lower in graphitic carbon fibres than in linear chain-based fibres (PPTA, PBZT). Pitch fibres have better aligned and closer graphene planes than PAN fibres. They also have more "reversible" $S^{\varepsilon}$ coefficients [392,417]. Micro-Raman Extensometry $(\mu \mathrm{RE})$ was most specifically applied to model polymer matrix systems reinforced with PAN [390,416,417,455-464] and Pitch [415,453] carbon fibres, aramid fibres [390,414,418,448,465-470], polyethylene fibres [471] or carbon nanotubes [472]. The matrix was then sufficiently transparent for in situ analysis of the embedded reinforcement material. 
The $S^{\varepsilon}$ calibrations and in-situ measurement of Raman spectra could be used to generate strain profiles $\varepsilon_{\mathrm{f}}(1)$ along the fibre, from which other properties like (longitudinal) residual strain $\left(\varepsilon_{\mathrm{f}}(1)\right.$ in the lack of external stress)[415,453,456,459,460,470,471], interfacial shear stress $\tau_{\mathrm{ci}}$ (Figure 22 and Ref. [459]), stress concentration factors [455,457-459,461,463,469]), Ineffective Lengths ${ }^{34}$ (IL) [459], the interfacial fracture energy $G_{i}$ [463] or the Coulombian friction coefficient (the proportionality factor between $\sigma_{\text {radial }}$ and $\tau_{\mathrm{ci}}$ ) [416] can be derived.

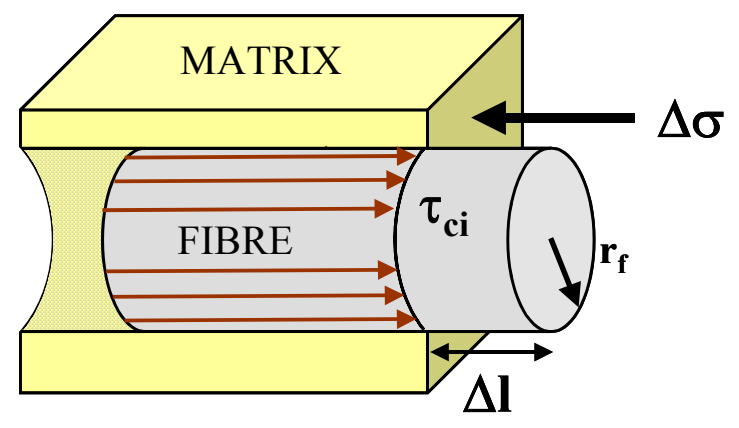

$$
\begin{aligned}
& \varepsilon_{\mathrm{f}}=\frac{\bar{v}_{\mathrm{vib}}-\bar{v}_{0}}{\mathrm{~S}^{\varepsilon}} \\
& \tau_{\mathrm{ci}}=-\mathrm{E}_{\mathrm{f}} \frac{\mathrm{r}_{\mathrm{f}}}{2}\left[\frac{\mathrm{d} \varepsilon_{\mathrm{f}}}{\mathrm{dl}}\right]
\end{aligned}
$$

Figure 22 : Diagram of Fibre-Matrix sliding. The deformations of the fibre and matrix when a stress $\Delta \sigma$ is applied to the composite are in the ratio of the Young's Moduli $\left(\Delta \sigma=\mathrm{E}_{\mathrm{f}} \times \Delta \varepsilon_{\mathrm{f}}=\right.$ $\left.\mathrm{E}_{\mathrm{m}} \times \Delta \varepsilon_{\mathrm{m}}\right)$. Hence the interfacial shear stress $\tau_{\mathrm{ci}}$ between the fibre and the matrix [459].

The key points for getting reliable $\mu \mathrm{RE}$ results are an accurate determination of $\mathrm{S}^{\varepsilon}$ (Figure 20) and taking into account that stress is not the only possible reason for peak shifts. Fibre/matrix inter-diffusion and laser-induced heating ${ }^{35}$ produce chemical and thermal shifts, which are accounted for in the following expression ${ }^{36}[91,379]$ :

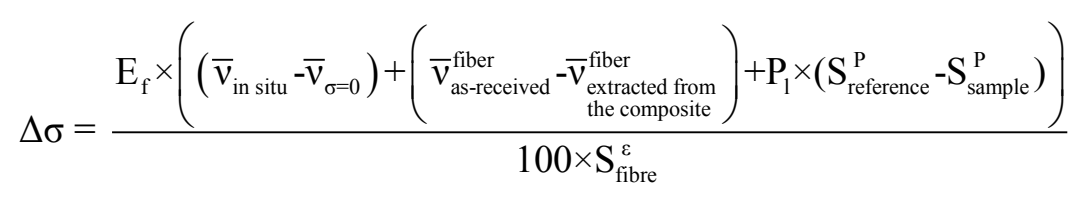

In Eq. (52), $S^{P}$ measures the (quasi-)linear dependence of the wavenumber on the laser power $\mathrm{P}_{1}$. The spatial resolution of $\mu \mathrm{RE}$ makes it easy to study the effect of a fibre environment (neighbouring fibres or cracks, fibre sizing, humidity, etc) on stress [455,457$460,466,469,470]$. The results fulfil relatively well the predictions of micromechanical models such as Cox's (axial stress for elastic fibre and matrix, the so-called "shear-lag" model) $[414,416]$, Piggott's (applies after fibre/matrix decohesion and for matrix creeping) $[414,418,466,474]$, the friction model of Kelly and Tyson $[416,460,474]$ or that of Cook and Gordon (stress concentration in the vicinity of cracks) [466].

The applicability of $\mu \mathrm{RE}$ to low transparency (ceramic matrix $[91,374,475]$ ) or intrinsically opaque (inter-metallic matrix [476]) coupons has recently been demonstrated, despite their high consolidation temperatures (fibre/matrix inter-diffusions might complicate the study). Given the much higher Young's modulus of such matrices with respect to resins, Ineffective Lengths (ILs) are small enough (a few $\mu$ m only instead of hundreds [459]) for a cross-section

\footnotetext{
$\overline{34}$ The length over which stress builds up from zero (at a fibre tip or a matrix crack) to its maximum value.

${ }^{35}$ Thermal expansion has the same effect on bond lengths (hence on $\bar{v}$ ) as a tensile stress [473].

${ }^{36}$ This equation neglects the statistical wavenumber dispersion in the reference and supposes wavenumbers are corrected for any instability of the Raman spectrometer.
} 
analysis to give results close to those obtained with longitudinal probing $[91,390,393,475,476]$. Yang and Young studied $\varepsilon_{\mathrm{f}}(1)$ in model single fibre NLM/pyrex $[474,477,478]$ and NLM/SiC [479] composites. Bollet et al. measured the influence of microcracking in SiC/glass-ceramic composites [480]. Pezzotti also used $\mu \mathrm{RE}$ to study cracking in "monolithic" ceramics [481]. Chollon and Takahashi studied a $\mathrm{C} / \mathrm{C}$ composite [482]. Colomban and co-workers measured residual stress in $\mathrm{C}_{\mathrm{f}} /$ mullite [475], NLM/mullite [475] and Hi-Nicalon/mullite [374,483] composites.

\subsection{In-situ Analysis of a $\mathrm{C}_{f}$ /epoxy Composite}

Nine $\mu \mathrm{m}$ diameter PAN-based low crystallinity IM7 carbon fibres (Hexcel Fibres, USA) were used for the unidirectional reinforcement of an epoxy matrix processed by radicalpolymerisation under $\gamma$ irradiation. The resulting composite $(\gamma$-pol) was nearly opaque, rather unfavourable conditions for a $\mu \mathrm{RE}$ study. These notwithstanding, $\gamma$-pol was submitted to a constant tensile strain of $1 \%$ and the spectra could be recorded through a few tens of micrometers of the matrix material, starting from the tip of an isolated fibre chosen in a fibrepoor region (Figure 23-a). This $\sim 300 \mu \mathrm{m}$-long segment was longer than the usual IL in polymer matrices $(\sim 200 \mu \mathrm{m}[391,457,459])$, which was mandatory to observe full fibrematrix interaction. The results of the spectra fitting are illustrated in Figure 23-b. D and G bands (see $\S 4.3$ ) have very similar wavenumber variations, with a few singular points. These might result from the local environment (presence of cracks or neighbouring fibres), as expected from the conclusions of van den Heuvel et. al. [457]. The jumps in opposite directions for $\mathrm{D}$ and $\mathrm{G}$ wavenumbers support an attribution to stresses of different nature (axial vs. radial). It is indeed a well established fact that graphite planes preferentially orientate along the longitudinal direction of high-performance carbon fibres [484,485]. $G$ wavenumber should thus be representative of the axial loading of the fibre. As for D band, it corresponds to defective graphene, possibly the $\mathrm{C}_{\mathrm{sp}}{ }^{2}-\mathrm{C}_{\mathrm{sp}}{ }^{3}$ bonds forming where graphite nanocrystals transform into amorphous carbon. Such bonds located at the surface and inbetween the graphite planes would be expected to be less specifically sensitive to the loading direction. $G$ wavenumbers have been converted into an axial compressive stress $\left(\sigma_{f}\right)$ in Figure 23-c and the resulting data were fitted according to the following model [366]:

$$
\sigma_{\mathrm{f}}=-\sigma_{\max } \frac{(1-\cosh (\beta(1 / 2-\mathrm{x})))}{\cosh \left(\frac{\beta 1}{2}\right)}-3.7
$$

$$
\beta=\frac{1}{r} \sqrt{\frac{E_{m}}{\left(1+v_{m}\right) E_{f} \ln \left(\frac{r_{m}}{r}\right)}}
$$

$\mathrm{x}$ is the position along the fibre of length 1 and radius $\mathrm{r}, \sigma_{\max }$ is the maximum stress, $v_{\mathrm{m}}$ is the matrix Poisson's ratio and $E_{f}$ and $E_{m}$ are the fibre and matrix Young's moduli. As for $r_{m}$, it corresponds to the effective matrix thickness responsible for the fibre stress. A value $\beta \sim 0.03$ $\mu \mathrm{m}^{-1}$ gave the best fit and the variation in Figure 23-c shows the axial compression rises from the tips along a characteristic IL $\sim 100 \mu \mathrm{m}$. 
a)
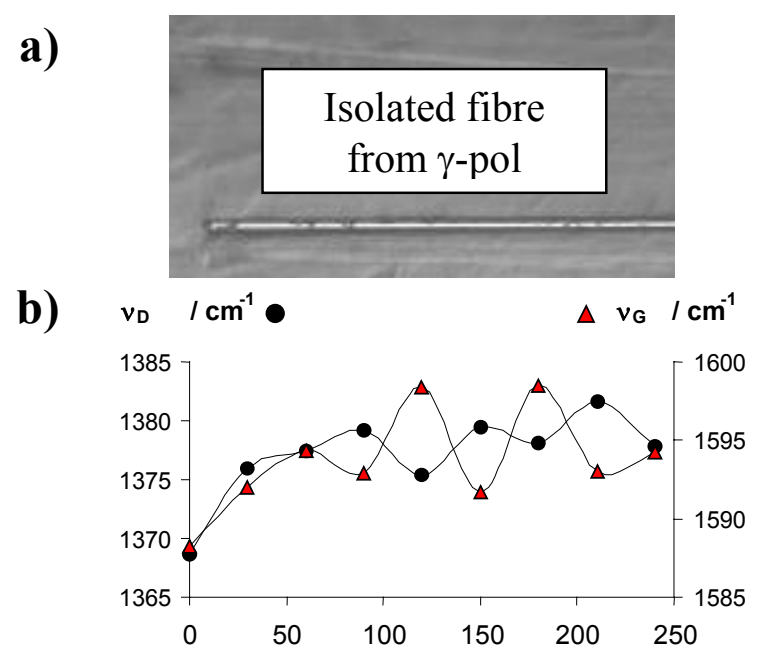

c)

Distance along the fibre / $\mu \mathrm{m}$

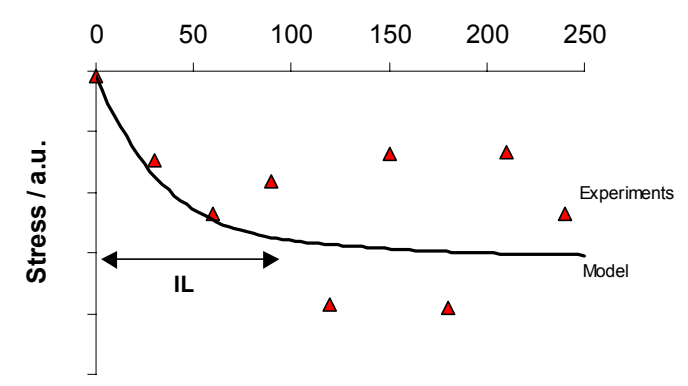

Figure 23 -Raman stress measurement in opaque industrial $\mathrm{C}_{\mathrm{f}} /$ epoxy composites submitted to tensile strain, Reprinted from Composites Part A, 37[4], Ph. Colomban et al., pp 646-651, 2006, with permission from Elsevier - a) Optical micrograph of an IM7 fibre entering the matrix of " $\gamma$-pol" composite (see text) strained to $1 \%$ - b) Wavenumber variations along the same fibre (origin at fibre tip, $\lambda_{\text {laser }}=457.9 \mathrm{~nm}, \mathrm{P}_{\text {illumination }}=1.5 \mathrm{~mW}$ ) - c) modelling of $v_{\mathrm{G}^{-}}$ derived axial compressive stress along the same fibre (IL: Ineffective Length) [486].

\section{4. "Micro-Macro" Correlations}

In this section, we will demonstrate how the local information accessed by RS through very simple spectra fittings correlates with "macroscopic" parameters such as Young's modulus, tensile strength and micro-hardness. The reason why such a correlation exists is the common dependence of the Raman signal and the mechanical behaviour on the micro/nanostructure of materials.

\subsection{1. $\rightarrow$ Comparison of Raman Spectra with Micro-Indentation Results}

Strong correlations between Raman and microhardness data have been reported by Gogotsi et al. for semiconductors, quartz and carbon [134,487] and by Amer et al. for "diamond-like carbon" [488]. Figure 24 illustrates how "mechanical changes" (Figure 24-c) are similarly related to a change in the Raman spectrum of either carbon or SiC (Figure 24-a\&b) in a SCS- 
6 fibre $^{37}$. The variations in Berkovich's micro-hardness $(\mu \mathrm{H}$; three-sided diamond tip) and Young's modulus were measured by Mann and co-workers [490] and we highlighted different zones with vertical dots. From core to periphery, they correspond to:

I)- The $32 \mu \mathrm{m}$-diameter carbon core consisting of graphitic units 1 to $5 \mathrm{~nm}$ in size [476].

II)- A $1.5 \mu \mathrm{m}$-thick layer of pyrolytic carbon (grains are $25-50 \mathrm{~nm}$ in size). It has been identified as the weakest part of the fibre because its graphitic planes are parallel to the fibre axis [490].

III)- A zone in which carbon coexists with $\mathrm{SiC}$ and evolves over $30 \mu \mathrm{m}$ towards a highly disordered carbon.

IV)- A zone where only $\mathrm{SiC}$ is detected (C-C bonds abundance $\leq 0.1 \%$ ). The $\mathrm{TO}$ and $\mathrm{LO}$ peak widening and the increase of the background level reveal stacking faults are all the more frequent as thickness increases [476]. In spite of this, the mechanical properties are constant, which suggests they are governed by free carbon in insertion.

V)- The interfacial carbon deposit (thickness $3 \mu \mathrm{m}$ ).

a)

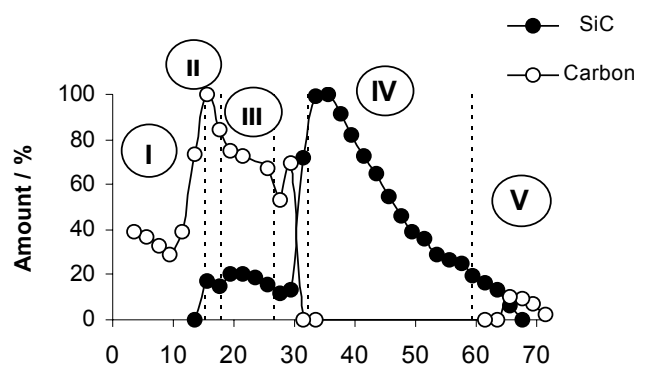

b)

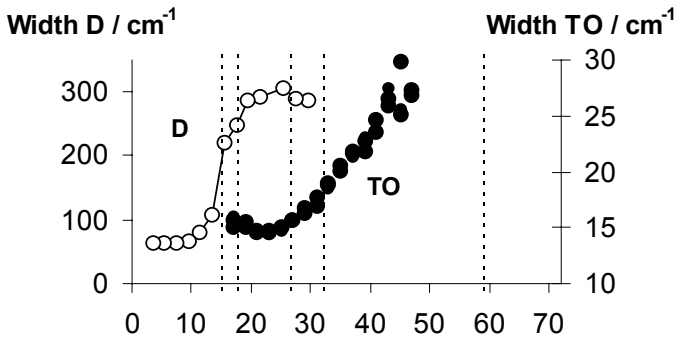

c)

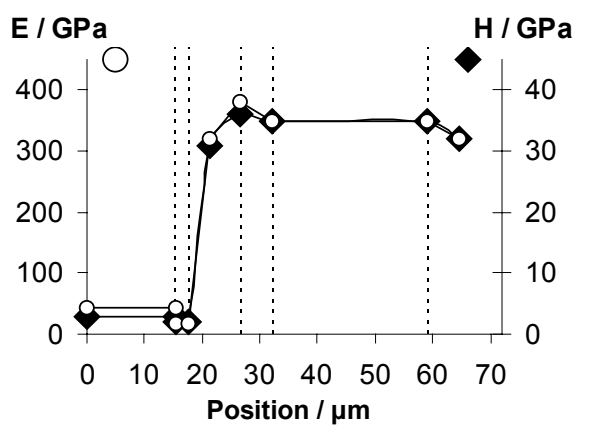

Figure 24 : Reprinted from J. Eur. Ceram. Soc., 21[9], G. Gouadec and Ph. Colomban, Nondestructive mechanical characterization of SiC fibres by Raman spectroscopy, pp 1249-1259, 2001, with permission from Elsevier - a) Raman detection of carbon (intensities of D, G and $\mathrm{D}^{\prime}$ peaks are added) and $\mathrm{SiC}$ (intensities of $\mathrm{TO}$ and $\mathrm{LO}$ peaks are added) as a function of the position along the fibre radius $\left(\lambda_{\text {laser }}=632.8 \mathrm{~nm}\right) .100 \%$ corresponds to the maximum intensity

\footnotetext{
${ }^{37}$ This is a $140 \mu \mathrm{m}$-diameter fibre used for metal-matrix reinforcement [366]. It has a high heterogeneity along the radius, due to a preparation by CVD of $\mathrm{SiC}$ (and carbon), on a carbon core [489].
} 
detected for each phase - b) Bandwidths obtained after spectra fitting for D carbon peak $\left(\lambda_{\text {laser }}=632.8 \mathrm{~nm}\right)$ and SiC TO modes (three separate scans; $\left.\lambda=514.5 \mathrm{~nm}\right)$ - c) Young's modulus and "Berkovich's hardness" (data from Mann et al [490]).

\subsection{2. $\rightarrow$ Comparison of Raman Spectra with Fibre Strength}

Figure 25 shows the correlation of the strength measured on NLM [371] and Hi-Nicalon [384] $\mathrm{SiC}$ fibres annealed at different temperatures (up to about $1500^{\circ} \mathrm{C}$ ) with their Raman spectra recorded at room-temperature. The linearity suggests that the macroscopic (strength measurement) and microscopic (Raman spectrum) responses to stress have the same dependence on grain size. A strength reduction resulting from grain boundary creeping is ruled out since it would not alter the Raman spectra. Two atmospheres were tested for the NLM grade. The mechanical failure is postponed in a reducing atmosphere but this does not seem to modify the "micro-macro" mechanical correlation.

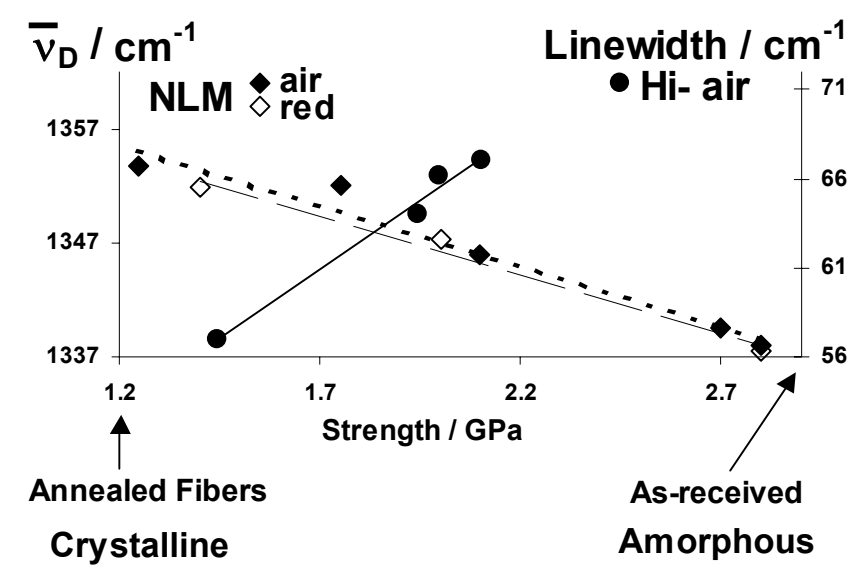

Figure 25 : Raman shift and linewidth of carbon D mode $(\lambda=514.5 \mathrm{~nm})$ plotted as a function of tensile strengths found in Berger et al. [384] or Kumagawa et al. [371] for heat treated SiC

fibres. Reprinted from J. Eur. Ceram. Soc., 21[9], G. Gouadec and Ph. Colomban, Nondestructive mechanical characterization of SiC fibres by Raman spectroscopy, pp 1249-1259, 2001, with permission from Elsevier.

\section{$\underline{\text { 5.3.3. } \rightarrow \text { Young Modulus and "Raman microextensometry" }}$}

At the macroscopic scale, fibres present the same tendency as their bonds to strengthen in compression and soften in tension [454]. Hooke's law being nothing but the macroscopic manifestation of bond stiffness, one may indeed expect to find a relationship linking $\mathrm{E}$ to $\mathrm{k}_{\mathrm{b}}$ (introduced in Eq. (37)) via $S^{\varepsilon}$.

The simplest expression modelling a "realistic" bond potential $\mathbf{V}\left(l_{b}\right)$ would be the following:

$$
\mathrm{V}\left(\mathrm{l}_{\mathrm{b}}\right)=\frac{\mathrm{k}_{\mathrm{b}}}{2}\left(1_{\mathrm{b}}-1_{0}\right)^{2}+\frac{\mathrm{k}_{\mathrm{b}}{ }^{\prime}}{6}\left(1_{\mathrm{b}}-1_{0}\right)^{3} ; \mathrm{k}^{\prime}<0
$$

With this form, the quasi-harmonic approximation (Eq. (37) and (38)) leads to:

$$
\bar{v}\left(1_{\mathrm{b}}\right) \propto \sqrt{\mathrm{k}_{\mathrm{b}} \times\left(1+\frac{\mathrm{k}_{\mathrm{b}}{ }^{\prime}}{\mathrm{k}_{\mathrm{b}}} \times\left(\mathrm{l}_{\mathrm{b}}-\mathrm{l}_{0}\right)\right)}
$$


$\mathrm{k}_{\mathrm{b}}$ ' is expected to be much smaller than $\mathrm{k}$ so, as long as $\mathrm{l}_{\mathrm{b}}$ remains close to $l_{0}$ (small strain assumption), a Taylor expansion is justified:

$$
\begin{gathered}
\bar{v}\left(1_{\mathrm{b}}\right) \sim \sqrt{\mathrm{k}_{\mathrm{b}}}+\frac{\mathrm{k}_{\mathrm{b}}{ }^{\prime}}{\sqrt{\mathrm{k}_{\mathrm{b}}}} \times\left(1_{\mathrm{b}}-\mathrm{l}_{0}\right) \\
\bar{v}\left(1_{\mathrm{b}_{2}}\right)-\bar{v}\left(1_{\mathrm{b}_{1}}\right) \sim \frac{\mathrm{k}_{\mathrm{b}}{ }^{\prime}}{\sqrt{\mathrm{k}_{\mathrm{b}}}} \times\left(1_{\mathrm{b}_{2}}-1_{\mathrm{b}_{1}}\right)
\end{gathered}
$$

Upon comparison with Eq. (50), this is equivalent to:

$$
\mathrm{S}_{\mathrm{b}}^{\varepsilon}=\frac{\mathrm{k}_{\mathrm{b}}{ }^{\prime} \mathrm{l}_{0}}{100 \sqrt{\mathrm{k}_{\mathrm{b}}}}<0
$$

Here again, we find that the energy shift for a strained bond can be expected to be proportional to the bond deformation. Besides, in such isotropic structures as most Nanophased fibres are, the "bond compression model" predicts a Young's modulus in the following form [491]:

$$
\mathrm{E}=\sum_{\text {bonds }} \frac{\mathrm{l}_{\mathrm{b}}^{2} \mathrm{k}_{\mathrm{b}}}{9}
$$

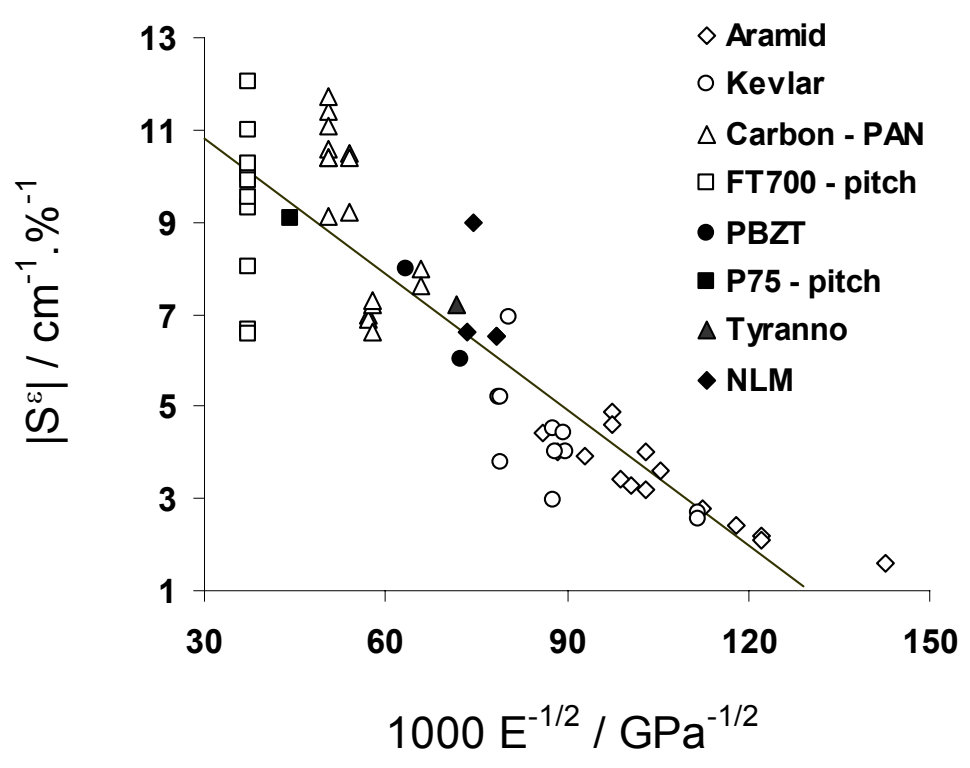

Figure 26 : Absolute value of the Raman Mechanical Coefficient (RMC) of carbon G band plotted versus the inverse of Young's modulus square root. Reprinted from J. Eur. Ceram.

Soc., 21[9], G. Gouadec and Ph. Colomban, Non-destructive mechanical characterization of SiC fibres by Raman spectroscopy, pp 1249-1259, 2001, with permission from Elsevier.

For fibers with a single (or dominant) type of bonds, $\mathrm{k}_{\mathrm{b}}$ and $\mathrm{k}_{\mathrm{b}}$ ' have unique values and, according to equations (58) and (59), $\mathrm{S}_{\mathrm{b}}{ }^{\varepsilon}$ must then be proportional to $\mathrm{E}^{-1 / 2}$. The same will apply to $S^{\varepsilon}$ (see discussion of Eq. (49) and (50)). Figure 26 plots $S_{G}{ }^{\varepsilon}$ coefficient measured in different carbon and "first generation" SiC fibres as a function of Young's modulus square root. The proportion and the distribution of the carbon and $\mathrm{SiC}$ phases differ in the fibres and, 
yet, a global classification is possible. Note that a direct proportionality between $\mathrm{S}^{\varepsilon}$ and $\mathrm{E}$ has been reported in the literature but these results were based on a narrower exploration of Young Modulus, limited to aramid [448,468,492], polyethylene [493] or carbon [454] fibres. Deviations from the linear trend in Figure 26 can be explained with the following arguments:

i)- A few of the $S^{\varepsilon}$ values appearing in Figure 26 were measured under compression and they are not necessarily comparable with those obtained through tensile straining. Indeed, $\mathrm{S}^{\varepsilon}$ coefficients suppose a linear dependence of Raman wavenumbers on the macroscopic deformation but a general relationship would rather be quadratic [451]:

$$
\Delta \bar{v}=\mathrm{a} \Delta \varepsilon+\mathrm{b}(\Delta \varepsilon)^{2}
$$

$\mathrm{S}^{\varepsilon}$ is thus subject to change when passing from tensile to compressive stress. There is almost no effect $(\mathrm{b} \sim 0)$ for isotropic structures like $\mathrm{SiC}$ fibres with strong covalent bonds in all directions but Melanitis et al. found a/b 5 (only !) in "PAN-based" carbon fibres [452].

ii)- Not all $\mathrm{S}^{\varepsilon}$ values in Figure 26 were obtained with the same laser line. Yet, carbon is a resonant species and both the spectrum and the penetration depth therefore depend on the wavelength. Most papers on Raman extensometry of carbon have neglected this aspect of the problem.

iii)- The reasoning that drove to Figure 26 neglected $\mathrm{k}_{\mathrm{b}}$ and $\mathrm{k}_{\mathrm{b}}$ ' distribution in the material. This simplification is probably acceptable in carbon fibres but certainly not in SiC fibres (Tyranno and NLM grades), where C-C and C-SiC bonds coexist. For that matter, $2^{\text {nd }}$ and $3^{\text {rd }}$ generation $\mathrm{SiC}$ fibres have such a specific behaviour that they were not even included in Figure 26. In such fibres with small amorphous or crystalline particles, C-C bonds are largely outnumbered by Si-C and inter-particles bonds. $\mathrm{E}_{\text {fibre }}$ (macroscopic scale) can no longer be considered as the counterpart of $\mathrm{S}^{\varepsilon / \sigma}$ particles (bond scale) [376].

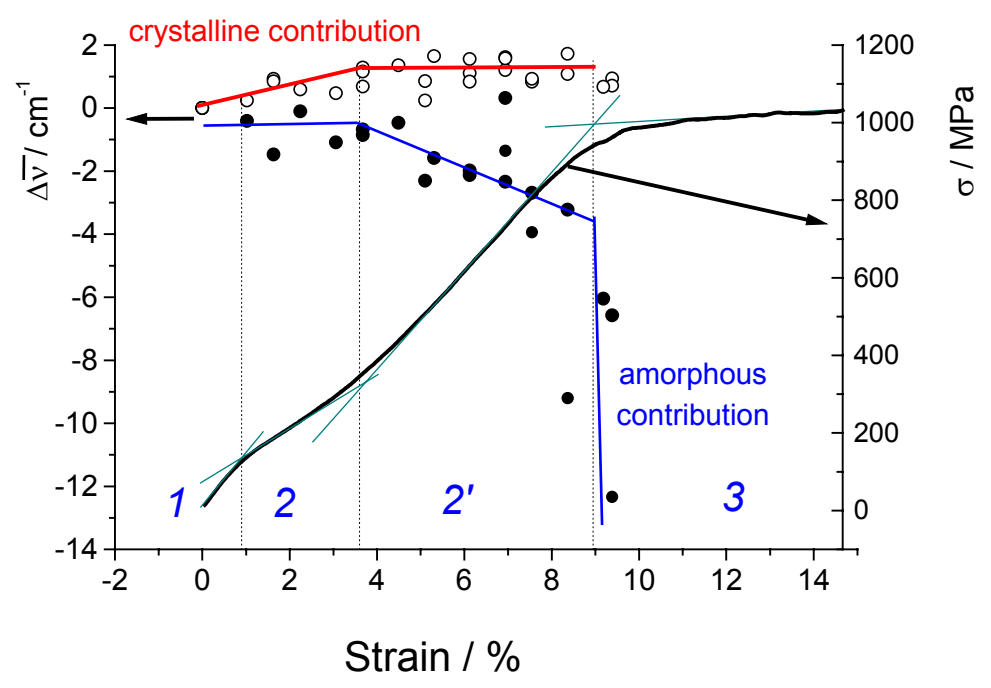

Figure 27 : Comparison of the strain dependency of the crystalline and amorphous low frequency Raman contributions (left hand scale) with the stress-strain tensile curve (right hand scale) for PET fibres. Reprinted from Engineering Fracture Mechanics, 73[16], Ph. Colomban et al., Micro-Raman study of the fatigue and fracture behaviour of single PA66 fibres: Comparison with single PET and PP fibres, pp 2463-2475, 2006, with permission from Elsevier. 
Figure 27 shows that the variations of Young Modulus (the slope on the strain-stress curve) in a semi-crystalline polymer such as PET can be correlated with the Raman shifts of the low frequency contributions corresponding to the amorphous and crystalline substructures (see Figure 5). In PET fibres, most of the stress is obviously transferred to the amorphous matrix, whereas nano-crystallites are only slightly compressed by a Poisson's effect in the amorphous matrix. In Polyamide fibres, both the amorphous and crystalline moieties accommodate the stress because the nanostructure puts them "in series". Wavenumber-strain curves then start with a plateau that corresponds to the disentanglement of the polymer chains (visco-elastic regime) and wavenumbers start downshifting as soon as "knots" block this process (elastic regime) $[170,171,173,494]$.

\subsection{CNTs used as Nanometric Stress Gauges}

On account of their very small dimension, the direct determination of CNTs Young's modulus is difficult. Most experimental values were obtained through manipulations with an AFM tip (see table 4 in Ref. [495]) but Lourie and Wagner demonstrated that an indirect measurement was possible using RS on "quenched" matrix-embedded nanotubes [496]. Using a "concentric cylinders" modelling of each CNT and the surrounding matrix, they calculated the axial tube stress due to cooling by $\Delta \mathrm{T}$ degrees and established nanotubes Young's modulus $\mathrm{E}_{\mathrm{NT}}$ :

$$
\mathrm{E}_{\mathrm{NT}}=\left[\frac{\Delta \alpha \Delta \mathrm{T}}{\varepsilon_{\mathrm{NT}}}-1\right] \frac{\left(1-\mathrm{f}_{\mathrm{NT}}\right)}{\mathrm{f}_{\mathrm{NT}}} \mathrm{E}_{\mathrm{m}}
$$

$\Delta \alpha$ is the difference in thermal expansion, $\mathrm{f}_{\mathrm{NT}}$ is the volume fraction of nanotubes, $\mathrm{E}_{\mathrm{m}}$ the Young's modulus of the matrix and $\varepsilon_{\mathrm{NT}}$ is the compressive strain. The latter was measured by the strain dependency of $\mathrm{D}^{*}$ Raman band of SWCNTs, which allowed Young's modulus determination. Conversely, some authors filled composites matrices with nanotubes and used them to sense fibre stress in polymers $[472,497,498]$. 


\begin{tabular}{|l|l|}
\hline Gwénaël Gouadec (born in 1973) is Associate Professor at Pierre \& \\
Marie Curie (Paris VI) University where he teaches Physical \\
Chemistry. He took an Engineering degree in Materials Science from \\
the University of Rennes in 1996 and served his French National Duty \\
period at ONERA, the French Aeronautics and Space Research Centre, \\
developing Sol-Gel interphases for $\mathrm{Al}_{2} \mathrm{O}_{3} / \mathrm{Al}_{2} \mathrm{O}_{3}$ Composites. In 2001, \\
he got a Ph.D on the "Physical and Chemical Characterization of \\
Aerospace Ceramic Matrix Composites by Raman (micro)- \\
Spectroscopy". In 2002, he was appointed post-doctoral fellow at the \\
University of Alabama at Birmingham. His Research project on the \\
nano-compressibility of carbon micro-ballons was funded by Los \\
Alamos National Laboratory (LANL). In 2003, Dr. Gouadec joined the \\
Laboratoire de Dynamique Interaction et Réactivité (LADIR). He now \\
specializes in the Raman spectroscopy of nanocrystalline and \\
amorphous domains found in fibres reinforcing Polymer and Ceramic \\
Matrix Composites (PMC/CMC).
\end{tabular}

\begin{tabular}{|l|l|}
\hline Philippe Colomban (born in 1952) is the Head of the "Laboratoire de \\
Dynamique, Interaction et Réactivité", a CNRS and Pierre \& Marie \\
Curie (Paris VI) University joint laboratory. He got is Engineering \\
degree from Sèvres "Ecole Nationale Supérieure de Céramique \\
Industrielle" in 1975 and a Ph. D. degree from Paris VI University in \\
1979. In 1975, Dr. Colomban was at Thomson-CSF (now Thalès) \\
Central Laboratory one of the first to develop Sol-Gel routes for \\
advanced ceramics (PLZT optically clear ferroelectric ceramics). In \\
1976 he was appointed as CNRS Research Associate at the "Ecole \\
Nationale Supérieure de Chimie de Paris" and studied superionic \\
conductors structure and ion mobility. In 1980 he created the Solid \\
State Chemistry Group at the "Ecole Polytechnique", focusing on \\
Nasicon solid electrolytes, proton conductors and Sol-Gel ceramics \\
and became a Consultant for the French Science and Technology \\
Minister. From 1989 to 1993 Dr. Colomban took charge of the new \\
projects at the Materials Department of ONERA, the French \\
Aeronautics and Space Research Establishment (Sol-Gel routes, \\
Oxide-oxide ceramic-matrix composites, microwave absorbing \\
materials and Functionally-Graded composites...). From 1994 to \\
present, as a CNRS Senior Scientist, his research specialized in the use \\
of Raman, IR and neutron spectroscopies for in situ stress and phase \\
distribution imaging (in composites, solid state devices, fuel cells, \\
synthetic and natural fibres) and the identification of the technologies \\
used for the fabrication of ancient ceramics and glasses. Philippe \\
Colomban was an invited speaker at International Conferences in \\
many different topics. His publications comprise more than 300 \\
original papers and reviews, and five patents. He is a board member of \\
Composite Part B Journal, Editor of Taoci (the series of the French \\
Oriental Ceramic Society) and received the Société Française de \\
Chimie (SFC) "Solid State Chemistry Award" in 1984.
\end{tabular}




\section{REFERENCES}

[1]Ph. Colomban, Gel Technology in Ceramics, Glass-Ceramics and Ceramic-Ceramic Composites, Ceramics International 15 (1989) 23.

[2]Ph. Colomban, G. Sagon and X. Faurel, Differentiation of Antique Ceramics from the Raman Spectra of their Colored Glazes and Paintings, J. Raman Spectrosc. 32 (2001) 351.

[3]J. Pérez-Arantegui, J. Molera, A. Larrea, T. Pradell, M. Vendrell-Saz, I. Borgia, B.G. Brunetti, F. Cariati, P. Fermo, M. Mellini, A. Sgamellotti and C. Viti, Luster Pottery from the Thirteenth Century to the Sixteenth Century: A Nanostructured Thin Metallic Film, J. Am. Ceram. Soc. 84 (2001) 442.

[4]Ph. Colomban and C. Truong, Non-destructive Raman Study of the Glazing Technique in Lustre Potteries and Faience (9-14th centuries): Silver ions, Nanoclusters, Microstructure and Processing, J. Raman Spectrosc. 35 (2004) 195.

[5]A.S. Edelstein and R.C. Cammarata (Eds.), Nanomaterials: Synthesis, Properties and Applications, CRC Press, Boca Raton, Fl, USA (1998).

[6]National Research Council, Small Wonders, Endless Frontiers, National Academy Press, Washington, DC, USA (2002).

[7]R.A. Andrievski and A.M. Glezer, Size Effects in Properties of Nanomaterials, Scripta Mater. 44 (2001) 1621.

[8]S.C. Tjong and H. Chen, Nanocrystalline Materials and Coatings, Mater. Sci. Eng. R45 (1-2) (2004) 1.

[9]A.A. Sirenko, J.R. Fox, I.A. Akimov, X.X. Xi, S. Ruvimov and Z. Liliental-Weber, In Situ Raman Scattering Studies of the Amorphous and Crystalline Si Nanoparticles, Sol. State Comm. 113 (2000) 553.

[10] M. Sternitzke, Structural Ceramic Nanocomposites, J. Eur. Ceram. Soc. 17 (1997) 1061.

[11] S. Gvasaliya, S. Lushnikov, B. Roessli and R. Katiyar, Raman and Neutron Scattering Study of $\mathrm{PbMg}_{1 / 3} \mathrm{Ta}_{2 / 3} \mathrm{O}_{3}$ Relaxor Ferroelectric, Ferroelectrics 302 (2004) 347.

[12] H.M. Jang, T.-Y. Kim and I.-W. Park, Nano-sized Clusters with Tetragonal Symmetry in $\mathrm{PbTiO}_{3}$-based Relaxor Ferroelectrics, Sol. State Comm. 127 (2003) 645.

[13] M.P. Thi, G. March and Ph. Colomban, Phase Diagram and Raman Imaging of Grain Growth Mechanisms in Highly Textured $\mathrm{Pb}\left(\mathrm{Mg}_{1 / 3} \mathrm{Nb}_{2 / 3}\right) \mathrm{O}_{3}-\mathrm{PbTiO}_{3}$ Piezoelectric Ceramics, J. Eur. Ceram. Soc. 25 (14) (2005) 3335.

[14] M. Iwata, N. Tomisato, H. Orihara, N. Arai, N. Tanaka, H. Ohwa, N. Yasuda and Y. Ishibashi, Raman Scattering in (1-x) $\mathrm{Pb}\left(\mathrm{Zn}_{1 / 3} \mathrm{Nb}_{2 / 3}\right) \mathrm{O}_{3-x} \mathrm{PbTiO}_{3}$ Mixed Crystal System II, Jap. J. Appl. Phys. 40 (2001) 5819.

[15] E. Stura and C. Nicolini, New Nanomaterials for Light Weight Lithium Batteries, Analytica Chimica Acta 568 (2006) 57.

[16] E. Stathatos, P. Lianos, V. Jovanovski and B. Orel, Dye-Sensitized Photoelectrochemical Solar Cells Based on Nanocomposite Organic-Inorganic Materials, J. Photochem. Photobio. 169 (2005) 57.

[17] National Research Council of the National Academies (AFSTB), Implications of Emerging Micro and Nanotechnology, The National Academies Press, Washington, DC, USA (2002).

[18] U. Simon and M.E. Franke, Electrical Properties of nanoscaled Host/Guest Compounds, Microporous and Mesoporous Mater. 41 (1-3) (2000) 1. 
[19] E. Rossinyol, J. Arbiol, F. Peiró, A. Cornet, J.R. Morante, B. Tian, T. Bo and D. Zhao, Nanostructured Metal Oxides Sinthesized by Hard Template Method for Gas Sensing Applications, Sensors and Actuators B109 (2005) 57.

[20] J.Y. Ying, A. Tschöpe and D. Levin, Synergistic Effects and Catalytic Properties Tailored by Nanostructure Processing, Nanostruct. Mater. 6 (1-4) (1995) 237.

[21] Y. Wang, Z. Tang and N.A. Kotov, Bioapplication of Nanosemiconductors, Materials Today 8 (2005) 20.

[22] G. Gouadec, Ph. Colomban and N.P. Bansal, Raman Study of Hi-Nicalon FiberReinforced Celsian Composites, Part1 : Distribution and Nanostructure of Different Phases, J. Am. Ceram. Soc. 84 (2001) 1129.

[23] Ph. Colomban, G. Gouadec and L. Mazerolles, Raman Analysis of Materials Corrosion : the Example of SiC Fibers, Materials and Corrosion 53 (2002) 306.

[24] J.-P. Schnell, G. Velasco, D. Dubreuil, D. Dieumegard, M. Croset and Ph. Colomban, Hydrogenated $\beta$-Alumina-Like Thin Film, Solid State Ionics 9/10 (1983) 1465.

[25] U. Lavrencic-Stangar, B. Orel, N. Groselj, Ph. Colomban, E. Statthatos and P. Lianos, In-situ Resonance Raman Studies of a Dye-Sensitized Photoelectrochemical Cell with a Sol-Gel Electrolyte, J. Electrochem. Soc. 149 (11) (2002) E413.

[26] A. Surca-Vuk, U. Opara-Krasovec, B. Orel and Ph. Colomban, Electrochromic and ExSitu IR and Raman Spectroelectrochemical Studies of $\mathrm{Li}_{x} \mathrm{InVO}_{4}$ Films Charged/Discharged in the $0<x<1$ Domain, J. Electrochem. Soc. 148 (2002) H49.

[27] A. Surca-Vuk, B. Orel, G. Drazic and Ph. Colomban, Vibrational Spectroscopy and Analytical Electron Microscopy Studies of Fe-V-O and In-V-O Thin Films (also in Chemical Monthly, 133 [6], 2002), In: Nanostruct. Mater. Eds. H. Hofman et al., Springer, Wien, 153 (2002).

[28] A. Cabot, A. Diéguez, A. Romano-Rodriguez, J.R. Morante and N. Barsan, Influence of the Catalytic Introduction Procedure on the Nano-SnO $\mathrm{O}_{2}$ Gas Sensor Performances. Where and How Stay the Catalytic Atoms?, Sensors and Actuators B79 (2001) 98.

[29] P. Cassoux, D. de-Caro, L. Valade, H. Casellas, S. Roques and J.-P. Legros, Thin Films and Nanowires of Molecule-Based Conductors and Magnets, Synthetic Materials 133-134 (2003) 659.

[30] G.J. Fang, K.-L. Yao and Z.-L. Liu, Fabrication and Electrochromic Properties of Double Layer $\mathrm{WO}_{3}(\mathrm{~V}) / \mathrm{V}_{2} \mathrm{O}_{5}($ Ti) Thin Films Prepared by Pulsed Laser Ablation Technique, Thin Solid Films 394 (2001) 64.

[31] V.V. Poborchii, Raman Spectra of Sulfur, Selenium or Tellurium Clusters Confined in Nano-Cavities of Zeolite A, Solid State Comm. 107 (1998) 513.

[32] Z. Wang, V. Pischedda, S.K. Saxena and P. Lazor, X-Ray Diffraction and Raman Spectroscopic Study of Nanocrystalline $\mathrm{CuO}$ under Pressure, Solid State Comm. 121 (2002) 275.

[33] J.C. Rodríguez-Cabello, L. Quintanilla and J.M. Pastor, Fourier Transform Raman Study of the Conformers in Poly(ethylene terephtalate), J. Raman Spectrosc. 25 (1994) 335.

[34] S. Bandow, Y. Maruyama, X.-X. Bi, R. Ochoa, J.M. Holden, W.-T. Lee and P.C. Eklund, Electronic and Vibrational Properties of Rb-intercalated $\mathrm{MoS}_{2}$ Nanoparticles, Mater. Sci. Eng. A204 (1995) 222.

[35] X. Li, W. Chen, C. Bian, J. He, N. Xu and G. Xue, Surface Modification of $\mathrm{TiO}_{2} \mathrm{Nano}^{-}$ Particles by Polyaniline, Appl. Surf. Sci. 217 (2003) 16.

[36] A.G. Kalampounias, D.T. Kastrissios and S.N. Yannopoulos, Structure and Vibrational Modes of Sulfur around the $\lambda$-Transition and the Glass-Transition, J. Non-Cryst. Sol. 326\&327 (2003) 115. 
[37] Ph. Colomban, Raman-Rayleigh Study of Nanophases, Materials Science Forum 453454 (2004) 269.

[38] G. Gouadec and Ph. Colomban, Non-Destructive Mechanical Characterization of SiC Fibers by Raman Spectroscopy, J. Eur. Ceram. Soc. 21 (2001) 1249.

[39] Ph. Colomban, Raman Analyses and "Smart Imaging" of Nanophases and Nanosized Materials, Spectrosc. Europe 15 (2003) 8.

[40] A.K. Arora, M. Rajalakshmi and T.R. Ravindran, Phonon Confinement in Nanostructures Materials, In: Encyclopedia of Nanoscience and Nanotechnology. Ed. H.S. Nalwa, American Scientific Publishers, 499 (2004).

[41] A. Singha, P. Dhar and A. Roy, A Nondestructive Tool for Nanomaterials: Raman and Photoluminescence Spectroscopy, Am. J. Phys. 73 (2005) 224.

[42] W.B. White, Structure of Particles and the Structure of Crystals: Information from Vibrational Spectroscopy., J. Ceram. Process. Res. 6 (2005) 1.

[43] Z.V. Popovic, Z. Dohcevic-Mitrovic, M. Stepanovic and M. Grujic-Brojcin, Nanopowders Characterization using the Vibrational Spectroscopy Methods, Proc. " $1{ }^{\text {st }}$ Inter. Workshop on Nanosci. \& Nanotechno." Belgrade, Serbia and Montenegro, 88 (2005).

[44] G. Gouadec and Ph. Colomban (Eds.), Special Issue on the Raman Study of Nanomaterials, J. Raman Spectrosc. 39 (2007).

[45] C. Kittel, Introduction to Solid State Physics, $7^{\text {th }}$ Edition, John Wiley and Sons, NY (1996).

[46] I.R. Lewis and H.G.M. Edwards (Eds.), Handbook of Raman Spectroscopy - From the Research Laboratory to the Process Line, Marcel Dekker Inc., NY, USA (2001).

[47] D.A. Long, Raman Spectroscopy, McGraw-Hill, New York (1977).

[48] R. Yakimova, T. Paskova, I. Ivanov, K. Germanova and M. Peev, Raman Scattering Study of Crystal Perfection of MOVPE-Grown GaAs, Semicond. Sci. Technol. 8 (1993) 179.

[49] H. Shen and F.H. Pollak, Raman Study of Polish-Induced Surface Strain in <100> GaAs and InP, Appl. Phys. Lett. 45 (1984) 692.

[50] J.-C. Lassegues, Incoherent Neutron Scattering Studies of Proton Conductors: from the Anhydrous Solid State to Aqueous Solutions, In: Proton Conductors. Solids, Membranes and Gels - Materials and Devices. Ed. Ph. Colomban, Cambridge University Press, Cambridge, U.K., 329 (1992).

[51] M. Bee, Localized and Long-Range Diffusion in Condensed Matter: State of the Art of QENS Studies and Future Prospects, Chem. Phys. 292 (2-3) (2003) 121.

[52] H. Poulet and J.P. Mathieu, Vibration Spectra and Symmetry of Crystals, Gordon and Breach, New York (1976).

[53] W. Hayes, G.F. Hopper and F.L. Pratt, Ionic Conductivity of Potassium $\beta$ " Alumina in the Very-Far Infrared, J. Phys. C: Solid State Phys. 15 (1982) L675.

[54] Ph. Colomban, Proton Conductors. Solids, Membranes and Gels - Materials and Devices, Cambridge University Press, Cambridge, U.K. (1992).

[55] M.J. Delaney and S. Ushioda, Comparison of the Raman Spectra of Superionic Conductors AgI and $\mathrm{RbAg}_{4} I_{5}$, Sol. State Comm. 19 (1976) 297.

[56] Ph. Colomban and G. Lucazeau, Vibrational Study and Conduction Mechanism in $\beta$ Alumina: (1) Stoichiometric $\beta$-Alumina, J. Chem. Phys. 72 (1980) 1213.

[57] Ph. Colomban, R. Mercier and G. Lucazeau, Vibrational Study of and Conduction Mechanism in $\beta$-Alumina: (2) Nonstoichiometric $\beta$-Alumina, J. Chem. Phys. 75 (1981) 1388. 
[58] K. Funke, R.D. Banhatti, S. Brückner, C. Cramer, C. Krieger, A. Mandanici, C. Martiny and I. Ross, Ionic Motion in Materials with Disordered Structures : Conductivity Spectra and the Concept of Mismatch and Relaxation, Phys. Chem. Chem. Phys. 4 (2002) 3155.

[59] K. Funke, High-Frequency Measurements and Interpretations, In: Solid Electrolytes. General Principles, Characterization, Materials, Applications. Eds. P. Hagenmuller and W. van Gool, Academic Press, New York, 77 (1978).

[60] X. Faurel, A. Vanderperre and Ph. Colomban, Pink Pigment Optimization by Resonance Raman Spectroscopy, J. Raman Spectrosc. 34 (2003) 290.

[61] Ph. Colomban, Lapis Lazuli as Unexpected Blue Pigment in Iranian Lâjvardina Ceramics, J. Raman Spectrosc. 34 (2003) 420.

[62] Ph. Colomban, Glasses, Glazes and Ceramics. Recognition of the Ancient Technology from the Raman Spectra, In: Raman Spectroscopy in Archaeology and Art History (Chap.13). Eds. H.G.M. Edwards and J.M. Chalmers, Royal Society of Chemistry, U.K., $192(2004)$.

[63] B.-K. Kim, J.-W. Hahn and K.R. Han, Quantitative Phase Analysis in Tetragonal-rich Tetragonal/Monoclinic two Phase Zirconia by Raman Spectroscopy, J. Mater. Sci. Lett. 16 (1997) 669.

[64] L.M. Fraas, S.P.S. Porto and E. Loh, Symmetry in Raman Scattering from the Optical Phonon in Single Crystal Beryllium, Sol. State Comm. 8 (1970) 803.

[65] M.V. Abrashev and M.N. Iliev, Polarized Raman Spectra of $\mathrm{Y}_{2} \mathrm{BaCuO}_{5}$ : Normal-mode Assignment from Substitutions for Y and Ba, Phys. Rev. B45 (14) (1992) 8046.

[66] C. Thomsen and M. Cardona, Raman Scattering in High- $T_{C}$ Superconductors, In: Physical Properties of High Temperature Superconductors I. Ed. D.M. Ginzberg, World Scientific, Singapore, 409 (1989).

[67] P. D'Arco and B. Piriou, Fluorescence Spectra of Eu ${ }^{3+}$ in Synthetic Polycrystalline Anorthite; Distribution of Eu ${ }^{3+}$ in the Structure, Amer. Miner. 74 (1989) 191.

[68] G. Gouadec, Ph. Colomban, N. Piquet, M.-F. Trichet and L. Mazerolles, Raman $/ \mathrm{Cr}^{3+}$ Fluorescence Mapping of a Melt-grown $\mathrm{Al}_{2} \mathrm{O}_{3} / \mathrm{GdAlO}_{3}$ Eutectic, J. Eur. Ceram. Soc. 25 (2005) 1447.

[69] G. Turrell and J. Corset (Eds.), Raman Microscopy. Developments and Applications, Academic Press, London (1996).

[70] A. Paipetis, C. Vlattas and C. Galiotis, Remote Laser Raman Microscopy (ReRaM). 1Design and Testing of a Confocal Microscope, J. Raman Spectrosc. 27 (1996) 519.

[71] Renishaw Spectrosc. Products Division, Drug Detection using Raman Spectroscopy Simple, Rapid Identification and Characterisation of Illicit Substances, AN/097 (2004).

[72] L. Rayleigh, Investigations in Optics with special reference to the Spectroscope, Phil. Mag. 8 (1879) 261.

[73] M. Born and E. Wolf, Principles of Optics, Pergamon Press, Oxford, 441 (1985).

[74] A.E. Conrady, Applied Optics and Optical Design, Part 2, Dover Publications, 627 (1960).

[75] N.T. Goldsmith, Deep Focus; a Digital Image Processing Technique to Produce Improved Focal Depth in Light Microscopy, Image Anal. Stereol. 19 (2000) 163.

[76] M. Havel and Ph. Colomban, Smart Raman and Rayleigh Spectroscopy for the Analysis of Nanomaterials, Microsc. Analysis 20 (2006) 13.

[77] C.H. Shek, G.M. Lin and J.K. Lai, Effect of Oxygen Deficiency on the Raman Spectra and Hyperfine Interactions of Nanometer $\mathrm{SnO}_{2}$, Nanostruct. Mater. 11 (1999) 831.

[78] H. Kuzmany and P. Knoll, Application of the Particle in the Box Model for Resonance Raman Scattering to Recent Experimental Results of Polyacetylene, Mol. Cryst. Liq. Cryst. 117 (1985) 385. 
[79] J. Kürti and H. Kuzmany, Resonance Raman Scattering from Finite and Infinite Polymer Chains, Phys. Rev. B44 (1991) 597.

[80] Ph. Colomban, Raman Microspectrometry and Imaging of Ceramic Fibers in CMCs and MMCs, Ceram. Trans. 103 (2000) 517.

[81] D. Routkevich, T.L. Haslett, L. Ryan, T. Bigioni, C. Douketis and M. Moskovits, Synthesis and Resonance Raman Spectroscopy of CdS Nano-Wire Arrays, Chem. Phys. 210 (1996) 343.

[82] C. Raptis, D. Nesheva, Y.C. Boulmetis, Z. Levi and Z. Aneva, Exciton Related Resonant Raman Scattering from CdSe Quantum Dots in an Amorphous GeS $S_{2}$ Thin Film Matrix, J. Phys.: Cond. Matter 16 (2004) 8221.

[83] S. Folch, A. Regis, A. Gruger and Ph. Colomban, Chain Length Effect on Intrachain Electronic Excitation and Interchain Coupling in Poly- and Oligo-anilines, Synth. Metals 110 (2000) 219.

[84] J. Godlewski, P. Bouvier, G. Lucazeau and L. Fayette, Stress Distribution Measured by Raman Spectroscopy in Zirconia Films Formed by Oxidation of Zr-Based Alloys, ASTM STP 1354 (2000) 877.

[85] G. Lucazeau, Quelques Exemples d'Application de la Spectroscopie Raman en Physique et en Chimie du Solide, Proc. "Ecole Thématique de Spectroscopie Raman en Chimie et Physique des Matériaux", Autrans, France, (1998).

[86] P. Bouvier, Mesures de Contraintes dans des Couches d'Oxydation d'Alliages de Zirconium, Proc. "Ecole Thématique de Spectroscopie Raman en Chimie et Physique des Matériaux", Autrans, France, (1998).

[87] A. El-Khalki, A. Gruger and Ph. Colomban, Bulk / Surface Nanostructure and Defects in Polyaniline Films and Fibres, Synth. Metals 139 (2003) 215.

[88] Ph. Colomban, S. Folch and A. Gruger, Vibrational Study of Short-Range Order and Structure of Polyaniline Bases and Salts, Macromolecules 32 (1999) 3080.

[89] G. Gouadec, S. Karlin and Ph. Colomban, Raman Extensometry Study of NLM202 and Hi-Nicalon SiC Fibres, Composites B29 (1998) 251.

[90] I. De Wolf, Stress Measurements in Si Microelectronics Devices using Raman Spectroscopy, J. Raman Spectrosc. 30 (10) (1999) 877.

[91] G. Gouadec, Ph. Colomban and N.P. Bansal, Raman Study of Hi-Nicalon FiberReinforced Celsian Composites, Part2 : Residual Stress in the Fibers, J. Am. Ceram. Soc. 84 (2001) 1136.

[92] Y.K. Kim and H.M. Jang, Polarization Leakage and Asymmetric Raman Line Broadening in Microwave Dielectric $\mathrm{ZrTiO}_{4}$, J. Phys. Chem. Solids 64 (2003) 1271.

[93] S. Loridant, G. Lucazeau and T. Le-Bihan, A High-Pressure Raman and X-Ray Diffraction Study of the Perovskite $\mathrm{SrCeO}_{3}$, J. Phys. Chem. Solids 63 (2002) 1983.

[94] M.N. Iliev, M.V. Abrashev, H.-G. Lee, V.N. Popov, Y.Y. Sun, C. Thomsen, R.L. Meng and C.W. Chu, Raman Spectroscopy of Orthorhombic Perovskitelike $\mathrm{YMnO}_{3}$ and $\mathrm{LaMnO}_{3}$, Phys. Rev. B 57 (1998) 2872.

[95] D.G. Georgiev, P. Boolchand and K.A. Jackson, Intrinsic Nanoscale Phase Separation of Bulk $\mathrm{As}_{2} \mathrm{~S}_{3}$ Glass, Phil. Mag. 83 (25) (2003) 2941.

[96] W.P. Griffith, Raman Studies on Rock-Forming Minerals. Part II. Minerals Containing $\mathrm{MO}_{3}, \mathrm{MO}_{4}$, and $\mathrm{MO}_{6}$ Groups, J. Chem. Soc. (A) (1970) 286.

[97] W.P. Griffith, Raman Spectroscopy of Minerals, Nature 224 (1969) 264.

[98] W.P. Griffith, Raman Studies on Rock-Forming Minerals. Part I. Orthosilicates and Cyclosilicates, J. Chem. Soc. (A) (1969) 1372. 
[99] P. Gillet, V. Sautter, J. Harris, B. Reynard, B. Harte and M. Kuntz, Raman Spectroscopic Study of Garnet Inclusions in Diamonds from the Mantle Transition Zone, Am. Miner. 87 (2002) 312.

[100] T. Chaplin, G.D. Price and N.L. Ross, Computer Simulation of the Infrared and Raman Activity of Pyrope Garnet, and Assignment of Calculated Modes to Specific Atomic Motions, Am. Miner. 83 (1998) 841.

[101] D.N. Argyriou, H.N. Bordallo, B.J. Campbell, A.K. Cheetham, D.E. Cox, J.S. Gardner, K. Hanif, A. dos Santos and G.F. Strouse, Charge Ordering and Phase Competition in the Layered Perovskite $\mathrm{LaSr}_{2} \mathrm{Mn}_{2} \mathrm{O}_{7}$, Phys. Rev. B (22) (2000) 15269.

[102] L.-G. Hwa, S.-L. Hwang and L.-C. Liu, Infrared and Raman Spectra of Calcium Alumino-Silicate Glasses, J. Non-Cryst. Sol. 238 (1998) 193.

[103] W.P. Griffith, Raman Spectroscopy of Terrestrial Minerals, In: Infrared and Raman Spectroscopy of Lunar and Terrestrial Minerals. Ed. J. C. Carr, Academic Press, New York, 299 (1975).

[104] K. Nakamoto, Infrared and Raman Spectra of Inorganic and Coordination Compounds, John Wiley and Sons, New York (1986).

[105] A. Otto, Surface-Enhanced Raman Scattering of Adsorbates, J. Raman Spectrosc. 22 (1991) 743.

[106] T.M. Cotton, J.-H. Kim and G.D. Chumanov, Application of Surface-Enhanced Raman Spectroscopy to Biological Systems, J. Raman Spectrosc. 22 (1991) 729.

[107] J. Zhang, X. Li, X. Sun and Y. Li, Surface Enhanced Raman Scattering Effects of Silver Colloids with Different Shapes, J. Phys. Chem. B109 (2005) 12544.

[108] J.-L. Yao, J. Tang, D.-Y. Wu, D.-M. Sun, K.-H. Xue, B. Ren, B.-W. Mao and Z.-Q. Tian, Surface Enhanced Raman Scattering From Transition Metal Nano-Wire Array and the Theoretical Consideration, Surface Sci. 514 (2002) 108.

[109] Z.-Q. Tian (Ed.), Special Issue on Surface Enhanced Raman Spectroscopy, J. Raman Spectrosc. 36 (6-7) (2005).

[110] M.P. Thi, Microprobe Study of Enhanced Raman Scattering Effect on $\mathrm{WO}_{3} / \mathrm{Ag}$ Thin Films, Chem. Phys. Lett. 115 (1985) 130.

[111] S. Bernad, T. Soulimane and S. Lecomte, Redox and Conformational Equilibria of Cytochrome c552 from Thermus Thermophilus Adsorbed on a Chemically Modified Silver Electrode Probed by Surface-Enhanced Resonance Raman Spectroscopy, J. Raman Spectrosc. 35 (2003) 47.

[112] M. Roy, V.C. George, A.K. Dua, P. Raj, S. Schulze, D.A. Tenne, G. Salvan and D.R.T. Zahn, Detection of Nanophase at the Surface of HFCVD Grown Diamond Films using Surface Enhanced Raman Spectroscopic Technique, Diamond and Related Materials 11 (2002) 1858.

[113] D. Roy, Z.H. Barber and T.W. Clyne, Ag Nanoparticle Induced Surface Enhanced Raman Spectroscopy of Chemical Vapor Deposition Diamond Thin Films Prepared by Hot Filament Chemical Vapor Deposition, J. Appl. Phys. 91 (2002) 6085.

[114] J. Azoulay, A. Débarre, A. Richard, P. Tchénio, S. Bandow and S. Iijima, Polarised Raman Spectroscopy on a Single Class of Single-Wall Nanotubes by Nano SurfaceEnhanced Scattering, Chem. Phys. Lett. 331 (2000) 347.

[115] E.A. Ash and G. Nichols, Super-Resolution Aperture Scanning Microscope, Nature 237 (1972) 510.

[116] D.P. Tsai, A. Othonos and M. Moskovits, Raman Spectroscopy Using a Fiber Optic Probe with Subwavelength Aperture, Appl. Phys. Lett. 64 (1994) 1768. 
[117] B. Hecht, B. Sick, U.P. Wild, V. Deckert, R. Zenobi, O.J.F. Martin and D.W. Pohl., Scanning Near-Field Optical Microscopy with Aperture Probes: Fundamentals and Applications., J. Chem. Phys. 112 (18) (2000) 7761.

[118] D.W. Pohl, W. Denk and M. Lanz, Optical Spectroscopy: Image Recording with Resolution $\lambda / 20$., Appl. Phys. Lett. 44 (1984) 651.

[119] U. Dürig, D.W. Pohl and F. Rohner, Near-Field Optical-Scanning Microscopy, J. Appl. Phys. 59 (10) (1986) 3318.

[120] P.G. Gucciardi, S. Trusso, C. Vasi, S. Patane and M. Allegrini, Optical Near-Field Raman Imaging with Subdiffraction Resolution, Appl. Optics 42 (15) (2003) 2724.

[121] C.L. Jahncke, H.D. Hallen and M.A. Paesler, Nano-Raman Spectroscopy and Imaging with a Near-Field Scanning Optical Microscopy, J. Raman Spectrosc. 27 (1996) 579.

[122] H.D. Hallen and C.L. Jahncke, The Electric Field at the Apex of a Near-Field Probe : Implications for Nano-Raman Spectroscopy, J. Raman Spectrosc. 34 (2003) 655.

[123] D.A. Smith, S. Webster, M. Ayad, S.D. Evans, D. Fogherty and D. Batchelder, Development of a Scanning Near-Field Optical Probe for Localised Raman Spectroscopy, Ultramicroscopy 61 (1995) 247.

[124] S. Webster, D.A. Smith, D.N. Batchelder and S. Karlin, Sub-Micron Spatial Resolution Raman Spectroscopy and its Application to Stress Mapping in Silicon, Synthetic Metals 102 (1-3) (1999) 1425.

[125] A. Hartschuh, N. Anderson and L. Novotny, Near-Field Raman Spectroscopy using a Sharp Metal Tip, J. Microscopy 210 (Pt 3) (2003) 234.

[126] W.X. Sun and Z.X. Shen, A Practical Nanoscopic Raman Imaging Technique Realized by Near-Field Enhancement, Mater. Phys. Mech. 4 (2001) 17.

[127] W.X. Sun and Z.X. Shen, Near-Field Scanning Raman Microscopy using Apertureless Probes, J. Raman Spectrosc. 34 (2003) 668.

[128] D. Richards, R.G. Milner, F. Huang and F. Festy, Tip-Enhanced Raman Microscopy: Practicalities and Limitations, J. Raman Spectrosc. 34 (2003) 663.

[129] A. Hartschuh, M.R. Beversluis, A. Bouhelier and L. Novotny, Tip-Enhanced Optical Spectroscopy, Phil. Trans. Roy. Soc. Lond. A362 (2004) 807.

[130] B.E. Sernelius, Surface Modes in Physics, Wiley-VCH, Berlin (2001).

[131] B. Cao, W. Cai, G. Duan, Y. Li, Q. Zhao and D. Yu, A Template-free Electrochemical Deposition Route to ZnO Nanoneedle Arrays and their Optical and Field Emission Properties, Nanotechnology 16 (2005) 2567.

[132] K.N. Yu, Y. Xiong, Y. Liu and C. Xiong, Microstructural Change of Nano-SnO ${ }_{2}$ Grain Assemblages with the Annealing Temperature, Phys. Rev. B55 (1997) 2666.

[133] H.C. Choi, Y.M. Jung and S.B. Kim, Size Effects in the Raman Spectra of $\mathrm{TiO}_{2}$ Nanoparticles, Vibr. Spectrosc. 37 (2005) 33.

[134] A. Kailer, K.G. Nickel and Y.G. Gogotsi, Raman Microspectroscopy of Nanocrystalline and Amorphous Phases in Hardness Indentations, J. Raman Spectrosc. 30 (1999) 939.

[135] G.S. Cheng, S.H. Chen, X.G. Zhu, Y.Q. Mao and L.D. Zhang, Highly Ordered Nanostructures of Single Crystalline GaN Nanowires in Anodic Alumina Membranes, Mater. Sci. Eng. A286 (2000) 165.

[136] R.J.K. Wood, D.W. Wheeler, D.C. Lejeau and B.G. Mellor, Sand Erosion Performance of CVD Boron Carbide Coated Tungsten Carbide, Wear 233-235 (1999) 134.

[137] K.-H. Lee, H. Sugimura, Y. Inoue and O. Takai, Nano- and Micro-Tribological Characteristics of Amorphous Carbon Films Prepared by Shielded Arc Ion Plating, Thin Solid Films 435 (2003) 150. 
[138] V.V. Guliants, S.A. Holmes, J.B. Benziger, P. Heaney, D. Yates and I.E. Wachs, In Situ Studies of Atomic, Nano- and Macroscale Order during $\mathrm{VOHPO}_{4}, 0.5 \mathrm{H}_{2} \mathrm{O}$ transformation to $(\mathrm{VO}){ }_{2} \mathrm{P}_{2} \mathrm{O}_{7}$, J. Molec. Catal. A172 (2001) 265.

[139] H. Chen, X. Zhou and C. Ding, Investigation of the Thermomechanical Properties of a Plasma-Sprayed Nanostructured Zirconia Coating, J. Eur. Ceram. Soc. 23 (2003) 1449.

[140] S.-K. Lee, G.-J. Choi, U.-Y. Hwang, K.-K. Koo and T.-J. Park, Effect of Molar Ratio of $\mathrm{KOH}$ to Ti-isopropoxide on the Formation of BaTiO 3 Powders by Hydrothermal Method, Mater. Lett. 57 (2003) 2201.

[141] M.-S. Zhang, J. Yu, W.-C. Chen and Z. Yin, Optical and Structural Properties of Pure and Ce-doped Nanocrystals of Barium Titanate, Progr. Cryst. Growth Charact. Mater. (2000) 33.

[142] S.K. Tadokoro and E.N.S. Muccillo, Synthesis and Characterization of Nanosized Powders of Yttria-Doped Zirconia, J. Alloys and Compounds 344 (2002) 186.

[143] D.W. Zeng, C.S. Xie, B.L. Zhu and W.L. Song, Characteristics of $\mathrm{Sb}_{2} \mathrm{O}_{3}$ Nanoparticles Synthesised from Antimony by Vapor Condensation Method, Mater. Lett. 58 (3-4) (2004) 312.

[144] T. Zeng, Y. Qiu, L. Chen and X. Song, Microstructure and Phase Evolution of TiO Precursors Prepared by Peptisation-Hydrolysis Method using Polycarboxylic Acid as Peptizing Agent, Mater. Chem. Phys. 56 (1998) 163.

[145] P. Thangadurai, S. Ramasamy and R. Kesavamoorthy, Raman Studies in Nanocrystalline Lead (II) Fluoride, J. Phys.: Cond. Matter 17 (2005) 863.

[146] Y. Yang, C. Wang, J. Hou and J. Dai, Raman Scattering, Far Infrared Spectrum of BiI 3 Nanocrystallites, Mater. Lett. 57 (2003) 2185.

[147] P. Durán, F. Capel, J. Tartaj, D. Gutierrez and C. Moure, Heating-Rate Effect on the $\mathrm{BaTiO}_{3}$ Formation by Thermal Decomposition of Metal Citrate Polymeric Precursors, Solid State Ionics 141-142 (2001) 529.

[148]Z. Wang and S.K. Saxena, Raman Spectroscopic Study on Pressure-Induced Amorphization in Nanocrystalline Anatase ( $\left.\mathrm{TiO}_{2}\right)$, Solid State Comm. 118 (2001) 75.

[149] M. Boulova and G. Lucazeau, Crystallite NanoSize Effect on the Structural Transitions of $\mathrm{WO}_{3}$ Studied by Raman Spectroscopy, J. Solid State Chem. 167 (2002) 425.

[150] J.F. Meng, R.S. Katiyar and G.T. Zou, Grain Size Effect on Ferroelectric Phase Transition in $\mathrm{Pb}_{1-x} \mathrm{Ba}_{x} \mathrm{TiO}_{3}$ Ceramics, J. Phys. Chem. Solids 59 (6-7) (1998) 1161.

[151] K.C.V. Lima, A.G. de Souza Filho, A.P. Ayala, J.M. Filho, P.T.C. Freire, F.E.A. Melo, E.B. Araujo and J.A. Eiras, Raman Study of Morphotropic Phase Boundary in PbZr ${ }_{1-}$ ${ }_{x} \mathrm{Ti}_{x} \mathrm{O}_{3}$ at Low Temperatures, Phys. Rev. B63 (18) (2001) 41051.

[152] R.E. Melgarejo, M.S. Tomar, S. Bhaskar, P.S. Dobal and R.S. Katiyar, Large Ferroelectric Response in $\mathrm{Bi}_{4-x} \mathrm{Nd}_{x} \mathrm{Ti}_{3} \mathrm{O}_{12}$ Films Prepared by Sol-Gel Process, Appl. Phys. Lett. 81 (14) (2002) 2611.

[153] Z. Wang and S.K. Saxena, Pressure Induced Phase Transformations in Nanocrystalline Maghemite $\left(\gamma-\mathrm{Fe}_{2} \mathrm{O}_{3}\right)$, Solid State Comm. 123 (2002) 195.

[154] A. Trovarelli, F. Zamar, J. Llorca, C. de-Leitenburg, G. Dolcetti and J.T. Kiss, Nanophase Fluorite-Structured $\mathrm{CeO}_{2}-\mathrm{ZrO}_{2}$ Catalysts Prepared by High-energy Mechanical Milling, J. Catalysis 169 (1997) 490.

[155] E. Djurado, P. Bouvier and G. Lucazeau, Crystallite Size Effect on the TetragonalMonoclinic Transition of Undoped Nanocrystalline Zirconia Studied by XRD and Raman Spectrometry, J. Solid State Chem. 149 (2000) 399.

[156] P. Bouvier, J. Godlewski and G. Lucazeau, A Raman Study of the Nanocrystallite Size Effect on the Pressure-Temperature Phase Diagram of Zirconia Grown by Zirconiumbased Alloys Oxidation, J. Nucl. Mat. 300 (2002) 118. 
[157] P. Bouvier and G. Lucazeau, Raman Spectra and Vibrational Analysis of Nanometric Tetragonal Zirconia under High Pressure, J. Phys. Chem. Solids 61 (2000) 569.

[158] E. Barborini, I.N. Kholmanov, P. Piseri, C. Ducati, C.E. Bottani and P. Milani, Engineering the Nanocrystalline Structure of $\mathrm{TiO}_{2}$ Films by Aerodynamically Filtered Cluster Deposition, Appl. Phys. Lett. 81 (16) (2002) 3052.

[159] M.H. Frey and D.A. Payne, Grain-size Effect on Structure and Phase Transformations for Barium Titanate, Phys. Rev. B54 (1996) 3158.

[160] M.V. Abrashev, V.G. Ivanov, M.N. Iliev, R.A. Chakalov, R.I. Chakalova and C. Thomsen, Raman Study of the Variations of the Jahn-Teller Distortions through the MetalInsulator Transition in Magnetoresistive $\mathrm{La}_{0.7} \mathrm{Ca}_{0.3} \mathrm{MnO}_{3}$ Thin Films, phys. stat. sol. (b) 215 (1999) 631.

[161] R.C. Garvie, The Occurence of Metastable Tetragonal Zirconia as a Crystallite Size Effect, J. Phys. Chem. 69 (1965) 1238.

[162] T. Furukawa and W.B. White, Vibrational Spectra and Glass Structure, J. Non-Cryst. Sol. 38 \& 39 (1980) 87.

[163] Y. Wang, K. Tanaka, T. Nakaoka and K. Murase, Effect of Nanophase Separation on Crystallisation Process in Ge-Se Glasses Studied by Raman Scattering, Physica B 316-317 (2002) 568.

[164] M. Mortier, A. Monteville, G. Patriarche, G. Mazé and F. Auzel, New Progresses in Transparent Rare-Earth Doped Glass-Ceramics, Optical Materials 16 (2001) 255.

[165] Ph. Colomban, Raman Studies of Inorganic Gels and of their Sol-to-Gel, Gel-to-Glass and Glass-to-Ceramic Transformation, J. Raman Spectrosc. 27 (1996) 747.

[166] Ph. Colomban, Stress- and Nanostructure-Imaging of Ceramic Fibers and Abradable Thermal Barrier Coatings by Raman Microspectrometry : State of the Art and Perspectives, Ceram. Eng. \& Sci. Proc. 21 (2000) 143.

[167] A. Guinier, Théorie et Technique de la Radiocristallographie, Dunod, Paris (1956).

[168] Y. Guinet, T. Denicourt, A. Hedoux and M. Descamps, The Contribution of the Raman Spectroscopy to the Understanding of the Polyamorphism Situation in Triphenil Phosphite, J. Molec. Struct. 651-653 (2003) 507.

[169] D. Roy, M. Chhowalla, H. Wang, N. Sano, I. Alexandrou, T.W. Clyne and G.A.J. Amaratunga, Characterisation of Carbon Nano-Onions Using Raman Spectroscopy, Chem. Phys. Lett. 373 (2003) 52.

[170] J.-M. Herrera-Ramirez, Ph. Colomban and A. Bunsell, Micro-Raman Study of the Fatigue Fracture and Tensile Behaviour of Polyamide (PA 66) Fibres, J. Raman Spectrosc. 35 (12) (2004) 1063.

[171] A. Marcellan, A.R. Bunsell, R. Piques and Ph. Colomban, Micro-Mechanisms, Mechanical Behavior and Probabilistic Fracture Analysis of PA 66 Fibers, J. Mater. Sci. 38 (2003) 2117.

[172] A. Marcellan, Ph. Colomban and A. Bunsell, (Nano)structure, Internal Stress and in situ Fracture Behaviour of Polyamide Fibres, J. Raman Spectrosc. 35 (2004) 308.

[173] Ph. Colomban, J.M. Herrera-Ramirez, R. Paquin, A. Marcellan and A. Bunsell, MicroRaman Study of the Fatigue and Fracture Behaviour of Single PA66 Fibres. Comparison with Single PET and PP Fibres, Eng. Fract. Mech. 73 (16) (2006) 2463.

[174] B.H. Stuart, Polymer Crystallinity Studied using Raman Spectroscopy, Vibr. Spectrosc. 10 (1996) 79.

[175] C.C.C. Lesko, J.F. Rabolt, R.M. Ikeda, B. Chase and A. Kennedy, Experimental Determination of the Fiber Orientation Parameters and the Raman Tensor of the $1614 \mathrm{~cm}^{-}$ ${ }^{1}$ Band of Poly(ethylene terephtalate), J. Molec. Struct. 521 (2000) 127. 
[176] E. Bustarret, M.A. Hachicha and M. Brunel, Experimental Determination of the Nanocrystalline Volume Fraction in Silicon Thin Films from Raman Spectroscopy, Appl. Phys. Lett. 52 (20) (1988) 1675.

[177] M.F. Cerqueira, L. Rebouta, M. Andritschky, J.A. Ferreira and M.F. Da-Silva, Microcrystalline Silicon Thin Films Prepared by RF Reactive Magnetron Sputter Deposition, Vacuum 46 (12) (1995) 1385.

[178] D.G. Georgiev, M. Mitkova, P. Boolchand, G. Brunklaus, H. Eckert and M. Micoulaut, MolecularStructure, Glass Transition Temperature Variation, Agglomeration Theory, and Network Connectivity of Binary P-Se Glasses, Phys. Rev. B64 (2001) 134204.

[179] Y. Wang, J. Wells, D.G. Georgiev, P. Boolchand, K. Jackson and M. Micoulaut, Sharp Rigid to Floppy Phase Transition Induced by Dangling Ends in a Network Glass, Phys. Rev. Lett. 87 (18) (2001) 185503.

[180] H. Richter, Z.P. Wang and L. Ley, The One Phonon Raman Spectrum in Microcristalline Silicon, Solid State Comm. 39 (1981) 625.

[181] R. Carles, A. Mlayah, M.B. Amjoud, A. Reynes and R. Morancho, Structural Characterization of Ge Microcrystals in $G e_{x} C_{(1-x)}$ films, Jap. J. Appl. Phys. 31 (pt 1) (11) (1992) 3511.

[182] S. Rohmfeld, M. Hundhausen and L. Ley, Influence of Stacking Disorder on the Raman Spectrum of 3C-SiC, phys. stat. sol. (b) 215 (1999) 115.

[183] P. Parayanthal and F.H. Pollak, Raman Scattering in Alloy Semiconductors: "Spatial Correlation" Model, Phys. Rev. Lett. 52 (20) (1984) 1822.

[184] A. Fischer, L. Anthony and A.D. Compaan, Raman Analysis of Short-Range Clustering in Laser-Deposited CdS $S_{x} e_{1-x}$ Films, Appl. Phys. Lett. 72 (20) (1998) 2559.

[185] M. Fujii, S. Hayashi and K. Yamamoto, Growth of Ge Mycrocrystals in SiO $\mathrm{Shin}_{2}$ Film Matrices : A Raman and Electron Microscopic Study, Jap. J. Appl. Phys. 30 (1991) 687.

[186] W.F. Zhang, Y.L. He, M.S. Zhang, Z. Yin and Q. Chen, Raman Scattering Study on Anatase $\mathrm{TiO}_{2}$ Nanocrystals, J. Phys. D33 (2000) 912.

[187] J.W. Ager III, D.K. Veirs and G.M. Rosenblatt, Spatially Resolved Raman Studies of Diamond Films Grown by Chemical Vapor Deposition, Phys. Rev. B43 (1991) 6491.

[188] R.J. Nemanich, S.A. Solin and R.M. Martin, Light Scattering Study of Boron Nitride Microcrystals, Phys. Rev. B23 (12) (1981) 6348.

[189] A.C. Ferrari and J. Robertson, Interpretation of Raman Spectra of Disordered and Amorphous Carbon, Phys. Rev. B61 (20) (2000) 14095.

[190] S. Hofmann, C. Ducati, R.J. Neil, S. Piscanec, A.C. Ferrari, J. Geng, R.E. DuninBorkovski and J. Robertson, Gold Catalysed Growth of Silicon Nanowires by Plasma Enhanced Chemical Vapor Deposition, J. Appl. Phys. 94 (2003) 6005.

[191] Y.K. Kim and H.M. Jang, Raman Line-shape Analysis of Nano-Structural Evolution in Cation-Ordered $\mathrm{ZrTiO}_{4}$-Based Dielectrics, Solid State Comm. 127 (2003) 433.

[192] B. Li, D. Yu and S.-L. Zhang, Raman Spectral Study of Silicon Nanowires, Phys. Rev. B59 (1999) 1645.

[193] Q. Xiong, R. Gupta, K.W. Adu, E.C. Dickey, G.D. Lian, D. Tham, J.E. Fischer and P.C. Eklund, Raman Spectroscopy and Structure of Crystalline Gallium Phosphide Nanowires, J. Nanosci. Nanotech. 3 (2003) 335.

[194] I.H. Campbell and P.M. Fauchet, The Effects of Mycrocrystal Size and Shape on the One Phonon Raman Spectra of Crystalline Semiconductors, Solid State Comm. 58 (10) (1986) 739.

[195] J. Zi, H. Büscher, C. Falter, W. Ludwig, K. Zhang and X. Xie, Raman Shifts in Si Nanocrystals, Appl. Phys. Lett. 69 (1996) 200. 
[196] M.I. Vasilevskiy, A.G. Rolo and M.J.M. Gomes, One-Phonon Raman Scattering from Arrays of Semiconductor Nano-Crystals, Solid State Comm. 104 (1997) 381.

[197] I. Kosacki, T. Suzuki, V. Petrovsky, H.U. Anderson and Ph. Colomban, Raman Scattering and Lattice Defects in Nanocrystalline $\mathrm{CeO}_{2}$ Thin Films, Solid State Ionics 149 (1-2) (2002) 99.

[198] W.H. Weber, K.C. Hass and J.R. McBride, Raman Study of $\mathrm{CeO}_{2}$ : Second Order Scattering, Lattice Dynamics, and Particle-Size Effects, Phys. Rev. B48 (1993) 178.

[199] K.K. Tiong, P.M. Amirtharaj, F.H. Pollak and D.E. Aspnes, Effects of $\mathrm{As}^{+}$Ion Implantation on the Raman Spectra of GaAs: "Spatial Correlation" Interpretation, Appl. Phys. Lett. 44 (1984) 122.

[200] F. Tuinstra and J.L. Koenig, Raman Spectrum of Graphite, J. Chem. Phys. 53 (1970) 1126.

[201] F. Maury, R. Carles, G. Landa and B. Renucci, Raman Scattering Analysis of Disorder in Heterogeneous $(\mathrm{GaAs})_{1-x}\left(\mathrm{SiC}_{2}: \mathrm{H}\right)_{x}$ Films Grown by Metal-Organic Chemical Vapor Deposition, Thin Solid Films 155 (1987) 331.

[202] J. Zi, K. Zhang and X. Xie, Comparison of Models for Raman Spectra of Si Nanocrystals, Phys. Rev. B55 (15) (1997) 9263.

[203] G.D. Mahan, R. Gupta, Q. Xiong, C.K. Adu and P.C. Eklund, Optical Phonons in Polar Semiconductor Nanowires, Phys. Rev. B68 (2003) 073402.

[204] A. Tanaka, S. Onari and T. Arai, Raman Scattering from CdS Microcrystals Embedded in a Germanate Glass Matrix, Phys. Rev. B45 (12) (1992) 6587.

[205] V. Paillard, P. Puech, M.A. Laguna, R. Carles, B. Kohn and F. Huisken, Improved OnePhonon Confinement Model for an Accurate Size Determination of Silicon Nanocrystals, J. Appl. Phys. 86 (1999) 1921.

[206] J. Zuo, C. Xu, Y. Liu and Y. Quian, Crystallite Size Effects on the Raman Spectra of $\mathrm{Mn}_{3} \mathrm{O}_{4}$, Nanostruct. Mater. 10 (1998) 1331.

[207] R.S. Chen, C.C. Chen, Y.S. Huang, C.T. Chia, H.P. Chen, D.S. Tsai and K.K. Tiong, A Comparative Study of Microstructure of $\mathrm{RuO}_{2}$ Nanorods via Raman Scattering and Field Emission Scanning Electron Microscopy, Sol. State Comm. 131 (2004) 349.

[208] J. Zuo, C. Xu, B. Hou, C. Wang, Y. Xie and Y. Quian, Raman Spectra of Nanophase $\mathrm{Cr}_{2} \mathrm{O}_{3}$, J. Raman Spectrosc. 27 (1996) 921.

[209] S. Rohmfeld, M. Hundhausen and L. Ley, Raman Scattering in Polycrystalline 3C-SiC : Influence of Stacking Faults, Phys. Rev. B58 (15) (1998) 9858.

[210] M. Havel, PhD Thesis. Imageries Raman et Rayleigh de Nanophases. Nouveaux Outils pour l'Etude de la Corrosion de Matériaux Hétérogènes, University of Paris 6 - P. \& M. Curie (2004).

[211] L. Bergman, M.D. Bremser, W.G. Perry, R.F. Davis, M. Dutta and R.J. Nemanich, Raman Analysis of the Configurational Disorder in $A l_{x} G a_{1-x} N$ films, Appl. Phys. Lett. 71 (15) (1997).

[212] A.K. Arora, T.R. Ravindran, G.L.N. Reddy, A.K. Sikder and D.S. Misra, Nature of Confinement of Phonons in Nanocrystalline CVD Diamond, Diamond and Related Materials 10 (2001) 1477.

[213] M. Havel, D. Baron and Ph. Colomban, Smart Raman/Rayleigh Imaging of Nanosized SiC Materials Using the Spatial Correlation Model, J. Mater. Sci. 39 (20) (2004) 6183.

[214] M. Havel and Ph. Colomban, Skin/Bulk Nanostructure and Corrosion of SiC Based Fibres. A Surface Rayleigh and Raman Study, J. Raman Spectrosc. 34 (10) (2003) 786.

[215] M. Havel and Ph. Colomban, Raman and Rayleigh Mapping of Corrosion and Micromechanical Aging in SiC Fibres, Comp. Sci. Technol. 65 (3-4) (2005) 353. 
[216] J.E. Spanier, R.D. Robinson, F. Zhang, S.-W. Chan and I.P. Herman, Size-dependent Properties of $\mathrm{CeO}_{2-y}$ Nanoparticles as Studied by Raman Scattering, Phys. Rev. B64 (2001) 2450407.

[217] W.K. Choi, Y.W. Ho and V. Ng, Effect of Size of Ge Nanocrystals Embedded in $\mathrm{SiO}_{2}$ on Raman Spectra, Mater. Phys. Mech. 4 (2001) 46.

[218] M. Ivanda, K. Babocsi, C. Dem, M. Schmitt, M. Montagna and W. Kiefer, Low-wavenumber Raman Scattering from CdS $S_{x} S e_{1-x}$ Quantum Dots Embedded in a Glass Matrix, Phys. Rev. B67 (2003) 235329.

[219] A.V. Gomonnai, Y.M. Azhniuk, V.O. Yukhymchuk, M. Kranjcec and V.V. Lopushansky, Confinement-, Surface- and Disorder-related Effects in the Resonant Raman Spectra of Nanometric CdS $S_{1-x} S e_{x}$ Crystals, phys. stat. sol. (b) 239 (2003) 490.

[220] Y. Yan, S.-L. Zhang, S. Fan, W. Han, G. Meng and L. Zhang, Effect of Changing Incident Wavelength on Raman Features of Optical Phonons in SiC Nanorods and TaC Nanowires, Solid State Comm. 126 (2003) 649.

[221] L. Zhang, H.-J. Xie and C.-Y. Chen, Surface and Interface Optical Phonon Modes and Electron-Phonon Interaction in a Multi-shell Spherical System, Eur. Phys. J. B27 (2002) 577.

[222] Q. Xiong, J. Wang, O. Reese, L.C. Lew-Yan-Voon and P.C. Eklund, Raman Scattering from Surface Phonons in Rectangular Cross-sectional w-ZnS Nanowires, NanoLetters 4 (10) (2004) 1991.

[223] A. Roy and A.K. Sood, Surface and Confined Optical Phonons in $C d S_{x} S e_{1-x}$ Nanoparticles in a Glass Matrix, Phys. Rev. B53 (18) (1996) 12127.

[224] M.C. Klein, F. Hache, D. Ricard and C. Flytzanis, Size Dependence of Electron-Phonon Coupling in Semiconductor Nanospheres: the Case of CdSe, Phys. Rev. B42 (17) (1990) 11123.

[225] S. Hayashi and H. Kanamori, Raman Scattering from the Surface Phonon Mode in GaP Microcrystals, Phys. Rev. B26 (12) (1982) 7079.

[226] V.A. Fonoberov and A.A. Balandin, Polar Optical Phonons in Wurtzite Speroidal Quantum Dots: Theory and Application to $\mathrm{ZnO}$ and $\mathrm{ZnO} / \mathrm{MgZnO}$ Nanostructures, J. Phys.: Cond. Matter 17 (2005) 1085.

[227] R. Ruppin, Thermal Fluctuations and Raman Scattering in Small Spherical Crystals, J. Phys. C: Solid State Phys. 8 (1975) 1969.

[228] S. Hayashi and R. Ruppin, Raman Scattering from GaP Microcrystals: Analysis of the Surface Phonon Peak, J. Phys. C: Solid State Phys. 18 (1985) 2583.

[229] R. Ruppin and R. Englman, Optical Phonons of Small Crystals, Rep. Prog. Phys. 33 (1970) 149.

[230] J. Xu, G. Cheng, W. Yang and Y. Du, Raman Scattering from Surface Phonons in Fine Zn Particles Coated with ZnO, J. Phys. B: At. Mol. Opt. Phys. 29 (1996) 6227.

[231] F. Comas and C. Trallero-Giner, Surface Optical Phonons in Spherically Capped Quantum-Dot/Quantum-Well Heterostructures, J. Appl. Phys. 94 (2003) 6023.

[232] J.P. Sun, H.B. Teng, G.I. Haddad and M.A. Stroscio, Electron-interface Phonon Interaction in Multiple Quantum Well Structures, Semicond. Sci. Technol. 13 (1998) A147.

[233] N. Mori and T. Ando, Electron-optical-phonon Interaction in Single and Doble Heterostructures, Phys. Rev. B40 (1989) 6175.

[234] E. Roca, C. Trallero-Giner and M. Cardona, Polar Optical Vibrational Modes in Quantum Dots, Phys. Rev. B49 (19) (1994) 13704.

[235] T.D. Krauss, F.W. Wise and D.B. Tanner, Observation of Coupled Vibrational Modes of a Semiconductor nanocrystal, Phys. Rev. Lett. 76 (1996) 1376. 
[236] S. Nomura and T. Kobayashi, Exciton-LO Phonon Couplings in Spherical Semiconductor Microcrystallites, Phys. Rev. B45 (1992) 1305.

[237] E. Menéndez, C. Trallero-Giner and M. Cardona, Vibrational Resonant Raman Scattering in Spherical Quantum Dots: Exciton Effects, phys. stat. sol. (b) 199 (1997) 81.

[238] X. Hu and J. Zi, Reconstruction of Phonon Dispersion in Si Nanocrystals, J. Phys.: Cond. Matter 14 (2002) L671.

[239] D. Alexson, L. Bergman, M. Dutta, K.W. Kim, S. Komirenko, R.J. Nemanich, B.C. Lee, M.A. Stroscio and S. Yu, Confined Phonons and Phonon-mode Properties of III-V Nitrides with Wurtzite Crystal Structure, Physica B 263-264 (1999) 510.

[240] I.M. Tiginyanu, A. Sarua, G. Irmer, J. Monecke, S.M. Hubbard, D. Pavlidis and V. Valiaev, Frölich Modes in GaN Columnar Nanostructures, Phys. Rev. B64 (2001) 233317.

[241] Y.A. Pusep, M.T.O. Silva, J.C. Galzerani, N.T. Moshegov and P. Basmaji, Influence of Crystal-potential Fluctuations on Raman Spectra of Coupled Plasmon-LO-phonon Modes in Disordered Systems, Phys. Rev. B58 (16) (1998) 10683.

[242] M.I. Vasilevskiy, A.G. Rolo, M.J.M. Gomes, O.V. Vikhrova and C. Ricolleau, Impact of Disorder on Optical Phonons Confined in CdS Nano-Crystallites Embedded in a $\mathrm{SiO}_{2}$ Matrix, J. Phys.: Cond. Matter 13 (14) (2001) 3491.

[243] K. Ushizawa, M. N.-Gamo, K. Watanabe, I. Sakaguchi, Y. Sato and T. Ando, Raman Spectroscopic Study on $\{110\}$ Facet of Boron-Doped Chemical-Vapour-Deposited Diamond Crystals with Fano Line Fitting, J. Raman Spectrosc. 30 (1999) 957.

[244] P. Gonon, E. Gheeraert, A. Deneuville, F. Fontaine, L. Abello and G. Lucazeau, Characterization of Heavily B-doped Polycrystalline Diamond Films Using Raman Spectroscopy and Electron Spin Resonance, J. Appl. Phys. 78 (12) (1995) 7059.

[245] S. Nakashima and H. Harima, Raman Investigation of SiC Polytypes, phys. stat. sol. (a) 162 (39) (1997) 39.

[246] M. Park, V. Sakhrani, J.-P. Maria, J.J. Cuomo, C.W. Teng, J.F. Muth, M.E. Ware, B.J. Rodriguez and R.J. Nemanich, Wavelength-dependent Raman Scattering of Hydrogenated Amorphous Silicon Carbon with Red, Green, and Blue Light Excitation, J. Mater. Res. 18 (2003) 768.

[247] R. Meyer, L.J. Lewis, S. Prakash and P. Entel, Vibrational Properties of Nanoscale Materials: From Nanoparticles to Nanocrystalline Materials, Phys. Rev. B68 (2003) 104303.

[248] H. Lamb, On the Vibrations of an Elastic Sphere, Proc. London Math. Soc. 13 (1882) 189.

[249] H. Portalès, PhD Thesis. Etude par Diffusion Raman de Nanoparticules Métalliques en Matrice Diélectrique Amorphe, University of Lyon 1 - Claude Bernard (2001).

[250] A. Tamura, K. Higeta and T. Ichinokawa, Lattice Vibrations and Specific Heat of a Small Particle, J. Phys. C: Solid State Phys. 15 (1982) 4975.

[251] K.R. Patton and M.R. Geller, Phonons in a Nanoparticle Mechanically Coupled to a Substrate, Phys. Rev. B67 (2003) 155418.

[252] L. Saviot and D.B. Murray, The Connection between Elastic Scattering Cross Sections and Acoustic Vibrations of an Embedded Nanoparticle, phys. stat. sol. (c) 1 (11) (2004) 2634.

[253] J.A. Lobo, What Can we Learn about GW Physics with an Elastic Spherical Antenna?, Phys. Rev. D52 (1995) 591.

[254] D.A. Mazurenko, PhD Thesis. Ultrafast Optical Switching in Three-Dimensional Photonic Crystals ; Chapt 7: Coherent Vibrations of Submicron Gold Shells, University of Utrecht (2004). 
[255] A. Tanaka, S. Onari and T. Arai, Low-Frequency Raman Scattering from CdS Microcrystals Embedded in a Germanium Dioxide Glass Matrix, Phys. Rev. B47 (1993) 1237.

[256] M. Talati and P.K. Jha, Low-frequency Acoustic Phonons in Nanometric $\mathrm{CeO}_{2}$ Particles, Physica E 28 (2005) 171.

[257] P. Verma, W. Cordts, G. Irmer and J. Monecke, Acoustic Vibrations of Semiconductor Nanocrystals in Doped Glasses, Phys. Rev. B60 (1999) 5778.

[258] R.S. Cataliotti, G. Compagnini, A. Morresi, M. Ombelli and P. Sassi, Polarization Properties of Low Frequency Inelastic Scattering by Acoustic Phonons in Gold Nanoclusters, Chem. Chem. Phys. 4 (2002) 2774.

[259] E. Duval, L. Saviot, A. Mermet and D.B. Murray, Continuum Elastic Sphere Vibrations as a Model for Low Lying Optical Modes in ICosahedral Quasicrystals, J. Phys.: Cond. Matter 17 (2005) 3559.

[260] E. Duval, Far-Infrared and Raman Vibrational Transitions of a Solid Sphere: Selection Rules, Phys. Rev. B46 (1992) 5795.

[261] M. Montagna and R. Dusi, Raman Scattering from Small Spherical Particles, Phys. Rev. B52 (14) (1995) 10080.

[262] E. Duval, A. Boukenter and B. Champagnon, Vibration Eigenmodes and Size of Microcrystallites in Glass: Observation by Very-Low-Frequency Raman Scattering, Phys. Rev. Lett. 56 (19) (1986) 2052.

[263] A. Tamura and T. Ichinokawa, Frequency Spectrum of a Small Particle, J. Phys. C: Solid State Phys. 16 (1983) 4779.

[264] P. Gangopadhyay, R. Kesavamoorthy, K.G.M. Nair and R. Dhandapani, Raman Scattering Studies on Silver Nanoclusters in a Silica Matrix Formed by Ion-Beam Mixing, J. Appl. Phys. 88 (2000) 4975.

[265] M. Ferrari, F. Gonella, M. Montagna and C. Tosello, Detection and Size Determination of Ag Nanoclusters in Ion-Exchanged Soda-lime Glasses by Waveguided Raman Spectroscopy, J. Appl. Phys. 79 (1996) 2055.

[266] M. Ferrari, F. Gonella, M. Montagna and C. Tosello, Waveguide Raman Spectroscopy as a Tool for the Detection of Nanometric Metallic Particles in Glasses, J. Raman Spectrosc. 27 (1996) 793.

[267] R. Govindaraj, R. Kesavamoorthy, R. Mythili and B. Viswanathan, The Formation and Characterization of Silver Clusters in Zirconia, J. Appl. Phys. 90 (2001) 958.

[268] A. Gajovic, M. Stubicar, M. Ivanda and K. Furic, Raman Spectroscopy of Ball-Milled $\mathrm{TiO}_{2}$, J. Molec. Struct. 563-564 (2001) 315.

[269] A. Turkovic, M. Ivanda, S. Popovic, A. Tonejc, M. Gotic, P. Dubcek and S. Music, Comparative Raman, XRD, HREM and SAXS Studies of Grain Sizes in Nanophase TiO2, J. Molec. Struct. 410-411 (1997) 271.

[270] M. Gotic, M. Ivanda, A. Sekulic, S. Music, S. Popovic, A. Turkovic and K. Furic, Microstructure of Nanosized $\mathrm{TiO}_{2}$ Obtained by Sol-Gel Synthesis, Mater. Lett. 28 (1996) 225.

[271] M. Fujii, Y. Kanzawa, S. Hayashi and K. Yamamoto, Raman Scattering from Acoustic Phonons Confined in Si Nanocrystals, Phys. Rev. B54 (12) (1996) R8373.

[272] F. Liu, L. Liao, G. Wang, G. Cheng and X. Bao, Experimental Observation of Surface Modes of Quasifree Clusters, Phys. Rev. Lett. 76 (1996) 604.

[273] M. Ikezawa, T. Okuno, Y. Masumoto and A.A. Lipovskii, Complementary Detection of Confined Acoustic Phonons in Quantum Dots by Coherent Phonon Measurement and Raman Scattering, Phys. Rev. B64 (2001) 201315. 
[274] T.D. Krauss and F.W. Wise, Coherent Acoustic Phonons in a Semiconductor Quantum Dot, Phys. Rev. Lett. 79 (25) (1997) 5102.

[275] G. Mariotto, M. Montagna, G. Viliani, E. Duval, S. Lefrant, E. Rzepka and C. Mai, Low-energy Raman Scattering from Silver Particles in Alkali Halides, EuroPhys. Lett. 6 (1988) 239.

[276] J. Hernandez-Rosas, M. Picquart, E. Haro-Poniatowski, M. Kanehisa, M. Jouanne and J.F. Morhange, Elastic Vibrations of Spheroidal Nanometric Particles, J. Phys.: Cond. Matter 15 (2003) 7481.

[277] G. Irmer, J. Monecke, P. Verma, G. Goerigk and M. Herms, Size Analysis of Nanocrystals in Semiconductor Doped Silicate Glasses with Anomalous Small-angle x ray and Raman Scattering, J. Appl. Phys. 88 (2000) 1873.

[278] A. Roy and A.K. Sood, Growth of CdS $S_{x} S e_{1-x}$ Nanoparticles in Glass Matrix by Isochronal Thermal Annealing: Confines Acoustic Phonons and Optical Absorptions Studies, Solid State Comm. 97 (1996) 97.

[279] D.B. Murray and L. Saviot, Phonons in Inhomogeneous Continuum: Vibrations of an Embedded Nanoparticle, Phys. Rev. B69 (2004) 094305.

[280] L. Saviot, D.B. Murray and M.d.C. Marco de Lucas, Vibrations of Free and Embedded Anisotropic Elastic Spheres: Application to Low-Frequency Raman Scattering of Silicon Nanoparticles in Silica, Phys. Rev. B69 (2004) 113402.

[281] N.N. Ovsyuk and V.N. Novikov, Influence of a Glass Matrix on Acoustic Phonons Confines in Microcrystals, Phys. Rev. B53 (1996) 3113.

[282] G. Bachelier and A. Mlayah, Surface Plasmon Mediated Raman Scattering in Metal Nanoparticles, Phys. Rev. B69 (2004) 205408.

[283] O.L. Lazarenkova and A.A. Balandin, Raman Scattering from Three-dimensionally Regimented Quantum Dots Superlattices, Superlattices and Microstructures 33 (2003) 95.

[284] M. Rajalakshmi and A.K. Arora, Vibrational Spectra of Selenium Nanoparticles Dispersed in a Polymer, Nanostruct. Mater. 11 (1999) 399.

[285] P. Nandakumar, C. Vijayan, M. Rajalakshmi, A.K. Arora and Y.V.G.S. Murti, Raman Spectra of CdS Nanocrystals in Nafion: Longitudinal Optical and Confined Acoustic Phonons Modes, Physica E 11 (2001) 377.

[286] D. Shuo, L. Jin-Quan and L. Yu-Long, Enhanced Raman Scattering from Nano-SnO Grains, Chinese Phys. 13 (11) (2004) 1854.

[287] G.G. Siu, M.J. Stokes and Y. Liu, Variation of Fundamental and Higher-order Raman Spectra of $\mathrm{ZrO}_{2}$ Nanograins with Annealing Temperature, Phys. Rev. B59 (1999) 3173.

[288] Y.M. Yang, X.L. Wu, L.W. Yang, G.S. Huang, G.G. Siu and P.K. Chu, Low-frequency Raman Scattering of Ge and Si Nanocrystals in Silica Matrix, J. Appl. Phys. 98 (2005) 064303.

[289] J.P. Wittmer, A. Tanguy, J.-L. Barrat and L. Lewis, Vibrations of Amorphous, Nanometric Structures: When Does Continuum Theory Apply?, Europhys. Lett. 57 (2002) 423.

[290] M. Fujii, T. Nagareda, S. Hayashi and K. Yamamoto, Low-frequency Raman Scattering from Silver Particles Embedded in SiO 2 Thin Films, Phys. Rev. B 44 (12) (1991) 6243.

[291] C.Q. Sun, T.P. Chen, B.K. Tay, S. Li, H. Huang, Y.B. Zhang, L.K. Pan, S.P. Lau and X.W. Sun, An Extended "Quantum Confinement" Theory: Surface-coordination Imperfection Modifies the Entire Band Structure of a Nanosolid, J. Phys. D: Appl. Phys. 34 (2001) 3470.

[292] M.S. Benrakkad, M.A. Benitez, J. Esteve, J.M. Lopez-Villegas, J. Samitier and J.R. Morante, Stress Measurement by Micro-Raman Spectroscopy of Polycrystalline Silicon Structures, J. Micromech. Microeng. 5 (1995) 132. 
[293] K.A. Alim, V.A. Fonoberov and A.A. Balandin, Origin of the Optical Phonon Frequency Shifts in ZnO Quantum Dots, Appl. Phys. Lett. 86 (2005) 053103.

[294] L.A. Falkovsky and J. Camassel, Disorder Effect on Optical Phonons, Physica B 284288 (2000) 1145.

[295] S. Karlin and Ph. Colomban, Phase Diagram, Short Range Structure and Amorphous Phases in the $\mathrm{ZrO}_{2}-\mathrm{GeO}_{2}\left(-\mathrm{H}_{2} \mathrm{O}\right)$ System, J. Am. Ceram. Soc. 82 (1999) 735.

[296] F. Touati, N. Gharbi and Ph. Colomban, Structural Evolution in Polyolysed Hybrid Organic-Inorganic Alumina Gels, J. Mater. Sci. 35 (2000) 1565.

[297] Ph. Colomban, H. Courret, F. Romain, G. Gouadec and D. Michel, Sol-Gel Prepared Pure and Li-doped Hexacelsian Polymorphs : An IR, Raman and Thermal Expansion Study of the $\beta$ Phase Stabilization by Frozen Short-Range Disorder, J. Am. Ceram. Soc. 83 (12) (2000) 2974.

[298] A. Kremenovic, Ph. Colomban, B. Piriou, D. Massiot and P. Florian, Structure and Spectroscopic Characterization of the Quenched Hexacelsian, J. Phys. Chem. Solids 64 (11) (2003) 2253.

[299] D. Michel, Ph. Colomban, S. Abolhassani, F. Voyron and A. Kahn-Harari, Germanium Mullite : Structure and Vibrational Spectra of Gels, Glasses and Ceramics, J. Eur. Ceram. Soc. 16 (1996) 161.

[300] Ph. Colomban, F. Romain, A. Neiman and I. Animitsa, Double Perovskites with Oxygen Structural Vacancies: Raman Spectra, Conductivity and Water Uptake, Solid State Ionics 145 (2001) 339.

[301] R. Dimitrijevic, A. Kremenovic, V. Dondur, M. Tomasevic-Canovic and M. Mitrovic, Thermally Induced Conversion of Sr-exchanged LTA- and FAU-Framework Zeolites. Synthesis, Characterization and Polymorphism of Ordered and Disordered $\mathrm{Sr}_{1_{-x}} A l_{2-}$ ${ }_{2 x} \mathrm{Si}_{2+2 x} \mathrm{O}_{8}(x=0,0.15)$ Diphyllosilicate and Feldspar Phase, J. Phys. Chem. B101 (1997) 3931.

[302] R.A. Barrio, F.L. Galeener, E. Martinez and R.J. Elliott, Regular Ring Dynamics in AX Tetrahedral Glasses, Phys. Rev. B48 (21) (1993) 15672.

[303] A. Pasquarello and R. Car, Identification of Raman Defect Lines as Signatures of Ring Structures in Vitreous Silica, Phys. Rev. Lett. 80 (23) (1998) 5145.

[304] T. Uchino, Y. Kitagawa and T. Yoko, Structure, Energies, and Vibrational Properties of Silica Rings in $\mathrm{SiO}_{2}$ Glass, Phys. Rev. B61 (2000) 234.

[305] A. Rahmani, M. Benoit and C. Benoit, Signature of Small Rings in the Raman Spectra of Normal and Compressed Amorphous Silica: A Combined Classical and ab initio Study, Phys. Rev. B68 (2003) 184202.

[306] Ph. Colomban, Polymerization Degree and Raman Identification of Ancient Glasses used for Jewelry, Ceramic Enamels and Mosaics, J. Non-Cryst. Sol. 323 (1-3) (2003) 180.

[307] Ph. Colomban and O. Paulsen, Raman Determination of the Structure and Composition of Glazes, J. Am. Ceram. Soc. 88 (2005) 390.

[308] F.A. Seifert, B.O. Mysen and D. Virgo, Three-Dimensional Network Structure of Quenched Melts (Glass) in the Systems $\mathrm{SiO}_{2}-\mathrm{NaAlO}_{2}, \mathrm{SiO}_{2}-\mathrm{CaAl}_{2} \mathrm{O}_{4}$ and $\mathrm{SiO}_{2}$ $\mathrm{MgAl}_{2} \mathrm{O}_{4}$, Am. Miner. 67 (1982) 696.

[309] R. Shuker and R.W. Gammon, Raman-scattering Selection-Rule Breaking and the Density of States in Amorphous Materials, Phys. Rev. Lett. 25 (1970) 222.

[310] C. Masciovecchio, V. Mazzacurati, G. Monaco, G. Ruocco, T. Scopigno, F. Sette, P. Benassi, A. Cunsolo, A. Fontana, M. Krisch, A. Mermet, M. Montagna, F. Rossi, M. Sampoli, G. Signorelli and R. Verbeni, Acoustic Nature of the Boson Peak in Vitreous Silica, Phil. Mag. Pt B, Phys. Cond. Matter 79 ((11-12)) (1999) 2013. 
[311] M.J. Harris, M.T. Dove and J.M. Parker, Floppy Modes and the Boson Peak in Crystalline and Amorphous Silicates; an Inelastic Neutron Scattering Study, Miner. Mag. 64 (2000) 435.

[312] M.J. Harris, S.M. Bennington, M.T. Dove and J.M. Parker, On the Wave Vector Dependance of the Boson Peak in Silicate Glasses and Crystals, Physica B 263-264 (1999) 357.

[313] G. Mariotto, M. Montagna, G. Viliani, R. Campostrini and G. Carturan, Low-Frequency Raman Scattering in Thermally Treated Silica Gels: Observation of Phonon-Fracton Crossover, J. Phys. C: Solid State Phys. 21 (1988) L797.

[314] N.V. Surovtsev and A.P. Sokolov, Inhomogeneous, Disordered, and Partially Ordered Systems - Frequency Behavior of Raman Coupling Coefficient in Glasses, Phys. Rev. B66 (2002) 54205.

[315] A. Chmel and V.S. Shashkin, Observation of the Raman Scattering by Acoustic Vibrations of Fractal Voids, Europhys. Lett. 55 (2001) 235.

[316] A. Roy and A.K. Sood, Fracton Dimension of Porous Silicon as Determined by LowFrequency Raman Scattering, Solid State Comm. 93 (12) (1995) 995.

[317] A. Boukenter, B. Champagnon, E. Duval, J.L. Rousset, J. Dumas and J. Serughetti, Vibrational Modes in Silica Aerogels: Low-Frequency Raman Scattering, J. Phys. C: Solid State Phys. 21 (1988) L1097.

[318] E. Courtens and R. Vacher, Experiments on the Structure and Vibrations of Fractal Solids, Proc. Royal Soc. London A423 (1864) (1989) 55.

[319] E. Duval, A. Boukenter and T. Achibat, Vibrational Dynamics and the Structure of Glasses, J. Phys.: Cond. Matter 2 (1990) 10227.

[320] A.J. Martin and W. Brenig, Model for Brillouin Scattering in Amorphous Solids, phys. stat. sol. (b) 64 (1974) 163.

[321] N.V. Surotsev, Features of the Raman Coupling Coefficient of Boson Peak Vibrations in Glasses, phys. stat. sol. (c) 1 (11) (2004) 2867.

[322] V.L. Gurevich, D.A. Parshin and H.R. Schober, Anharmonicity, Vibrational Instability, and the Boson Peak in Glasses, Phys. Rev. B67 (2003) 094203.

[323] C. Boulesteix, K.C. Hewitt and J.C. Irwin, Temperature-Induced Frequency Shift of the Raman-active $\mathrm{CuO}_{2}$ Planar Oxygen Vibrational Modes of Bi-2212 Related to a Change of the Cu-O Bonding, J. Phys.: Cond. Matter 12 (2000) 9637.

[324] P. Li, W. Yang, P. Tan, H. Wen and Z. Zhao, Raman-Forbidden Mode and Oxygen Ordering in $\mathrm{Bi}_{2} \mathrm{Sr}_{2-x} \mathrm{La}_{x} \mathrm{CuO}_{6+y}$ Single Crystals Annealed in Oxygen, Phys. Rev. B61 (17) (2000) 11324.

[325] K.C. Hewitt, N.L. Wang, J.C. Irwin, D.M. Pooke, A.E. Pantoja and H.J. Trodahl, Isotope Shift of the 590-cm ${ }^{-1}$ Raman Feature in Underdoped $\mathrm{Bi}_{2} \mathrm{Sr}_{2} \mathrm{CaCu}_{2} \mathrm{O}_{8+\delta}$, (1999).

[326] A. Gruger, A. Regis, T. Schmatko and Ph. Colomban, Nanostructure of Nafion Membranes at Different States of Hydration. An IR and Raman Study, Vibr. Spectrosc. 26 (215-225) (2001).

[327] I.G. Siny, R.S. Katiyar and A.S. Bhalla, Cation Arrangement in the Complex Perovskites and Vibrational Spectra, J. Raman Spectrosc. 29 (1998) 358.

[328] T. Runka, R. Aleksiyko, M. Berkowski and M. Drozdowski, Raman Scattering Study of $\left(\mathrm{SrAl}_{0.5} \mathrm{Ta}_{0.5} \mathrm{O}_{3}\right)_{1-x-y}:\left(\mathrm{LaAlO}_{3}\right)_{x}:\left(\mathrm{CaAl}_{0.5} \mathrm{Ta}_{0.5} \mathrm{O}_{3}\right)_{y}$ Solid Solution Crystals, Cryst. Res. Technol. 40 (4/5) (2005) 453.

[329] J.-P. Boilot, Ph. Colomban, R. Collongues, G. Collin and R. Conoes, X-Ray Scattering Evidence of Sublattice Phase Transition in Stoichiometric Silver $\beta$-Alumina, Phys. Rev. Lett. 42 (12) (1979) 785. 
[330] R. Collongues, D. Gourier, A. Kahn, J.P. Boilot, Ph. Colomban and A. Wicker, $\beta$ Alumina, a Typical Solid Electrolyte : Latest Developments in Fundamental Approach and in Battery Utilization, J. Phys. Chem. Sol. 45 (10) (1984) 981.

[331] Ph. Colomban, F. Fillaux, J. Thomkinson and G.J. Kearley, Inelastic NeutronScattering Study of Proton Dynamics in $\beta$-alumina, Solid State Ionics 77 (1995) 45.

[332] S.A. Solin, Lattice Dynamics II. Optical Studies, In: Graphite Intercalation Compounds I. Structure and Dynamics (Chap 5). Eds. H. Zabel and S.A. Solin, Springer-Verlag, 157 (1990).

[333] C. Thomsen and S. Reich, Double Resonant Raman Scattering in Graphite, Phys. Rev. Lett. 85 (24) (2000) 5214.

[334] M. Havel and Ph. Colomban, Rayleigh and Raman Images of the Bulk/Surface Nanostructure of SiC Based Fibres, Composites B35 (2004) 139.

[335] E. Tomasella, L. Thomas, C. Meunier, M. Nadal and S. Mikhailov, Coupled Effects of Bombarding Ions Energy on the Microstructure and Stress Level of RFPECVD a-C:H Films: Correlation with Raman Spectroscopy, Surf. Coat. Technol. 174-175 (2003) 360.

[336] E. Tomasella, C. Meunier and S. Mikhailov, a-C:H Films Deposited by RadioFrequency Plasma: Influence of Gas Composition on Structure, Optical Properties and Stress Levels, Surf. Coat. Technol. 141 (2001) 286.

[337] A.H. Jayatissa, F. Sato, N. Saito, H. Ohnishi, K. Takizawa, Y. Nakanishi and T. Yamaguchi, Structural Properties of Carbon Films Deposited by Pulsed ArF Laser Ablation: Effects of Substrate Temperature, Bias and $\mathrm{H}_{2}$ Pressure, Mater. Sci. Eng. B55 (1-2) (1998) 143.

[338] W.-Y. Wu and J.-M. Ting, Growth and Characteristics of Carbon Films with NanoSized Metal Particles, Thin Solid Films 420-421 (2002) 166.

[339] K.W.R. Gilkes, H.S. Sands, D.N. Batchelder, J. Robertson and W.I. Milne, Direct Observation of $\mathrm{sp}^{3}$ Bonding in Tetrahedral Amorphous Carbon Using UV Raman Spectroscopy, Appl. Phys. Lett. 70 (15) (1997) 1980.

[340] M. Yoshikawa, G. Katagiri, H. Ishida, A. Ishitani and T. Akamatsu, Resonant Raman Scattering of Diamond-Like Amorphous Carbon Films, Appl. Phys. Lett. 52 (19) (1988) 1639.

[341] M. Yoshikawa, G. Katagiri, H. Ishida, A. Ishitani and T. Akamatsu, Raman Spectra of Diamond-Like Amorphous Carbon Films, Solid State Comm. 66 (11) (1988) 1177.

[342] H.-J. Scheibe, D. Drescher and P. Alers, Raman Characterization of Amorphous Carbon Films, Fresenius J. Anal. Chem. 353 (1995) 695.

[343] M. Park, S.M. Camphausen, A.F. Myers, P.T. Barletta, V. Sakhrani, L. Bergman, R.J. Nemanich and J.J. Cuomo, Raman Scattering of Tetrahedrally-Bonded Amorphous Carbon Deposited at Oblique Angles, Mater. Lett. 41 (1999) 229.

[344] C. Fauteux, R. Longtin, J. Pegna and M. Boman, Microstructure and Growth Mechanism of Laser Grown Carbon Microrods as a function of Experimental Parameters, J. Appl. Phys. 95 (2004) 2737.

[345] R.C. Mani, S. Sharma, M.K. Sunkara, J. Gullapalli, R.P. Baldwin, R. Rao, A.M. Rao and J.M. Cowley, Synthesis and Electrochemical Characteristics of a Nanocomposite Diamond Electrode, Electrochem. and Solid State Lett. 5 (2002) E32.

[346] B.K. Tay, X. Shi, H.S. Tan, H.S. Yang and Z. Sun, Raman Studies of Tetrahedral Amorphous Carbon Films Deposited by Filtered Cathodic Vacuum Arc, Surf. Coat. Technol. 105 (1998) 155.

[347] H.W. Kroto, J.R. Heath, S.C. O'Brien, R.F. Curl and R.E. Smalley, C60: Buckminsterfullerene, Nature 318 (6042) (1985) 162. 
[348] J.F. Maguire, M.S. Amer and J. Busbee, Exploring Two-Dimensional Soap-Foam Films using Fullerene $\left(C_{60}\right)$ Nanosensors, Appl. Phys. Lett. 82 (16) (2003) 2592.

[349] S. Iijima, Helical microtubules of graphitic carbon, Nature 354 (1991) 56.

[350] R.H. Baughman, A.A. Zakhidov and W.A. de Heer, Carbon Nanotubes - the Route Toward Applications, Science 297 (5582) (2002) 787.

[351] C. Thomsen and S. Reich, Raman Scattering in Carbon Nanotubes, In: Light Scattering in Solids IX : Novel Materials and Techniques (Chap. 3). Eds. M. Cardona and R. Merlin, Springer, Heidelberg, (2006).

[352] M.S. Dresselhaus, G. Dresselhaus, R. Saito and A. Jorio, Raman Spectroscopy of Carbon Nanotubes, Physics Reports 409 (2005) 47.

[353] P.C. Eklund, J.M. Holden and R.A. Jishi, Vibrational Modes of Carbon Nanotubes ; Spectroscopy and Theory, Carbon 33 (1995) 959.

[354] E. Richter and K.R. Subbaswamy, Theory of Size-Dependant Resonance Raman Scattering from Carbon Nanotubes, Phys. Rev. Lett. 79 (14) (1997) 2738.

[355] J. Kürti, G. Kresse and H. Kuzmany, First-Principles Calculations of the Radial Breathing Mode of Single-Wall Carbon Nanotubes, Phys. Rev. B58 (14) (1998) 8869.

[356] S. Bandow, S. Asaka, Y. Saito, A.M. Rao, L. Grigorian, E. Richter and P.C. Eklund, Effect of the Growth Temperature on the Diameter Distribution and Chirality of SingleWall Carbon Nanotubes, Phys. Rev. Lett. 80 (17) (1998) 3779.

[357] H. Kuzmany, W. Plank, M. Hulman, C. Kramberger, A. Grüneis, T. Pichler, H. Peterlik, H. Kataura and Y. Achiba, Determination of SWCNT Diameters from the Raman Response of the Radial Breathing Mode, Eur. Phys. J. B22 (2001) 307.

[358] R. Saito, T. Takeya, T. Kimura, G. Dresselhaus and M.S. Dresselhaus, Raman Intensity of Single-Wall Carbon Nanotubes, Phys. Rev. B57 (1998) 4145.

[359] A.G. de Souza Filho, A. Jorio, G.G. Samsonidze, G. Dresselhaus, M.S. Dresselhaus, A.K. Swan, M.S. Unlu, B.B. Goldberg, R. Saito, J.H. Hafner, C.M. Lieber and M.A. Pimenta, Probing the Electronic Trigonal Warping Effect in Individual Single-Wall Carbon Nanotubes using Phonon Spectra, Chem. Phys. Lett. 354 (2002) 62.

[360] M.A. Pimenta, A. Marucci, S.D.M. Brown, M.J. Matthews, A.M. Rao, P.C. Eklund, R.E. Smalley, G. Dresselhaus and M.S. Dresselhaus, Resonant Raman Effect in SingleWall Carbon Nanotubes, J. Mater. Res. 13 (1998) 2396.

[361] M.S. Dresselhaus, G. Dresselhaus, A. Jorio, A.G. de Souza Filho and R. Saito, Raman Spectroscopy of Isolated Single Wall Carbon Nanotubes, Carbon 40 (2002) 2043.

[362] T.W. Odom, J.-L. Huang, P. Kim, M. Ouyang and C.M. Lieber, Scanning Tunneling Microscopy and Spectroscopy Studies Of Single Wall Carbon Nanotubes, J. Mater. Res. 13 (1998) 2380.

[363] M. Hulman, R. Pfeiffer and H. Kuzmany, Raman Spectroscopy of Small-Diameter Nanotubes, New J. Phys. 6 (2004) 1.

[364] M.A. Pimenta, E.B. Hanlon, A. Marucci, P. Corio, S.D.M. Brown, S.A. Empedocles, M. Bawendi, G. Dresselhaus and M.S. Dresselhaus, The Anomalous Dispersion of the Disorder-Induced and the Second-order Raman Bands in Carbon Nanotubes, Braz. J. Phys. 30 (2000) 423.

[365] V.N. Popov and P. Lambin, Radius and Chirality Dependence of the Radial Breathing Mode and the G-Band Phonon Modes of Single-Walled Carbon Nanotubes, Phys. Rev. B73 (2006) 085407.

[366] K.K. Chawla, Composite Materials Science and Engineering, $2^{\text {nd }}$ edition, SpringerVerlag, New York (1998).

[367] G. Salvador and W.F. Sherman, Pressure Dependance of the Raman Phonon Spectrum in 6H-Silicon Carbide, J. Molec. Struct. 247 (1991) 373. 
[368] W.J. Choyke and G. Pensl, Physical Properties of SiC, MRS Bull. 22 (1997) 25.

[369] D.W. Feldman, J.H. Parker, W.J. Choyke and L. Patrick, Raman Scattering in 6H-SiC, Phys. Rev. 170 (1968) 698.

[370] M. Takeda, J. Sakamoto, A. Saeki, Y. Imai and H. Ichikawa, High Performance Silicon Carbide Fibre Hi-Nicalon for CMCs, Ceram. Eng. \& Sci. Proc. 16 (1995) 37.

[371] K. Kumagawa, H. Yamaoka, M. Shibuya and T. Yamamura, Fabrication and Mechanical Properties of New Improved Si-M-C-(O) Tyranno Fiber, Ceram. Eng. \& Sci. Proc. 19 (1998) 65.

[372] Dow Corning Corp., Information about Sylramic ${ }^{T M}$ SiC Fiber, Product Inform. Form No.10-754-97 (1997).

[373] S. Karlin and Ph. Colomban, Raman Study of the Chemical and Thermal Degradation of As-Received and Sol-Gel Embedded Nicalon and Hi-Nicalon SiC Fibres used in Ceramic Matrix Composites, J. Raman Spectrosc. 28 (1997) 219.

[374] S. Karlin and Ph. Colomban, Micro Raman Study of SiC Fibre - Oxide Matrix Reaction, Composites B29 (1998) 41.

[375] H. Okumura, E. Sakuma, J.H. Lee, H. Mukaida, S. Misawa, K. Endo and S. Yoshida, Raman Scattering of SiC : Application to the Identification of Heteroepitaxy of SiC Polytypes, J. Appl. Phys. 61 (1987) 1134.

[376] Y. Sasaki, Y. Nishina, M. Sato and K. Okamura, Raman Study of SiC Fibres Made from PCS, J. Mater. Sci. 22 (1987) 443.

[377] S.-I. Nakashima, Y. Nakakura and I. Zenzaburo, Structural Identification of SiC Polytypes by Raman Scattering : 27R and 33R Polytypes., J. Phys. Soc. Jap. 56 (1987) 359.

[378] Y.G. Gogotsi, K.G. Nickel, D. Bahloul-Hourlier, T. Merle-Mejean, G.E. Khomenko and K.P. Skjerlie, Structure of Carbon Produced by Hydrothermal Treatment of $\beta$-SiC Powder, J. Mater. Chem. 6 (1996) 595.

[379] G. Gouadec, PhD Thesis. Analyse (Micro)-Mécanique et (Nano)-Structurale de Solides Hétérogènes par Spectroscopie Raman, University of Rennes 1 (2001).

[380] M.-H. Berger, N. Hochet and A.R. Bunsell, Properties and Microstructures of Small Diameter SiC-Based Fibers, In: Fine Ceramic Fibers (Chap. 6). Eds. A.R. Bunsell and M.-H. Berger, Marcel Dekker Inc., New York, 207 (1999).

[381] J. Lipowitz, J.A. Rabe, K.T. Nguyen, L.D. Orr and R.R. Androl, Structure and Properties of Polymer-Derived Stoichiometric Fiber, Ceram. Eng. \& Sci. Proc. 16 (1995) 55.

[382] M. Takeda, J. Sakamoto, Y. Imai, H. Ichikawa and T. Ishikawa, Properties of Stoichiometric Silicon Carbide Fibre Derived from PCS, Ceram. Eng. \& Sci. Proc. 15 (1994) 133.

[383] T. Ishikawa, Y. Kohtoku, K. Kumagawa, T. Yamamura and T. Nagasawa, HighStrength Alkali-Resistant Sintered SiC Fibre Stable to 2,200 C, Nature 391 (6669) (1998) 773.

[384] M.-H. Berger, N. Hochet and A.R. Bunsell, Microstructure and High Temperature Mechanical Behavior of New Polymer Derived SiC Based Fibres, Ceram. Eng. \& Sci. Proc. 19 (1998) 39.

[385] D.W. Feldman, J.H. Parker, W.J. Choyke and L. Patrick, Phonon Dispersion Curves by Raman Scattering in SiC, Polytypes 3C, 4H, 6H, 15R and 21R, Phys. Rev. 173 (1968) 787.

[386] I. Kosacki, T. Suzuki, V. Petrovsky, H.U. Anderson and Ph. Colomban, Lattice Defects in Nanocrystalline $\mathrm{CeO}_{2}$ Thin Films, Rad. Eff. Def. Sol. 156 (2001) 109.

[387] I. Kosacki, V. Petrovsky, H.U. Anderson and Ph. Colomban, Raman Spectroscopy of Nanocrystalline Ceria and Zirconia Thin Films, J. Am. Ceram. Soc. 85 (11) (2002) 2646. 
[388] R.J. Young, Analysis of Composites Using Raman and Fluorescence Microscopy - a Review, J. Microscopy 185 (1996) 199.

[389] C. Vlattas and C. Galiotis, Monitoring the Behavior of Polymer Fibres Under Axial Compression, Polymer 32 (10) (1991) 1788.

[390] C. Galiotis, Laser Raman Spectroscopy, a New Stress/Strain Measurement Technique for the Remote and On-Line Nondestructive Inspection of Fiber Reinforced Polymer Composites, Mater. Technol. 8 (9/10) (1993) 203.

[391] I.J. Beyerlein, M.S. Amer, L.S. Schadler and S.L. Phoenix, New Methodology for Determining in-situ Fibre, Matrix and Interfaces Stresses in Damaged Multifiber Composites, Sci. Eng. Comp. Mater. 7 (1-2) (1998) 204.

[392] L.S. Schadler and C. Galiotis, Fundamentals and Applications of Micro Raman Spectroscopy to Strain Measurements in Fibre Reinforced Composites, Inter. Mater. Rev. 40 (1995) 116.

[393] Ph. Colomban, Analysis of Strain and Stress in Ceramic, Polymer and Metal Matrix Composites by Raman Spectroscopy, Adv. Eng. Mater. 4 (2002) 535.

[394] B. Prinz and E. Schnack, Determination of Stress Gradients in Fibrous Composites by X-Ray Diffraction from Fillers, J. Comp. Mater. 31 (1997) 852.

[395] C. Genzel, A Study of X-Ray Residual Stress Gradient Analysis in Thin Layers with Strong Fibre Texture. Part 1 : Evaluation of the Stress Factors $F_{i j}$, phys. stat. sol. (a) 165 (1998) 347.

[396] C. Genzel and W. Reimers, A Study of X-Ray Residual Stress Gradient Analysis in Thin Layers with Strong Fibre Texture. Part 2 : Examples, phys. stat. sol. (a) 166 (1998) 751.

[397] H. Choo, P. Rangaswamy and M.A.M. Bourke, Evolution of Phase Fraction and Elastic Lattice Strains in a Ti-6Al-4V/SiC Composite during Heating : a Neutron Diffraction Study, Proc. "ICCE/6", Orlando, FL, U.S.A., 127 (1999).

[398] M. Dutta, G. Bruno, L. Edwards and M.E. Fitzpatrick, Internal Stress Changes Measured by Neutron Diffraction in a Metal Matrix Composite after Mechanical and Thermal Treatments, Proc. "ICCE/6", Orlando, FL, U.S.A., 195 (1999).

[399] M. Surgeon, E. Vanswijgenhoven, M. Wevers and O. Van-Der-Biest, Acoustic Emission During Tensile Testing of SiC-Fibre-Reinforced BMAS Glass-Ceramic Composites, Composites A28 (1997) 473.

[400] S.-C. Wooh, Potential Use of Ultrasonic Phased Arrays for Non Destructive Evaluation of Concrete and Composite Materials, Proc. "ICCE/6", Orlando, FL, U.S.A., B41 (1999).

[401] H.E. Gundtoft and K.K. Borum, Characterization of Composites by UltrasonicScanning Examples and Experience from Participation in European and National Projects, Proc. "ICCE/6", Orlando, FL, U.S.A., 273 (1999).

[402] B. Fiedler and K. Schulte, Photo-Elastic Analysis of Fibre-Reinforced Model Composite Materials, Comp. Sci. \& Technol. 57 (1997) 859.

[403] W.K. Binienda, Photoelastic Visualisation of the Fracture Events in a Model of Functionaly Graded Material, Proc. "ICCE/6", Orlando, FL, U.S.A., 65 (1999).

[404] A. Dalmaz, P. Reynaud, D. Rouby and G. Fantozzi, Damage Propagation in Carbon/Silicon Carbide Composites During Tensile Tests Under the SEM, J. Mater. Sci. 31 (1996) 4213.

[405] C. Briançon, P. Sigety and C. G'Sell, In Situ Study of Matrix Strain in Carbon/Resin Composite Materials, Comp. Sci. \& Technol. 56 (1996) 835.

[406] H.-A. Crostack, G. Fischer, E. Soppa, S. Schmauder and Y.-L. Liu, Localization of Strain in Metal-Matrix Composites, Proc. "ICCE/6", Orlando, FL, U.S.A., 223 (1999).

[407] B. Passilly and M. Parlier, Caractérisation Mécanique des Interfaces et des Fibres dans les Composites à Matrice Céramique, Rapport technique ONERA nº 67/3548 MY (1994). 
[408] M. Drissi-Habti and K. Nakano, The Modelling of Shear Stress Transfer in HiNicalon/ $\alpha-\mathrm{Si}_{3} \mathrm{~N}_{4}$ Ceramic Matrix Composites by the Use of Micro-Indentation Tests, Comp. Sci. \& Technol. 57 (1997) 1381.

[409] C.-H. Hsueh, Some Considerations of Evaluation of Interfacial Frictional Stress from the Indentation Technique for Fibre-Reinforced Ceramic Composites, J. Mater. Sci. Lett. 8 (1989) 739.

[410] D.B. Marshall and W.C. Oliver, An Indentation Method for Measuring Residual Stresses in Fibre-Reinforced Ceramics, Mater. Sci. Eng. A126 (1990) 95.

[411] T. Weihs and W.D. Nix, Experimental Examination of the Push-Down Technique for Measuring the Sliding Resistance of Silicon Carbide Fibres in a Ceramic Matrix, J. Am. Ceram. Soc. 74 (1991) 524.

[412] P.W.J. van den Heuvel, B. Hogeweg and T. Peijs, An Experimental and Numerical Investigation into the Single-Fibre Fragmentation Test : Stress Transfer by a Locally Yielding Matrix, Composites A28 (1997) 237.

[413] W. Wu, M. Desaeger, I. Verpoest and J. Varna, An Improved Analysis of the Stresses in a Single-Fibre Fragmentation Test : I. Two-Phase Model, Comp. Sci. \& Technol. 57 (1997) 809.

[414] R.J. Young, Evaluation of Composite Interfaces Using Raman Spectroscopy, Key Eng. Mat. 116-117 (1996) 173.

[415] J.R. Wood, Y. Huang, R.J. Young and G. Marom, Measurement of Thermal Strains During Compressive Fragmentation in Single-Fibre Composites by Raman Spectroscopy, Comp. Sci. \& Technol. 55 (1995) 223.

[416] D. Lévêque and M.H. Auvray, Study of Carbon-Fibre Strain in Model Composites by Raman Spectroscopy, Comp. Sci. \& Technol. 56 (1996) 749.

[417] N. Melanitis, C. Galiotis, P.L. Tetlow and C.K.L. Davies, Interfacial Shear Stress Distribution in Model Composites Part 2 : Fragmentation Studies on Carbon Fibre/Epoxy Systems, J. Comp. Mater. 26 (1992) 574.

[418] D.J. Bannister, M.C. Andrews, A.J. Cervenka and R.J. Young, Analysis of the SingleFibre Pull-Out Test by Means of Raman Spectroscopy : Part II. Michromechanics of Deformation For an Aramid/Epoxy System, Comp. Sci. \& Technol. 53 (1995) 411.

[419] B. Guichet, J.-C. Sangleboeuf, A. Vassel and T. Bretheau, A Study of the Micromechanical Push-Out Test : Response of an SCS-6 / Ti6242 Composite, Comp. Sci. \& Technol. 58 (1998) 665.

[420] H. Ledbetter, Relationship between Bulk-Modulus Temperature Dependence and Thermal Expansivity, phys. stat. sol. (b) 181 (1994) 81.

[421] J.P. Petitet, Influence de la Pression sur les Spectres Raman. Corrélation avec les Propriétés Intra et Intermoléculaires, Proc. " Ecole Thématique de Spectroscopie Raman en Chimie et Physique des Matériaux", Autrans, France, (1998).

[422] M.A. White, Thermal Properties of Solids : Etude in Three-Part Anharmony, Can. J. of Chem. 74 (1996) 1916.

[423] B. Schrader, General Survey of Vibrational Spectroscopy, In: Infrared and Raman Spectroscopy, Methods and Applications (Chap. 1). Ed. B. Schrader, VCH, Weinheim, 7 (1995).

[424] B.A. Weinstein and R. Zallen, Pressure Raman Effects in Covalent and Molecular Solids, In: Topics in Applied Physics, Light Scattering in Solids IV. Eds. M. Cardona and G.Güntherodt, Springer-Verlag, Heidelberg, Germany, 463 (1984).

[425] A. Jayaraman, Diamond Anvil Cell and High-Pressure Physical Investigations, Rev. Mod. Phys. 55 (1983) 65. 
[426] J.R. Ferraro, Vibrational Spectroscopy at High External Pressures, The Diamond Anvil Cell, Academic Press, New York (1984).

[427] D. Olego, M. Cardona and P. Vogl, Pressure Dependance of the Optical Phonons and Transverse Effective Charge in 3C-SiC, Phys. Rev. B25 (1982) 3878.

[428] J.F. Di Gregorio and T.E. Furtak, Analysis of Residual Stress in 6H-SiC Particles within $\mathrm{Al}_{2} \mathrm{O}_{3} / \mathrm{SiC}$ Composites Through Raman Spectroscopy, J. Am. Ceram. Soc. 75 (1992) 1854.

[429] J. Liu and Y.K. Vohra, Raman Modes of 6H Polytype of Silicon Carbide to Ultrahigh Pressures : A Comparison with Silicon and Diamond, Phys. Rev. Lett. 72 (26) (1994) 4105.

[430] T.L. Schindler and Y.K. Vohra, A Micro-Raman Investigation of High-Pressure Quenched Graphite, J. Phys.: Cond. Matter 7 (1995) L637.

[431] M. Hanfland, K. Syassen, S. Fahy, S.G. Louie and M.L. Cohen, Pressure Dependence of the First-Order Raman Mode in Diamond, Phys. Rev. B31 (10) (1985) 6896.

[432] S.-J. Jeon, D. Kim, S.K. Kim and I.C. Jeon, High-Pressure Raman Study of Fullerite $C_{60}$, J. Raman Spectrosc. 23 (1992) 311.

[433] S.H. Tolbert, A.P. Alivisatos, H.E. Lorenzana, M.B. Kruger and R. Jeanloz, Raman Studies on $C_{60}$ at High Pressures and Low Temperatures, Chem. Phys. Lett. $188(3,4)$ (1992) 163.

[434] I.V. Aleksandrov, A.F. Goncharov, E.V. Yakovenko and S.M. Stishov, High Pressure Study of Diamond, Graphite and Related Materials, In: High-Pressure Research : Application to Earth and Planetary Sciences. Eds. Y. Syono and M.H. Manghnani, Terra Scientific Publishing Company (Tokyo) - American Geophysical Union (Washington, D.C.), 409 (1992).

[435] E. Anastassakis, A. Pinczuk, E. Burnstein, F.H. Pollak and M. Cardona, Effect of Static Uniaxial Stress on the Raman Spectrum of Silicon, Solid State Comm. 8 (1970) 133.

[436] E. Anastassakis, Selection Rules of Raman Scattering by Optical Phonons in Strained Cubic Crystals, J. Appl. Phys. 82 (1997) 1582.

[437] S.H. Shin, F.H. Pollak and P.M. Raccah, Effects of Uniaxial Stress on the Raman Frequencies of $\mathrm{Ti}_{2} \mathrm{O}_{3}$ and $\mathrm{Al}_{2} \mathrm{O}_{3}$, Proc. "3 ${ }^{\text {rd }}$ Inter. Conf. on Light Scattering in Solids", Paris, France, 401 (1975).

[438] W. Jia and W.M. Yen, Raman Scattering From Sapphire Fibres, J. Raman Spectrosc. 20 (1989) 785.

[439] T. Englert, G. Abstreiter and J. Pontcharra, Determination of Existing Stress in Silicon Films on Sapphire Substrate using Raman Spectroscopy, Solid State Elec. 23 (1980) 31.

[440] H. Mukaida, H. Okumura, J.H. Lee, H. Daimon, E. Sakuma, S. Misawa, K. Endo and S. Yoshida, Raman Scattering of SiC : Estimation of the Internal Stress in 3C-SiC on Si., J. Appl. Phys. 62 (1987) 254.

[441] J.W. Ager III and M.D. Drory, Quantitative Measurement of Residual Biaxial Stress by Raman Spectroscopy in Diamond Grown on a Ti Alloy by Chemical Vapor Deposition., Phys. Rev. B48 (1993) 2601.

[442] Y.M. Cheong, H.L. Marcus and F. Adar, Raman Microprobe Measurements of Residual Strains at the Interfaces of Si on Quartz, J. Mater. Res. 2 (1987) 902.

[443] H. Mohrbacher, K.V. Acker, B. Blanpain, P.V. Houtte and J.-P. Celis, Comparative Measurement of Residual Stress in Diamond Coatings by Low-Incident-Beam-AngleDiffraction and Micro-Raman Spectroscopy, J. Mater. Res. 11 (1996) 1776.

[444] I. De Wolf, H.E. Maes and S.K. Jones, Stress Measurements in Silicon Devices through Raman Spectroscopy: Bridging the Gap between Theory and Experiment, J. Appl. Phys. 79 (1996) 7148. 
[445] S. Rohmfeld, M. Hundhausen, L. Ley, C.A. Zorman and M. Mehregany, Quantitative Evaluation of Biaxial Strain in Epitaxial 3C-SiC Layers on Si(100) Substrates by Raman Spectroscopy, J. Appl. Phys. 91 (2002) 1113.

[446] T.A. Michalske, D. Tallant and W.L. Smith, Raman Study of Silica Glass Under Tensile Stress, Phys. Chem. of Glasses 29 (1988) 150.

[447] Ph. Colomban, Protonic Defects and Crystallization of Sol-Gel (Si,Ge) Mullites and Alumina, Proc. " $7^{\text {th }}$ CIMTEC", Montecatini Terme, It., Elsevier Publisher B.V., Amsterdam, 599 (1991).

[448] R.J. Young, D. Lu, R.J. Day, W.F. Knoff and H.A. Davis, Relationship between Structure and Mechanical Properties for Aramid Fibres, J. Mater. Sci. 27 (1992) 5431.

[449] G. Chollon, R. Pailler and R. Naslain, Structure, Composition and Mechanical Behaviour at High Temperature of the Oxygen-Free Hi-Nicalon Fibre, Ceram. Trans. 58 (1995) 299.

[450] G. Chollon, R. Pailler, R. Naslain and P. Olry, Correlation Between Microstructure and Mechanical Behaviour at High Temperatures of a SiC Fibre with a Low Oxygen Content (Hi-Nicalon), J. Mater. Sci. 32 (1997) 1133.

[451] P.A. Tarantili, A.G. Andreopoulos and C. Galiotis, Real-Time Micro-Raman Measurements on Stressed Polyethylene Fibers. 1. Strain Rate Effects and Molecular Stress Redistribution, Macromolecules 31 (1998) 6964.

[452] N. Melanitis, P.L. Tetlow, C. Galiotis and S.B. Smith, Compressional Behaviour of Carbon Fibres, Part II : Modulus Softening, J. Mater. Sci. 29 (1994) 786.

[453] C. Filiou, C. Galiotis and D.N. Batchelder, Residual Stress Distribution in Carbon Fibre/Thermoplastic Matrix Pre-Impregnated Composite Tapes, Composites 23 (1992) 28.

[454] N. Melanitis and C. Galiotis, Compressional Behavior of Carbon Fibres. Part 1 : a Raman Spectroscopy Study, J. Mater. Sci. 25 (12) (1990) 5081.

[455] M.S. Amer and L.S. Schadler, Stress Concentration Phenomenon in Graphite/Epoxy Composites : Tension/Compression Effects, Comp. Sci. \& Technol. 57 (1997) 1129.

[456] R.J. Young and R.J. Day, Application of Raman Microscopy to the Analysis of High Modulus Polymer Fibres and Composites, Brit. Polymer J. 21 (1989) 17.

[457] P.W.J. van den Heuvel, T. Peijs and R.J. Young, Failure Phenomena in TwoDimensional Multi-Fibre Microcomposites. Part 2: A Raman Spectroscopic Study of the Influence of Inter-Fibre Spacing on Stress Concentrations, Comp. Sci. \& Technol. 57 (1997) 899.

[458] V. Chohan and C. Galiotis, Effects of Interface, Volume Fraction and Geometry on Stress Redistribution in Polymer Composites under Tension, Comp. Sci. \& Technol. 57 (1997) 1089.

[459] C. Galiotis, A. Paipetis and C. Marston, Unification of Fibre/Matrix Interfacial Measurements with Raman Microscopy, J. Raman Spectrosc. 30 (10) (1999) 899.

[460] A. Paipetis and C. Galiotis, A Study of the Stress-Transfer Characteristics in Model Composites as a Function of Material Processing, Fibre Sizing and Temperature of the Environment, Comp. Sci. \& Technol. 57 (1997) 827.

[461] C. Marston, B. Gabbitas, J. Adams, P. Marshall and C. Galiotis, Measurement of Stress Concentration around Fibre Breaks in Carbon-Fibre/Epoxy-Resin Composite Tows, Comp. Sci. \& Technol. 57 (1997) 913.

[462] P.W.J. van den Heuvel, T. Peijs and R.J. Young, Analysis of Stress Concentrations in Multi-Fibre Composites by Means of Raman Spectroscopy, J. Mater. Sci. Lett. 15 (1996) 1908. 
[463] M.S. Amer and L.S. Schadler, The Effect of Interphase Toughness on Fibre/Fibre Interaction in Graphite/Epoxy Composites : An Experimental and Modelling Study, J. Raman Spectrosc. 30 (10) (1999) 919.

[464] S. Narayanan and L.S. Schadler, Assessment of Strains along Fiber Surface Features in Graphite/Epoxy Composites Loaded in Compression, Comp. Sci. Technol. 59 (10) (1999) 1589.

[465] M.C. Andrews and R.J. Young, Analysis of the Deformation of Aramid Fibers and Composites Using Raman Spectroscopy, J. Raman Spectrosc. 24 (1993) 539.

[466] J.A. Bennett and R.J. Young, Micromechanical Aspects of Fibre/Crack Interactions in an Aramid/Epoxy Composite, Comp. Sci. \& Technol. 57 (1997) 945.

[467] C. Galiotis, I.M. Robinson, R.J. Young, B.J.E. Smith and D.N. Batchelder, Strain Dependence of the Raman Frequencies of a Kevlar 49 Fibre, Polymer Comm. 26 (1985) 354.

[468] S. van der Zwaag, M.G. Northolt, R.J. Young, I.M. Robinson, C. Galiotis and D.N. Batchelder, Chain Stretching in Aramid Fibres, Polymer Comm. 28 (1987) 276.

[469] H.D. Wagner, M.S. Amer and L.S. Schadler, Fibre Interactions in Two-Dimensional Composites by Micro-Raman Spectroscopy, J. Mater. Sci. 31 (1996) 1165.

[470] M. Kawagoe, S. Hashimoto, M. Nomiya, M. Morita, J. Qiu, W. Mizuno and H. Kitano, Effect of Water Absorption and Desorption on the Interfacial Degradation in a Model Composite of an Aramid Fibre and Unsaturated Polyester Evaluated by Raman and FT Infra-Red Microspectroscopy, J. Raman Spectrosc. 30 (1999) 913.

[471] P. Masse, J.P. Cavrot, C. Depecker, J. Laureyns and B. Escaig, Characterization by Raman Microspectrometry of Residual Compressive Stresses Induced by Resin Curing in Single Filament Composites Samples, Macromolecular Symposia 94 (1995) 249.

[472] C.A. Cooper, R.J. Young and M. Halsall, Investigation into the Deformation of Carbon Nanotubes and their Composites through the Use of Raman Spectroscopy, Composites A32 (2001) 401.

[473] J.Z. Wan, F.H. Pollak and B.E. Dorfman, Micro-Raman Study of Diamond-Like AtomicScale Composite Films Modified by Continuous Wave Laser Annealing, J. Appl. Phys. 81 (1997) 6407.

[474] X. Yang, D.J. Bannister and R.J. Young, Analysis of the Single-Fiber Pullout Test Using Raman Spectroscopy : Part III, Pullout of Nicalon Fibers from a Pyrex Matrix, J. Am. Ceram. Soc. 79 (1996) 1868.

[475] J. Wu and Ph. Colomban, Raman Spectroscopy Study on the Stress Distribution in the Continuous Fibre-Reinforced CMC, J. Raman Spectrosc. 28 (1997) 523.

[476] G. Gouadec, S. Karlin, J. Wu, M. Parlier and Ph. Colomban, Physical Chemistry and Mechanical Imaging of Ceramic-Fibre-Reinforced Ceramic- or Metal-Matrix Composites, Comp. Sci. \& Technol. 61 (2001) 383.

[477] X. Yang and R.J. Young, Model Ceramic Fibre-Reinforced Glass Composites : Residual Thermal Stresses, Composites 25 (1994) 488.

[478] X. Yang and R.J. Young, Fibre Deformation and Residual Strain in Silicon Carbide Fibre Reinforced Glass Composites, Brit. Ceram. Trans. 93 (1994) 1.

[479] X. Yang and R.J. Young, The Microstructure of a Nicalon/SiC Composite and Fibre Deformation in the Composite, J. Mater. Sci. 28 (1993) 2536.

[480] F. Bollet, C. Galiotis and M.J. Reece, Characterization of Strain Distribution in Fibres Bridging Ceramic Matrix Cracks by LRS, Proc. " $7^{\text {th }}$ Eur. Conf. on Comp. Mater." Woodhead Publishing Ltd., Cambridge, UK, 505 (1996). 
[481] G. Pezzotti, In situ Study of Fracture Mechanisms in Advanced Ceramics using Fluorescence and Raman Microprobe Spectroscopy, J. Raman Spectrosc. 30 (10) (1999) 867.

[482] G. Chollon and J. Takahashi, La Microscopie Raman Appliquée aux Composites Carbone/Carbone, Proc. "JNC11", Arcachon, France, Editions AMAC, Paris, 777 (1998).

[483] S. Karlin, PhD Thesis. Analyse Chimique et Mécanique ex situ et in situ, de Fibres et Composites Céramiques (CMC). Apport de la Microscopie Raman, University of Montpellier 2 - Sci. et Techn. du Languedoc (1996).

[484] M. Monthioux, O.P. Bahl, R.B. Mathur, T.L. Dhami, H.O. Dwivedi and S.P. Sharma, Controlling PAN-Based Carbon Fibre Texture via Surface Energetics, Carbon 38 (2000) 475.

[485] D.D. Edie, The Effect of Processing on the Structure and Properties of Carbon Fibers, Carbon 36 (1998) 345.

[486] Ph. Colomban, G. Gouadec, J. Mathez, J. Tschiember and P. Pérès, Raman Stress Measurement in Opaque Industrial C $C_{f}$ epoxy Composites Submitted to Tensile Strain, Composites Part A: Appl. Sci. Manuf. 37 (2006) 646.

[487] Y.G. Gogotsi, A. Kailer and K.G. Nickel, Phase Transformations in Materials Studied by Micro-Raman Spectroscopy of Indentations, Mater. Res. Innov. 1 (1997) 3.

[488] M.S. Amer, J. Busbee, S.R. Leclair, J.F. Maguire, J. Johns and A. Voevodin, Non Destructive, In-Situ Measurements of Diamond-Like Carbon Film Hardness Using Raman and Rayleigh Scattering, J. Raman Spectrosc. 30 (1999) 947.

[489] X.J. Ning and P. Pirouz, The Microstructure of SCS-6 SiC Fibre, J. Mater. Res. 6 (10) (1991) 2234.

[490] A.B. Mann, M. Balooch, J.H. Kinney and T.P. Weihs, Radial Variations in Modulus and Hardness in SCS-6 Silicon Carbide Fibers, J. Am. Ceram. Soc. 82 (1999) 111.

[491] R. El-Mallawany and A. Abd-El-Moneim, Comparison between the Elastic Moduli of Tellurite and Phosphate Glasses, phys. stat. sol. (a) 166 (1998) 829.

[492] R.J. Day, I.M. Robinson, M. Zakikhani and R.J. Young, Raman Spectroscopy of Stressed High Modulus Poly(p-phenylene benzobisthiazole) Fibres, Polymer 28 (1987) 1833.

[493] B.J. Kip, M.C.P. Van-Eijk and R.J. Meier, Molecular Deformation of High Modulus Polyethylene Fibers Studied by MRS, J. Polymer Sci.: Part B 29 (1991) 99.

[494] A. Marcellan, Ph. Colomban and A. Bunsell, (Nano)Structure, Skin/Core and Tension Behaviour of Polyamide Fibres, J. Raman Spectrosc. 35 (2004) 308.

[495] C. Hierold, From Micro- to Nanosystems: Mechanical Sensors go Nano, J. Micromech. Microeng. 14 (2004) S1.

[496] O. Lourie and H.D. Wagner, Evaluation of Young's Modulus of Carbon Nanotubes by Micro-Raman Spectroscopy, J. Mater. Res. 13 (1998) 2418.

[497] J.R. Wood, Q. Zhao, M.D. Frogley, E.R. Meurs, A.D. Prins, T. Peijs, D.J. Dunstan and H.D. Wagner, Carbon Nanotubes: From Molecular to Macroscopic Sensors, Phys. Rev. B62 (11) (2000) 7571.

[498] Q. Zhao, M.D. Frogley and H.D. Wagner, The Use of Carbon Nanotubes to Sense Matrix Stress around a Single Glass Fiber, Comp. Sci. Technol. 61 (2001) 2139. 